\title{
Reliability and Validity of a Multi-Sensor Armband in Estimating Resting and Exercise Energy Expenditure
}

\author{
Margaret L. Fruin
}

Thesis submitted to the Faculty of the Virginia Polytechnic Institute and State University in partial fulfillment of the requirements for the degree of

Master of Science

in

Human Nutrition, Foods, and Exercise

Janet Walberg Rankin, Ph.D., Committee Chair

William Herbert, Ph.D.

Janet Wojcik, Ph.D.

June 4, 2003

Blacksburg, Virginia

Keywords: SenseWear Armband, Physical activity, Caloric expenditure, Activity monitoring, Motion sensors

Copyright 2003, Margaret L. Fruin 


\title{
Reliability and Validity of a Multi-Sensor Armband in Estimating Resting and Exercise Energy Expenditure
}

\author{
Margaret L. Fruin
}

\begin{abstract}
(ABSTRACT)
This study examined the reliability and validity of the SenseWear Armband (SWA, BodyMedia, Inc.) during rest and exercise compared to indirect calorimetry (IC). Energy expenditure (EE) was assessed with SWA and IC in 13 males during two resting and one cycle ergometry (40 min at $60 \% \mathrm{VO}_{2 \text { peak }}$ ) sessions. In a second experiment, 20 adults walked on a treadmill for 30 min at 3 intensities while IC and SWA measured EE. At rest, no significant differences were found between EE measurements from the SWA (1.3 $\pm 0.1 \mathrm{kcal} / \mathrm{min})$ and IC $(1.3 \pm 0.1 \mathrm{kcal} / \mathrm{min})$, and the methods were significantly correlated $(\mathrm{r}=0.76)$. The SWA EE estimation was reliable when comparing the two resting visits $(r=0.93)$. For the ergometer protocol, no significant differences were found between the SWA and IC measurements of EE early, mid, or late in exercise or for the total bout, although the measurements were not correlated $(r=0.03-0.12)$. The SWA EE estimate of walking increased with treadmill speed but not with inclination. The SWA significantly overestimated the EE of walking with no grade $(27.4 \%$ for $3 \mathrm{mph} ; 12.6 \%$ for $4 \mathrm{mph})$ and significantly underestimated EE on the $5 \%$ grade $(21.9 \%)(\mathrm{p}<0.02)$. The SWA estimation of EE correlated with IC $(\mathrm{r}=0.47-0.69)$. The SWA provided valid and reliable estimates of EE at rest. The SWA provided similar mean estimates of EE as IC on the ergometer, however the individual error was large. The SWA overestimated the EE of flat walking and underestimated inclined walking EE.
\end{abstract}




\section{ACKNOWLEDGEMENTS}

I would like to begin by thanking my wonderful family for their endless encouragement. I could never have made it through my school career this successfully without the love and support of my parents. I have learned so much from them and realize everyday the numerous ways I take after them-I am so lucky. I also want to thank my sister, Libby, for her compassion and friendship; may our relationship continue to grow stronger.

Dr. Rankin has been a great advisor during my graduate work at Virginia Tech. I have learned so much about research and scientific writing from her and could not have completed my thesis this spring without her guidance, quick revisions, encouragement, and dedication to my efforts. I would also like to thank my other committee members, Dr. Herbert and Dr. Wojcik, for their advice and help with the research plan. Dr. Herbert and Larry Miller were also very helpful in my understanding and interpretation of Bland-Altman bias plots.

I must also give a big thanks to Max Shute for teaching me the ins-and-outs of the indirect calorimetry systems and incorporating my armband into his CLA study. I thoroughly enjoyed working with him every morning last spring and thought our strengths really complemented each other. I wish him the best of luck in beginning his career on the other side of the podium.

This project could not have been completed without the help of many others. Specifically, Janet Rinehart went out of her way to help Max and me each morning with blood draws whether we were in Wallace or War; her help and flexibility were greatly appreciated. Dr. Schulman was an incredible help with developing and implementing the statistical analyses I performed. I was amazed by his quick responses to all of my questions and his willingness to meet whenever I needed further help. His patience and kindness will be remembered. There were also many people at BodyMedia, Inc. who helped me throughout this entire project. Dan, Suresh, Scott, Ray, Dave, and others were very helpful and lots of fun. I enjoyed working with them. 
I would like to thank all of the subjects in this study. The young men that came in early in the morning were very dedicated, conscientious, and fun to work with, making my work with human subjects a positive experience. I also want to thank the group of subjects who came into the lab in the afternoons or on the weekends and helped mainly as a favor to me. This project wouldn't have been the same without you.

My great friends from Marshalltown, K-State, and Dallas have been very important fixtures in my life and have helped make me the person I am today. The friends I've made while I've been at Virginia Tech have made the last two years a great experience. Serah and Danylle came into my life at just the right time, and I thank them for their friendship and all of the laughs. I'm just sorry we didn't spend as much time together during the spring. I enjoyed the time Sean and I spent together our first year at Tech; I always learned something new from Sean (he never ceased to surprise me). Mary Dean, Jeannemarie, Abby, Jaya, and many others (too many to name them all) were very special friends to me. I wish them the best of luck and hope we keep in contact.

And last, but definitely not least, I have to thank Jeff. He was a very patient and understanding sounding board who offered so much more encouragement and support than I could have ever hoped. I am so thankful he came into my life and stayed by me when I moved half-way across the country. He provides much needed balance and joy to my structured life; I couldn't be this happy without him. 


\section{TABLE OF CONTENTS}

ACKNOWLEDGEMENTS iii

LIST OF TABLES viii

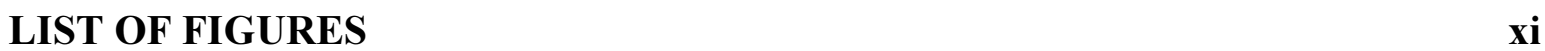

$\begin{array}{ll}\text { CHAPTER 1: Introduction } & 1\end{array}$

Introduction $\quad 2$

Statement of the Problem 3

Objectives 3

Hypotheses 3

Basic Assumptions $\quad 4$

Delimitations $\quad 4$

Limitations $\quad 5$

Definitions and Symbols $\quad 5$

CHAPTER 2: Review of Literature 6

Introduction $\quad 7$

Indirect Calorimetry and Doubly Labeled Water-the gold standards 8

Physical Activity Recall Questionnaires $\quad 9$

Physical Activity Records 14

$\begin{array}{ll}\text { Pedometers } & 17\end{array}$

$\begin{array}{ll}\text { Heart Rate Monitors } & 20\end{array}$

Uniaxial Accelerometers $\quad 24$

Triaxial Accelerometers 30

Use of Multiple Tools $\quad 42$

SenseWear ${ }^{\mathrm{TM}}$ Armband Description $\quad 44$ 
CHAPTER 3: Journal Manuscript $\quad 49$

$\begin{array}{lr}\text { Abstract } & 50\end{array}$

Introduction $\quad 51$

Methods $\quad 53$

Experiment 1 (EX1) 53

Experiment 2 (EX2) $\quad 54$

Data Analysis $\quad 55$

Statistical Analysis $\quad 55$

Results $\quad 56$

$\begin{array}{ll}\text { Discussion } & 58\end{array}$

References $\quad 65$

$\begin{array}{ll}\text { Tables } & 68\end{array}$

$\begin{array}{ll}\text { Figures } & 72\end{array}$

CHAPTER 4: Summary and Recommendations $\quad 80$

Recommendations for Future Research $\quad 83$

APPENDIX A: Detailed Description of Research Methods and Procedures 86

$\begin{array}{ll}\text { Experiment } 1 & 87\end{array}$

$\begin{array}{ll}\text { Subject Selection and Screening } & 87\end{array}$

$\begin{array}{ll}\text { Subject Pre-testing } & 87\end{array}$

Experimental Trials $\quad 88$

$\begin{array}{ll}\text { Resting Trials } & 88\end{array}$

Cycle Ergometry Trials $\quad 88$

$\begin{array}{lr}\text { Experiment } 2 & 89\end{array}$

$\begin{array}{ll}\text { Subject Selection and Screening } & 89\end{array}$

Experimental Trial $\quad 89$

Matching of EE Measurement Time Periods $\quad 90$

Indirect Calorimetry EE Determination $\quad 91$

SenseWear Armband EE Determination $\quad 92$ 


\section{LIST OF TABLES}

Table 1. Descriptive characteristics of subjects, Experiment 1.

Table 2. Descriptive characteristics of subjects, Experiment 2.

Table 3. Comparison of energy expenditure in $\mathrm{kcal} / \mathrm{min}$ for constant intensity cycle ergometer exercise by IC and SWA. Mean (SD).

Values are means of 3 (mid) or 5 (early and late) min measurement periods.

$\mathrm{IC}=$ indirect calorimetry

$\mathrm{SWA}=$ SenseWear Armband

Table 4. Comparison of energy expenditure in $\mathrm{kcal} / \mathrm{min}$ for walking intensities by the

SWA and IC. Mean (SD).

Values are means of 5 min measurement periods.

$\mathrm{IC}=$ indirect calorimetry

$\mathrm{SWA}=$ SenseWear Armband

${ }^{*} \mathrm{P}<0.05$ for comparison of devices

${ }^{* *} \mathrm{P}<0.01$ for comparison of devices

Table 5. Experiment 1 Individual Subject Demographics.

Table 6. Experiment 2 Individual Subject Demographics.

Table 7. Indirect Calorimetry and SWA Energy Expenditure Measurements (kcal/min): 96 CLA* Trial.

Values are means of 5 min measurement periods.

${ }^{*}$ CLA (conjugated linoleic acid) was treatment used for a companion study.

Table 8. Indirect Calorimetry and SWA Energy Expenditure Measurements (kcal/min): 97 Placebo* Trial.

Values are means of 5 min measurement periods.

*Placebo was olive oil treatment used for a companion study.

Table 9. Cycle Ergometer Exercise Indirect Calorimetry and SWA Energy Expenditure Measurements (kcal/min): CLA* Trial.

Values are means of 3 (mid) and 5 (early and late) min measurement periods.

${ }^{*}$ CLA (conjugated linoleic acid) was treatment used for a companion study.

This data was not included in analysis as work intensity was not held constant compared to the placebo trial.

Table 10. Cycle Ergometer Exercise Indirect Calorimetry and SWA Energy Expenditure Measurements ( $\mathrm{kcal} / \mathrm{min})$ : Placebo* Trial.

Values are means of 3 (mid) and 5 (early and late) min measurement periods.

*Placebo was olive oil treatment used for a companion study. 
Table 11. Cycle Ergometer Total 40 min Exercise Energy Expenditure Measurements (kcal) for Indirect Calorimetry and SWA: Placebo* Trial.

*Placebo was olive oil treatment used for a companion study.

Table 12. Cycle Ergometer Exercise Recovery Energy Expenditure (kcal) of min 4-8 for Indirect Calorimetry and SWA: Both CLA* Trial and Placebo ${ }^{* *}$ Trial.

${ }^{*}$ CLA (conjugated linoleic acid) was treatment used for a companion study.

**Placebo was olive oil treatment used for a companion study.

Table 13. Treadmill Exercise Indirect Calorimetry and SWA Energy Expenditure Measurements (kcal/min).

Values are means of 5 min measurement periods.

Table 14. Treadmill Exercise Recovery Energy Expenditure Measurements (kcal) of min 6-10 for Indirect Calorimetry and SWA.

Table 15. RM ANOVA for Resting EE Estimate by SWA

Note: The usual degrees of freedom were corrected by the Huynh-Feldt epsilon value.

Table 16. RM ANOVA for resting EE Measurement by IC.

Note: The usual degrees of freedom were corrected by the Huynh-Feldt epsilon value.

Table 17. Paired T-Test for Pooled Resting EE.

Table 18. Pearson's Correlation for Pooled Resting EE.

Table 19. Pearson's Correlation for SWA Resting EE—CLA Trial vs. Placebo Trial.

Table 20. Pearson's Correlation for IC Resting EE—CLA Trial vs. Placebo Trial.

Table 21. RM ANOVA for Ergometer Exercise EE.

Note: The usual degrees of freedom were corrected by the Huynh-Feldt epsilon value.

Table 22. RM ANOVA for Ergometer Exercise EE-Placebo Only.

Note: The usual degrees of freedom were corrected by the Huynh-Feldt epsilon value.

Table 23. Paired T-Test for SWA Estimate vs. IC Measurement of EE in Ergometer Exercise-Placebo Only.

Table 24. Pearson's Correlation for SWA Estimate vs. IC Measurement of EE in Ergometer Exercise-Placebo Only.

Table 25. Paired T-Test for SWA Estimate vs. IC Measurement of Relative EE in the Early Time Period Ergometer Exercise-Placebo Only. 
Table 26. Pearson's Correlation for SWA Estimate vs. IC Measurement of Relative EE 111 in the Early Time Period Ergometer Exercise-Placebo Only.

Table 27. Paired T-Test for Total Ergometer Exercise EE, Placebo Only.

Table 28. Pearson's Correlation for Total Ergometer Exercise EE-Placebo Only.

Table 29. RM ANOVA for Ergometer Exercise EE of Recovery min 4-8.

Table 30. Paired T-Test for Ergometer Exercise EE of Recovery min 4-8-Placebo Only.

Table 31. Pearson's Correlation for Ergometer Exercise EE of Recovery min 4-8Placebo Only.

Table 32. RM ANOVA for Treadmill Exercise EE.

Note: The usual degrees of freedom were corrected by the Huynh-Feldt epsilon value.

Table 33. Paired T-Test for SWA Estimate vs. IC Measurement of EE in Treadmill Exercise.

Table 34. Pearson's Correlation for SWA Estimate vs. IC Measurement of EE in Treadmill Exercise.

Table 35. Paired T-Test for SWA Estimate vs. IC Measurement of EE in Treadmill Exercise-Males Only.

Table 36. Pearson's Correlation Coefficient for SWA Estimate vs. IC Measurement of EE in Treadmill Exercise-Males Only.

Table 37. Paired T-Test for SWA Estimate vs. IC Measurement of EE in Treadmill Exercise-Females Only.

Table 38. Pearson's Correlation Coefficient for SWA Estimate vs. IC Measurement of EE in Treadmill Exercise-Females Only.

Table 39. Paired T-Test for Treadmill Exercise EE of Recovery min 6-10.

Table 40. Pearson's Correlation for Treadmill Exercise EE of Recovery min 6-10.

Table 41. Finding the number of observations (n) needed to have a power equal to 0.80 .118 Power determined using Pearson-Hartley Power Charts. ${ }^{56}$ 


\section{LIST OF FIGURES}

Figure 1. Bland-Altman bias plot for average resting energy expenditure.

95\% Limits of Agreement $=-0.17$ to 0.20

$95 \% \mathrm{CI}=-0.04$ to 0.07

Figure 2. Bland-Altman bias plot for SWA mean resting energy expenditure-trial 1

vs. 2.

95\% Limits of Agreement $=-0.07$ to 0.10

$95 \% \mathrm{CI}=-0.01$ to 0.04

Figure 3. Cycle ergometer mean energy expenditure measured by IC and SWAearly, mid, and late in constant intensity exercise.

Values are means $( \pm \mathrm{SE})$ of $3(\mathrm{mid})$ and 5 (early and late) min measurement periods.

$\mathrm{IC}=$ indirect calorimetry

SWA $=$ SenseWear Armband

Figure 4. Bland-Altman bias plot for early (A), mid (B), and late (C) cycle ergometer exercise energy expenditure.

A. $95 \%$ Limits of Agreement $=-3.79$ to 2.12

$95 \% \mathrm{CI}=-1.75$ to 0.08

B. $95 \%$ Limits of Agreement $=-3.26$ to 2.52

$95 \% \mathrm{CI}=-1.26$ to 0.52

C. $95 \%$ Limits of Agreement $=-3.68$ to 3.45

$95 \% \mathrm{CI}=-1.22$ to 0.98

Figure 5. Mean walking exercise energy expenditure estimates of the SWA compared to measurements by IC.

Values are means $( \pm \mathrm{SE})$ of 5 min measurement periods.

$\mathrm{IC}=$ indirect calorimetry

$\mathrm{SWA}=$ SenseWear Armband

Figure 6. Bland-Altman bias plot for $3 \mathrm{mph}, 0 \%$ grade walking energy expenditure.

$95 \%$ Limits of Agreement $=-0.29$ to 3.25

$95 \% \mathrm{CI}=1.06$ to 1.90

Figure 7. Bland-Altman bias plot for $4 \mathrm{mph}, 0 \%$ grade walking exercise energy

expenditure.

95\% Limits of Agreement $=-1.90$ to 3.62

$95 \% \mathrm{CI}=0.20$ to 1.52

Figure 8. Bland-Altman bias plot for $4 \mathrm{mph}, 5 \%$ grade walking exercise energy expenditure.

95\% Limits of Agreement $=-6.21$ to 2.52

$95 \% \mathrm{CI}=-2.89$ to -0.80 
Chapter 1

Introduction 
The importance of daily physical activity for health, particularly the prevention of certain chronic diseases, is widely reported by government agencies and health professionals. ${ }^{9,71}$ Obesity is one public health concern in the U.S. that can be positively influenced by physical activity. Approximately $62-67 \%$ of American adults are overweight (body mass index (BMI) $\geq 25 \mathrm{~kg} / \mathrm{m}^{2}$ ) and an estimated $30.5 \%$ are obese $\left(\mathrm{BMI} \geq 30 \mathrm{~kg} / \mathrm{m}^{2}\right){ }^{21}$ The obesity rates are particularly alarming because an association has been shown to exist between obesity and heart disease, diabetes, cancer, hyperlipidemia, and other health conditions. ${ }^{32,71}$ There is no doubt that physical activity can induce an energy deficit, which can lead to weight loss, if energy intake is stable. And it is widely believed that the modern inactive lifestyles of Americans are a large contributor to the widespread obesity epidemic, ${ }^{61}$ but many researchers have difficulty showing this in large population studies. This is primarily because determining the amount of physical activity performed by a population or individual is difficult. Besides the expensive and often inaccessible doubly labeled water technique, no assessment tool has currently been available that can provide valid information on the longterm levels of physical activity in populations. ${ }^{61}$ Physical activity records and recall questionnaires have been commonly used to approximate energy expenditure, while other energy expenditure measurement tools have been developed that are less dependent on the reporting accuracy of the subject. Pedometers, heart rate monitors, and uniaxial and triaxial accelerometers have all been used to quantify energy expenditure, although to varying degrees of accuracy. An accurate tool to measure energy expenditure outside of the laboratory is needed for a variety of uses: to accurately assess the physical activity of a population for epidemiological work, to effectively counsel and monitor clients in a weight loss or clinical setting, to help individuals gage their own activity on a daily basis, to justify and better define the metabolic equivalent (MET) levels of commonly performed activities, and to conduct research on physical activity and energy expenditure outside of the laboratory.

A new energy expenditure monitor, the SenseWear ${ }^{\mathrm{TM}}$ Armband (BodyMedia, Inc.), has recently become available that looks to overcome the problems of the other measures of freeliving energy expenditure, although no current studies have been published on the reliability or validity of this device. The validation of the SenseWear Armband in measuring energy expenditure at rest and exercise is needed to determine the effectiveness of this tool in assessing energy expenditure. 


\section{Statement of the Problem}

The SenseWear Armband is the latest energy expenditure assessment tool for use outside laboratory conditions. If proven to generate reliable and valid energy expenditure measurements, health professionals, individuals, and researchers will benefit greatly due to its variety of uses. Before the SenseWear Armband can be tested in free-living conditions and eventually utilized publicly, laboratory tests must be performed to determine its validity in rest and exercise bouts compared with indirect calorimetry measurements of energy expenditure.

\section{Objectives}

- To determine the validity of the SenseWear Armband in measuring resting energy expenditure.

- To examine the reliability of the SenseWear Armband in measuring resting energy expenditure on the same individual participating in the same protocol on different days.

- To determine the validity of the SenseWear Armband in measuring energy expenditure during steady-state exercise on a cycle ergometer.

- To determine the validity of the SenseWear Armband in measuring energy expenditure during steady-state walking on a treadmill at varying intensities.

\section{Hypotheses}

$\mathrm{Ho}_{1}$ : The SenseWear Armband estimation of resting energy expenditure will not differ from the indirect calorimetry measurement of resting energy expenditure.

$\mathrm{Ho}_{2}$ : There will be no differences between the SenseWear Armband estimations of resting energy expenditure on the same individual assessed on different days.

$\mathrm{Ho}_{3}$ : The SenseWear Armband estimation of energy expenditure during steady-state cycle ergometer exercise will not differ from the indirect calorimetry measurement of energy expenditure. 
$\mathrm{Ho}_{4}$ : The energy expenditure of treadmill walking at varying intensities estimated by the SenseWear Armband will not differ from the indirect calorimetry measurement of the same exercise.

\section{Basic Assumptions}

Experiment 1

- Subjects participated in $\leq 3$ days of structured cardiovascular exercise per week prior to and during the study.

- Maximal effort was exerted in the aerobic fitness $\left(\mathrm{VO}_{2 \text { peak }}\right)$ test.

- Subjects honestly reported their dietary intake for the 24-hours prior to their first experimental trial.

- Subjects consumed a similar diet 24 hours before each experimental trial.

- Subjects fasted and refrained from exercise during the 10-12 hours prior to the experimental trials.

Experiment 2

- Subjects fasted and refrained from exercise during the 3-hours prior to the experimental trial.

\section{Delimitations}

\section{Experiment 1}

- Subjects were aerobically untrained, healthy males between the ages of 18-25 yr.

- Subjects resided in or near Blacksburg, Virginia and were Virginia Tech students.

- Subjects consumed placebo (olive oil) or CLA capsules prior to resting and cycle ergometer trials, although only the placebo trial was used in the cycle ergometer analyses.

- Experimental trials were separated by at least 2 days.

- Cycling exercise was performed at $60 \%$ of the subject's $\mathrm{VO}_{2 \text { peak }}$.

Experiment 2

- Subjects were healthy men and women between the ages of 18-35 yr.

- Subjects resided in or near Blacksburg, Virginia and were Virginia Tech students. 


\section{Limitations}

- Results can only be generalized to individuals of similar gender, age, and fitness level.

- Subjects were free-living, therefore, neither self-reported dietary intake nor fasting practices could be verified.

- The SWA energy expenditure estimation and indirect calorimetry energy expenditure measurement of specific time periods could not be matched exactly but were within $30 \mathrm{sec}$.

- Differing clothing or upper arm composition (e.g., fat mass, muscle mass) may have impacted the SWA data collection for energy expenditure estimation.

\section{Definitions and Symbols}

EX1

EX2

IC

DLW

SWA

EE

RER

$\mathrm{VO}_{2}$

CLA

ANOVA

RM
Experiment 1; involved the resting and cycle ergometry protocols

Experiment 2; involving the treadmill protocol

Indirect Calorimetry

Doubly Labeled Water

SenseWear Armband

Energy Expenditure; reported as kcal or kcal/min

(Respiratory Exchange Ratio) ratio of carbon dioxide expired to oxygen consumed

volume of oxygen consumed

Conjugated Linoleic Acid; isomer of trans-10, cis-12 CLA

used in this study

Analysis of Variance

repeated measures 
Chapter 2

Review of Literature 


\section{Introduction}

Quantifying physical activity or energy expenditure is a current challenge for epidemiologists, clinicians, researchers, and the general public. For example, energy expenditure needs to be matched to energy intake for body weight maintenance - a current struggle in America, reflected by the rising rates of overweight and obesity. Accurately quantifying the physical activity and related energy expenditure of the population would help epidemiologists better determine where disparities are occurring in order to target interventions most effectively. Cardiac rehabilitation specialists need to know if the exercise protocols they prescribe are being followed accurately and regularly. Research is needed to clarify the physical activity levels needed to improve health and prevent disease. Currently different recommendations include the Centers for Disease Control and Prevention and American College of Sports Medicine recommendation of accumulating 30 minutes or more

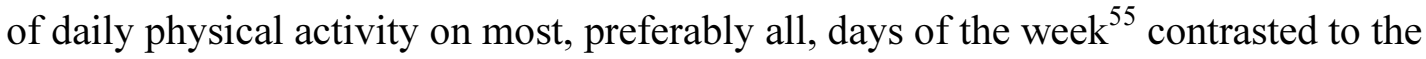
National Academy of Science Institute of Medicine recommendation of at least 60 minutes of daily physical activity to offset the increased caloric intake of Americans. ${ }^{49}$ On an individual level, quantifying the energy expenditure that results from varying activities and intensity levels would enhance weight loss goals and sport-specific training programs.

Energy expenditure can be accurately measured with indirect calorimetry in a laboratory/research setting and with doubly labeled water in a free-living environment (with some laboratory components). However, these techniques require expensive equipment and supplies and require knowledgeable technicians during the measurement period and subsequent analysis. Other tools have been developed to assess physical activity and energy expenditure outside of a laboratory setting for a lower cost. The accuracy of the energy expenditure estimation provided by these tools is determined by comparing the estimate to the measurement of indirect calorimetry or doubly labeled water-the gold standards in energy expenditure measurement.

Physical activity recall questionnaires and records are widely used in large and small studies because they are simple to use and low in cost. Recall questionnaires require the subject to remember the activity after it happens, while physical activity records usually require subjects to keep a log of their daily activities - physical and sedentary — as they are performed. Other tools have been developed to assess physical activity and/or energy 
expenditure objectively, such as pedometers, heart rate monitors, and accelerometers. Pedometers detect the up-and-down motion of the hip and record this motion as a step count which accumulates over time. Heart rate monitors detect and some types record the changing heart rate of individuals, which is often associated with energy expenditure. Also associated with energy expenditure is the acceleration of the body during movement, which accelerometers detect and record. Multiple tools have also been used together to assess physical activity and estimate energy expenditure. This chapter will review the literature regarding the validity and reliability of these tools in assessing physical activity and estimating energy expenditure.

The SenseWear ${ }^{\mathrm{TM}}$ Armband (BodyMedia, Inc., Pittsburgh, PA) is a physical activity and energy expenditure assessment tool that utilizes multiple sensors to collect a variety of physiologic data simultaneously. Proprietary algorithms use this data to generate information on physical activity patterns and to estimate energy expenditure. The reliability and validity of the SenseWear Armband's physical activity and energy expenditure estimations are not known, as no research has been published on this device.

\section{Indirect Calorimetry and Doubly Labeled Water-the gold standards}

Indirect calorimetry is one standard laboratory method commonly used to assess energy expenditure. The rates of oxygen consumed and carbon dioxide produced and the fuels being utilized are measured in a metabolic chamber over a period of time (e.g., 24 hours) or by using a mask, hood, or mouthpiece to analyze a specific period of time (e.g., exercise test). Total energy expenditure is determined using data on the amount of carbohydrate and fat utilized [respiratory exchange ratio (RER)] and the amount of oxygen consumed. ${ }^{47}$ This method has been validated as an accurate measure of energy expenditure, ${ }^{48}$ but it typically requires a laboratory setting or metabolic chamber. Portable indirect calorimetry systems have been developed and used in research to provide a valid energy expenditure measurement outside the laboratory; specifically the K4 Cosmed telemetric system (Roma, Italy), Cosmed K4 b $\mathrm{b}^{2}$, and Aerosport KB1-C (Ann Arbor, MI) portable indirect calorimeters have been shown to accurately measure energy expenditure over a wide range of exercise intensities. ${ }^{25,36,50}$ However, the use of portable units is limited due to the cost of the apparatus, the appearance and wearability of the device, and the technical knowledge required for its use. ${ }^{13}$ 
Doubly labeled water (DLW) is a valid technique for determining energy expenditure in free-living individuals. Doubly labeled water has been validated as a measure of energy expenditure in studies comparing the measurement to indirect or direct calorimeter chamber measurements or measurements where energy intake and utilization are determined in dietary balance studies. ${ }^{10}$ For example, Seale et al. ${ }^{62}$ found the average daily energy expenditure measurement made by DLW over 13 days did not differ from the measured metabolizable energy intake of men on a weight-maintenance diet. Doubly labeled water determines an individual's total energy expenditure. Indirect calorimetry is primarily used to determine resting metabolic rate (RMR); and thermic effect of food can be determined, but an estimate of $10 \%$ of total energy expenditure is commonly used. Daily physical activity energy expenditure can be determined from subtracting RMR and thermic effect of food from the total energy expenditure found from the DLW process. This process requires subjects to consume water labeled with ${ }^{2} \mathrm{H}\left({ }^{2} \mathrm{H}_{2} \mathrm{O}\right)$ and ${ }^{18} \mathrm{O}\left(\mathrm{H}_{2}{ }^{18} \mathrm{O}\right)$, and periodic urine samples are needed. The differential elimination rates of ${ }^{2} \mathrm{H}_{2}{ }^{18} \mathrm{O}$ versus $\mathrm{C}^{18} \mathrm{O}_{2}$ are determined which result in a measure of carbon dioxide production. ${ }^{47}$ Doubly labeled water is the gold standard for determining free-living energy expenditure over time (3 days to 3 weeks), but it does not provide information on the pattern or intensity of physical activity. This process is also limited because the labeled water and analysis process are very expensive and often hard to obtain.

Both indirect calorimetry and DLW provide the gold standards in energy expenditure measurement. They provide the energy expenditure measurements upon which all the other assessment tools are compared.

\section{Physical Activity Recall Questionnaires}

Of the different physical activity assessment methods available today, physical activity records and recall questionnaires provide the most cost effective option. There are instances, such as epidemiological or large studies, where a questionnaire is the only feasible way to assess physical activity. ${ }^{52}$ For this reason, it is important to continue to study and compare the strengths and weaknesses of the current questionnaires - including the method of administration, instructions provided to the subjects, validity and reliability of the estimation, and means of converting qualitative data into a quantified level of physical activity or estimation of energy expenditure. 
Physical activity recall questionnaires differ substantially, however, the overall principle remains the same- the subject relies on his or her memory to recall his or her activity after it happens. Physical activity recall questionnaires or surveys vary in terms of their complexity, time frame assessed, and types of activities surveyed. ${ }^{37}$ Surveys can be self-administered or interview-administered and range from single-item questions to more complex formats addressing a wide range of popular activities. ${ }^{37}$ Jacobs et al. ${ }^{31}$ found the accuracy of a questionnaire is not solely related to its length or complexity but to the logic used to construct the questions. Recall questionnaires are designed to estimate actual physical activity performed or usual/typical activity patterns. Questionnaires with short time frames (e.g., 24-hr, past week) often try to estimate actual activity, whereas longer time frames primarily reflect "usual" behavior (e.g., typical week, past year, lifetime). ${ }^{37}$ Logical questions must relate to either recent or habitual activity, as well as to the target population. Many physical activity questionnaires have been found to be most accurate in assessing high intensity activities, ${ }^{8,59}$ possibly due to the salient features of high intensity activity. ${ }^{14,59}$ This commonality among some of the general recall questionnaires results in estimates with less accuracy in women compared to men ${ }^{37,59}$ and older compared to younger subjects. ${ }^{14,64}$ Starling et al. ${ }^{64}$ reported that the age-neutral Minnesota Leisure Time Physical Activity Questionniare (LTA) was developed in men $\sim 40$ to $60 \mathrm{yr}$ of age and focused on higher intensity activities, which resulted in an underestimation of physical activity in older adults. The survey or questionnaire chosen for a study must elicit accurate information on the types of activity that contribute to the greatest amount of total energy expenditure in the population of interest. ${ }^{37}$ Currently many population (e.g., youth, elderly, women) or activity (e.g., sports, occupation, household chores) specific questionnaires are available with the intent to improve physical activity and energy expenditure estimation accuracy. The articles by Jacobs et al. ${ }^{31}$ and Bonnefoy et al. ${ }^{8}$ evaluate 10 physical activity questionnaires in adults and older men, respectively and may be helpful in comparing one to another. A detailed description of many commonly used questionnaires can be found in the June 1997 supplement of Medicine \& Science In Sports \& Exercise (volume 29, number 6).

Determining the validity of estimating physical activity energy expenditure with a record or recall questionnaire is best performed by comparing the estimation to a DLW measurement. The accuracy of two physical activity recall questionnaires was examined 
compared to DLW in older men $(n=32)$ and women $(n=35)$ in a study by Starling et al. ${ }^{64}$ The Minnesota Leisure Time Physical Activity Questionniare (LTA) assessed daily physical activity over the past 12 months, and the Yale Physical Activity Survey (YPAS) assessed a typical week of activity in the past month. The daily physical activity energy expenditure estimated by YPAS did not differ from that measured by DLW, however, the LTA estimate was significantly lower (by $\sim 50$ to $60 \%$, p $<0.05$ ) compared with DLW. These finding occurred in both men and women. The YPAS may have generated better results compared to the LTA because its assessment corresponded more directly to the DLW measurement time period. The YPAS compared favorably with measured mean physical activity energy expenditure on a group basis; however, the researchers found wide limits of agreement with this tool (-1310 to $1518 \mathrm{kcal} / \mathrm{d}$ for men, -963 to $981 \mathrm{kcal} / \mathrm{d}$ for women), making individual estimations poor. Physical activity recalls were also used by Leenders et al. ${ }^{38}$ who compared the 7-Day Physical Activity Recall (PAR) questionnaires of 13 women to DLW measurements. The physical activity energy expenditure group estimate for PAR $(642 \pm 35$ $\mathrm{kcal} / \mathrm{d})$ did not differ significantly from the DLW group estimate $(798 \pm 83 \mathrm{kcal} / \mathrm{d})$, whereas significant differences occurred consistently between the two measures in individuals (differences ranged from -633 to $280 \mathrm{kcal} / \mathrm{d}$ ) — both under- and overreporting occurred. Irwin et al. ${ }^{28}$ and Conway et al. ${ }^{17}$ found physical activity records to be more accurate than recall questionnaires. The researchers had 24 men extensively record all of their physical activity for seven days while undergoing DLW energy expenditure measurement. The Stanford Seven Day Recall Questionnaire (7DR) was administered by an interviewer at the end of the 14-day DLW measurement period. The 7DR has 14 items used to assess the time spent sleeping (1 MET) and in moderate, hard, and very hard physical activity (4, 6, and 10 METs, respectively). The remaining hours and minutes in the given day (24-hr period) represented the time spent in light intensity activities. The mean energy expenditure determined from physical activity records $(3385 \mathrm{kcal} / \mathrm{d})$ did not differ statistically from the mean DLW measurement $(3169 \mathrm{kcal} / \mathrm{d})$ - overestimation of $7.9 \pm 3.2 \%$. However, the 7DR significantly $(\mathrm{p}<0.05)$ overestimated the measured energy expenditure from DLW by $30.6 \pm$ $9.9 \%$ (means of $4157 \mathrm{kcal} / \mathrm{d}$ and $3169 \mathrm{kcal} / \mathrm{d}$, respectively). Neither the physical activity record nor 7DR estimation correlated with DLW $\left(\mathrm{R}^{2}=0.10\right.$ and 0.14 , respectively), representing the large individual error. 
In reviewing studies comparing activity questionnaires (both records and recalls) to DLW measurements, Westerterp ${ }^{78}$ also reported that activity records were superior to activity recall. As a result, it is not uncommon for researchers to use physical activity records as a criterion measure to evaluate physical activity recall questionnaire accuracy. Chasan-Taber et al. ${ }^{14}$ mailed a modified version of the Historical Leisure Activity Questionnaire to female college alumnae (131 women responded and completed the study). The self-administered questionnaire collected information on current activity over the past year. During the year prior to the completion of the questionnaire, the women completed two to four weekly physical activity logs (ideally one for each season), to serve as the criterion measure of physical activity. The logs divided activity into 15 -min intervals and were coded by the subject based on activity intensity. The total activity median from the questionnaire (58.2 MET-h/week) agreed relatively well with the median from the average of the logs (52.0 MET-h/week). The agreement was also good between the questionnaire and logs for moderate (39.2 MET-h/week vs. 39.7 MET-h/week, respectively) and vigorous (8.4 METh/week vs. 9.8 MET-h/week, respectively) activity. Unfortunately no means were provided for statistical comparison. The Pearson correlation coefficients between the two methods were modest for total activity $(\mathrm{r}=0.26)$, weak for moderate intensity activity $(\mathrm{r}=0.15)$, and highest for vigorous intensity activity $(\mathrm{r}=0.52)$. Given that physical activity logs are not a gold standard in assessing activity levels and have many sources of error, it is difficult to make conclusions regarding the validity of the questionnaire studied.

Determining the reliability of a physical activity recall questionnaire is often difficult because the correlation will not only reflect the measurement error but the variation in physical activity as well. ${ }^{60}$ Duncan et al. ${ }^{19}$ attempted to control for some of the physical activity variation impact when they examined the reliability of the PAR in estimating energy expenditure $(\mathrm{kcal} / \mathrm{kg} / \mathrm{d})$ over two consecutive weeks. The intraclass correlation coefficient was significant $(\mathrm{p}<0.05)$ among individuals who reported their activity as "typical" over both weeks assessed $(r=0.80,95 \% \mathrm{CI}=0.68-0.87)(\mathrm{n}=63)$, however, the relationship was lower in individuals who reported that at least one week was "not typical" of their usual physical activity pattern $(\mathrm{r}=0.44,95 \% \mathrm{CI}=0.26-0.59)(\mathrm{n}=94)$. Richardson et al. ${ }^{59}$ did not control for variations in physical activity assessed by two Stanford 7-Day Recall questionnaires administered 26 days apart. However, the results demonstrated modest but significant 
correlations between the two recalls (total activity: $r=0.60$ for men, $\mathrm{p}<0.01$ and $\mathrm{r}=0.36$ for women, $\mathrm{p}<0.05)$. Although the associations were significant, the researchers reported that their subjects had engaged in intensive activity monitoring for a year's duration and were highly motivated, making them not representative of the general adult population. In reviewing seven studies assessing the use of different physical activity recall questionnaires in adults, Sallis and Saelens ${ }^{60}$ reported the measures (e.g., Minnesota LTA, Seven-Day PAR) had relatively high test-retest reliabilities.

As previous findings demonstrate, physical activity recall questionnaires have the potential to adequately estimate free-living energy expenditure or physical activity patterns on a group or population basis but rarely demonstrate valid or reliable estimates for individuals. These findings should be expected due to the scoring or quantification method used. The vast majority of the researched physical activity questionnaires were quantified using the Compendium of Physical Activities. The first Compendium was published in $1993^{2}$ and the most recent version was published in $2000^{3}$. The 2000 Compendium breaks up physical activities into 21 major headings, giving each specific activity at five-digit code and intensity level based on the rate of energy expenditure-expressed as metabolic equivalents $(\mathrm{MET}){ }^{3}$ Metabolic equivalents are multiples of resting energy expenditure where $1 \mathrm{MET}$ represents $3.5 \mathrm{ml}$ of $\mathrm{O}_{2}$ per kg body weight per minute. ${ }^{10}$ Research has shown that absolute MET intensities provided in the Compendium of Physical Activities can be inaccurate for people of differing body weight and body fat composition. ${ }^{3,12}$ For example, heavier individuals expend more energy during weight-bearing activities than indicated by the MET intensities of the Compendium. It is speculated that inaccuracies also exist for individuals who differ in age, cardiorespiratory fitness, and mechanical efficiency and when geographic and environmental conditions vary. ${ }^{72}$ The Compendium of Physical Activities is limited in estimating the energy cost of physical activity in individuals, however, it was not developed for this purpose. ${ }^{3}$ The Compendium was designed to standardize the MET intensities of physical activity to classify activity for survey research and to enhance the comparability of results across studies using physical activity self-reports. ${ }^{3}$

The use of the Compendium of Physical Activities to score self-reported physical activity is common and widely accepted; however, different protocols are used and may impact the validity and reliability of the results. Some researchers use the specific codes from the 
Compendium that correspond to the actual activity recalled or recorded. This process is more time and labor intensive but could theoretically provide a better energy expenditure estimate. A more common, simplified method involves grouping activities into an intensity level category (e.g., light, moderate, hard, very hard) and then multiplying the time spent in each category by an average of MET intensity (e.g., moderate $=4.0$ METs). Given that the Compendium MET values are estimates themselves, the two scoring methods may produce similar results, although no studies have been published on this comparison.

Physical activity recall questionnaires are easy to administer and score, require very little subject effort or motivation, and are low in cost. ${ }^{10}$ These advantages make them the most commonly used physical activity assessment tools for epidemiological research. ${ }^{10}$ However, the validity and reliability of physical activity assessment with recall questionnaires is greatly influenced by the tool used, the method of administration, the time frame assessed, and the subjects studied. Over- and underestimation of the actual time and/or intensity of physical activity is common. ${ }^{78}$ Physical activity questionnaires are appropriate for and have the potential to accurately assess the physical activity of large groups or populations, however, their assessment of individual physical activity is limited.

\section{Physical Activity Records}

Physical activity records (also known as logs or diaries) are similar to physical activity recall questionnaires in terms of general convenience and low cost. The limitations between the two types of self-reports are also similar-differences in tools used make comparisons problematic; reliance on subject compliance and subjective interpretation of intensity affect validity and reliability; and quantification methods limit interpretations to group estimates. Making comparisons between different studies involving self-reported physical activity is often difficult due to differences in the tools used and the instructions given. Log books or sheets are often used when requiring subjects to keep physical activity records, to help standardize the recording process. The recording procedures tend to vary greatly from one study to another and should be considered when evaluating a study's results. For example, Irwin et al. ${ }^{28}$ and Conway et al. ${ }^{17}$ had their subjects use a very comprehensive recording style. The researchers had their subjects make a new entry in the provided record book each time a change in activity occurred and provide a detailed description for each entry/activity-1) time began, 2) body position, 3) description of activity, 4) perceived effort, and 5) purpose 
from a list of activity groups. A similar detailed description of activity was required by Richardson et al., ${ }^{59}$ however, the recording booklet used was divided into four hour segments. The subjects did not make a new entry for every activity but recorded the activities that occurred over the four hour time period. This type of recording would help reduce subject burden, however, some accuracy may be lost initially. However, if the recording duration is long, the increased detail may cause subjects to be less conscientious and therefore less accurate. Another common method involves recording activity in 15-min intervals with less extensive description and entry detail. ${ }^{14,41}$

Self-report records typically benefit from the conscientiousness of subjects as they record their activity as it happens or at least regularly throughout the day. However, a few studies relied on the subject to recall their activity at the end of the day ${ }^{1}$ or after segments (i.e., morning, afternoon, evening) of the day. ${ }^{19}$ These studies used a form or log that listed activities - very similar to a recall questionnaire form. This method was more similar to a 24-hr recall questionnaire than an activity record or diary in terms of strengths and weaknesses. The differences in physical activity records and instructions given make comparisons between some studies difficult.

As with recall questionnaires, DLW (or indirect calorimetry in a room calorimeter) is the best method to validate physical activity records. Litchman et al. ${ }^{41}$ compared 14 days of selfreported physical activity to DLW measurements in obese men and women. The physical activity records involved recording activity in 15 -min intervals throughout the day. The activity was quantified by grouping the activity into 10 categories and multiplying the time spent in each category by the corresponding energy expenditure estimate. Overestimation of self-reported physical activity approximated 30 to $50 \%$ in the subjects compared to the physical activity energy expenditure of the DLW measurement. Buchowski et al. ${ }^{12}$ also found low physical activity record validity when comparing self-reported activity diaries to energy expenditure measured in a room calorimeter (over two separate 24-hr periods). The subjects recorded the type, intensity, and start and end time of their activity at least every hour. The individual physical activity recording errors ranged from -25 to $42 \%$ in the 115 adults studied. More positive findings resulted when Bratteby et al. ${ }^{11}$ compared self-reported physical activity diaries to DLW in 15 year old adolescents $(n=50)$. The 25 male and 25 female subjects kept activity diaries (AD) for seven days during a 14-day DLW 
measurement. These diaries contained 15-min recording intervals, however, the subject entered a categorical value (number ranging from 1-9 representing the intensity of the dominant activity of that period) from a provided table instead of the activity performed. Although the subjects scored their own activity, the researchers provided detailed instructions and discussed the records with the subjects after the seven day recording period. The difference in total energy expenditure measured by DLW and estimated with the AD was not significant $-1.2 \%$ mean difference $(0.6 \%$ in males, $3.5 \%$ in females $)$. The researchers did find wide limits of agreement between the methods (no values were reported), implying AD provide a suitable estimate in groups but not individuals.

Physical activity records are often compared against other objective measures of physical activity, such as pedometers, accelerometers, and HR monitors. These objective measures cannot accurately quantify absolute energy expenditure (discussed in following sections), however, they can be used to identify activity periods and patterns. Jakicic et al. ${ }^{33}$ used the Tri-Trac accelerometer (Hemokinetics, Madison, WI) to verify self-reported exercise in 50 overweight women over six days. The women were considered active when the Tri-Trac registered an energy expenditure of $\geq 3 \mathrm{kcal} / \mathrm{min}$. The bouts of exercise were compared to the self-reported records (only the actual time spent exercising was recorded) and verified if a match occurred within 60 minutes. Jakicic et al. ${ }^{33}$ found that approximately 40 to $60 \%$ of the women over-reported their amount of exercise or exercise time. Those women who overreported their amount of exercise lost significantly less weight $(6.3 \pm 3.6 \mathrm{~kg})$ over the 20 week program than women who under/accurately reported their exercise $(9.4 \pm 5.2 \mathrm{~kg})$ $(p<0.02)$. A similar trend occurred for women who over-reported the amount of time they exercised, however, the difference was not significant $(\mathrm{p}<0.07)$. A HR monitor was used by Duncan et al. ${ }^{19}$ to assess the time and intensity of self-reported physical activity in sedentary men and women $(n=66)$. The interviewer-administered 7-day Physical Activity Recall (PAR) was used to assess activity over the past seven days; one of those days was assessed with a downloadable HR monitor and a self-report physical activity log (LOG). The LOG was a one-day replication of the PAR data form. The PAR and LOG were quantified by multiplying the time spent in each activity category by an estimated MET value (sleep $=1$ MET to very hard = $10 \mathrm{METs}$ ). The HR data was segmented into activity intervals lasting at least $10 \mathrm{~min}$ in length, and each activity interval was compared directly to an activity 
category based on each subject's individual heart rate reserve. These procedures put the PAR, LOG, and HR data into comparable MET values. Duncan et al. ${ }^{19}$ found that the sedentary subjects significantly $(\mathrm{p}<0.001)$ overestimated the amount of moderate intensity activity they performed with the PAR and LOG compared to that measured by HR. The hard intensity activity was also overestimated by LOG $(\mathrm{p}<0.02)$ but not by PAR. The three measures did not differ in assessing very hard activity. It is important to note that very few individuals engaged in hard or very hard activity.

Current research has shown that certain populations differ in their physical activity reporting accuracy. Individuals with a higher body fat percentage have been shown to significantly overestimate physical activity energy expenditure on records to a greater degree than leaner individuals. ${ }^{12,28}$ The recording accuracy of individuals has also been shown to increase as level of physical activity increased. ${ }^{28}$ The findings are of great concern as the majority of adults in the U.S. are overweight and sedentary.

Researchers using physical activity records have similar problems in determining the reliability of the method as those using physical activity recall questionnaires. This is due to not being able to distinguish between measurement error and variation in physical activity over the time periods assessed. ${ }^{60}$ The quantification of physical activity on records is also primarily based on the Compendium of Physical Activities, resulting in the same limitations mentioned in the previous section.

Physical activity records are advantageous due to their low cost, relatively simple quantification, and improved accuracy over physical activity recall questionnaires; although, much effort is required by study subjects and many sources of error exist. With proper instruction, adequate record detail, and sufficient subject compliance, physical activity records can provide highly accurate estimates of physical activity energy expenditure for groups. However, as with physical activity recall questionnaires, the accuracy of individual estimates is variable and typically low. ${ }^{10}$

\section{Pedometers}

Pedometers provide an assessment of physical activity based on the number of steps taken over a period of time, often per day. Modern pedometers are electronic and contain a horizontal, spring-suspended lever arm that deflects with the up-and-down motion or vertical acceleration of the hip. ${ }^{70}$ When the vertical acceleration exceeds a specific threshold value 
(e.g., force $\geq 0.35 \mathrm{x} \mathrm{g}$ ) an electrical circuit opens and closes and a step is recorded. ${ }^{69,77}$ The detection of vertical acceleration makes pedometers most sensitive to ambulatory activitieswalking and running.

The criterion standard for measuring steps taken is observation, although observation is only feasible in a laboratory or controlled environment. ${ }^{70}$ Welk et al. ${ }^{77}$ examined the reliability and validity of the Yamax Digi-Walker pedometer (Yamax Inc., Tokyo, Japan) for recording steps in a small pilot test. Thirty-one adults (17 men, 14 women) walked or jogged down an indoor hallway wearing the Digi-Walker while two observers recorded their step counts. The mean measured step counts were within $3-5 \%$ of the observer recorded values. For individuals, the Digi-Walker step count was within $10 \%$ for $81 \%$ of the walking trials (26 of 31 ) and $90 \%$ of the jogging trials (28 of 31 ). The intraclass reliability coefficient was moderate for walking $(\mathrm{R}=0.56)$ and high for jogging $(\mathrm{R}=0.89)$. In reviewing studies looking at the relationship between pedometers and observation, Tudor-Locke et al. ${ }^{70}$ found time in observed activity correlated positively with pedometer step counts (median of $\mathrm{r}=$ 0.82 , range $=0.42$ to 0.97$)$. The more ambulatory the physical activity assessed, the stronger the relationship.

Hendelman et al. ${ }^{27}$ found a similarly high correlation $(\mathrm{r}=0.84)$ between the Yamax Digiwalker pedometer (model SW-701) output and measured step frequency when 25 adults walked at four self-selected speeds on an indoor track. However, the Digiwalker pedometer significantly underestimated $(\mathrm{p}<0.001)$ the number of steps taken per minute by subjects at lower self-selected speeds ("leisurely" and "comfortable") compared with the measured step frequency, although the values became more similar for the faster walking bouts. At the fastest speed ("brisk") the Digiwalker did not differ significantly $(p>0.08)$ from the measured step frequency. Evidence from other investigations have been consistent with these results-pedometers have reduced accuracy during slow walking, possibly due to the generation of vertical acceleration forces less than the instrument sensitivity threshold. ${ }^{70}$

Accelerometers (to be discussed later) and pedometers are both objective motion sensors sensitive to vertical accelerations. ${ }^{70}$ Accelerometers are superior to pedometers in terms of technological ability and capacity, however their cost (as much as $\$ 450$ per unit) may make them a less feasible tool. ${ }^{69}$ Pedometers have been compared to accelerometers in studies to determine if the lower-tech, less expensive tool (\$10-50 per unit) generates comparable 
measurements. ${ }^{69}$ In a study by Tudor-Locke et al. ${ }^{69} 52$ adult volunteers wore the CSA accelerometer (model 7164, Version 2.2) and Yamax pedometer (PED; model SW-200) simultaneously during all waking hours for seven days. The mean PED-steps/d correlated with the CSA-steps $/ \mathrm{d}(\mathrm{r}=0.86 ; \mathrm{P}<0.0001)$. However, the recorded CSA-steps/d $(11,483 \pm$ $3856)$ were significantly greater than the PED-steps/d $(9638 \pm 4030)(\mathrm{P}<0.0001)$-mean difference approximately 2000 steps/d. The researchers stated that the large difference in recorded steps per day was likely due to the differences in force sensitivity to detect vertical accelerations $(\geq 0.30 \mathrm{x}$ g for CSA, $\geq 0.35 \mathrm{xg}$ for PED). A review of research comparing pedometers and accelerometers found a similarly high correlation (median $\mathrm{r}=0.86$, range $=$ nonsignificant to 0.99 ); the most highly representative output was found between pedometers and uniaxial accelerometers. ${ }^{70}$ Due to differences in technology and sensitivity, pedometers and uniaxial accelerometers do not appear to be easily substituted for one another; however, they appear to provide similar relative values and could both be used to assess primarily ambulatory physical activity. ${ }^{69}$

Some pedometers have become more than step counters and have the capability to estimate energy expenditure. Caloric expenditure is estimated by the manufacturer's proprietary regression equation using the user's body weight and step counts. ${ }^{39}$ The estimates provided by these pedometers have been compared to energy expenditure measurements of indirect calorimetry and DLW. Strath et al. ${ }^{65}$ compared the energy expenditure estimates provided by the Yamax SW-701 pedometer to the energy expenditure values measured by a portable indirect calorimetry unit (Cosmed K4b², S.r.L, Rome, Italy). The Yamax pedometer estimates caloric expenditure after the participant's body weight and stride length are entered; for this study, an assumed stride length of 2.5 feet was used. Thirty participants wearing the Yamax pedometer and Cosmed $\mathrm{K} 4 \mathrm{~b}^{2}$ engaged in a range of activities, each lasting 15 minutes preceded by 5 minutes of rest. The activity categories included housework $(n=11)$, yardwork $(n=9)$, and conditioning $(n=10)$. All energy expenditure data was converted into METs. The relationship between the MET values predicted by the Yamax to the measured METs was $\mathrm{R}^{2}=0.36$. The Yamax pedometer significantly underestimated the measured METs by $59.2 \%$ (average of 1.2 METs, $\mathrm{p}<0.001$ ). The input of each individual's actual stride length into the Yamax pedometer may have improved its estimate, although the improvement would have been slight primarily due to the 
limited ambulation in many lifestyle activities (e.g., washing dishes, gardening). In a study by Leenders et al., ${ }^{38}$ the Yamax-Digiwalker-500 was worn by 13 women for 7 days while undergoing DLW measurement. This particular pedometer estimated caloric expenditure based on steps taken and body mass using a proprietary equation. Daily physical activity energy expenditure approximated by the Yamax-Digiwalker-500 was underestimated by 59\% when compared to physical activity energy expenditure determined by DLW. The energy expenditure estimations of more advanced pedometers have been shown to be poor, so caution should be exercised if using them in this manner.

The low cost of most pedometers is one advantage for use by individuals desiring to increase their physical activity by daily walking. Recently pedometers (using the raw step count) have become a popular physical activity assessment tool and behavior change motivator for individuals whose primary physical activity is walking, however, their usefulness in other areas is minimal. Pedometers are limited in their assessment of physical activity because they cannot capture the pattern, intensity, or type of physical activity. ${ }^{70}$ However, when a raw indication of movement is needed, pedometers provide a cost-efficient option over uniaxial accelerometers. ${ }^{70}$ According to Leenders et al. ${ }^{38}$ a stepcounter may be useful in large studies that want to rank individuals by level of physical activity but cannot be used to determine physical activity energy expenditure.

\section{Heart Rate Monitors}

Heart rate monitoring is another objective method of physical activity assessment. Heart rate is a physiological parameter positively associated with energy expenditure during large muscle dynamic exercise. ${ }^{24,67}$ Relatively inexpensive heart rate monitors have become popular among individuals and clinicians for assessing exercise intensity. Research has benefited from advancements in technology which have led to the development of heart rate recorders that are able to store data over periods of days or weeks, thus providing information on the pattern of physical activity. ${ }^{67}$

Heart rate corresponds linearly with $\mathrm{VO}_{2}$ (energy expenditure) in higher levels of activity, making heart rate monitors useful tools in estimating energy expenditure during moderate to vigorous dynamic exercise. But at low activity levels, the relationship between heart rate and

energy expenditure is weak. ${ }^{51,76}$ Meijer et al. ${ }^{51}$ compared the monitored heart rate of 16 subjects performing a range of activities, each for 5 minutes, to energy expenditure measured 
by indirect calorimetry. Simple linear regression was used to calculate individual regression lines representing the relationship between heart rate and energy expenditure. The researchers found that the relationship between heart rate and energy expenditure was highly linear at high exercise intensities but deviated largely from linearity in lower intensity activity (no numerical values were reported). This research demonstrates the weak relationship between heart rate and energy expenditure at low activity intensity. In another study Welk et al. ${ }^{76}$ assessed the activities of young children with a heart rate monitor compared to a direct observation measure of physical activity. The direct observation method used was the Children's Activity Record System (CARS) which uses five different activity categories $(1=$ stationary to $5=$ fast translocation $)$ to classify physical activity. Minute-by-minute activity was assessed with the CARS and heart rate monitor (average of each minute compared) during a 40-min classroom period and a 30-min physical education (PE) period. The mean correlations between the heart rate and CARS scores for the 26 participants were higher during PE activities $(r=0.79)$ than classroom activities $(r=0.49)$. These results also demonstrate that the heart rate monitor provides a better measure of activity during active rather than inactive periods.

Luke et al. ${ }^{46}$ found a stronger association between heart rate and energy expenditure during low intensity activity for a group, but individual error was high. The researchers compared the energy expenditure assessment using heart rate to indirect calorimetry with a portable metabolic cart (MMC Horizon, Sensor Medics) in 10 subjects performing two tests: the Activities of Daily Living Circuit (ADLC) and a submaximal treadmill test. The ADLC consisted of three stages (sitting at rest, shopping for groceries, and vacuuming) for 3 minutes each. The treadmill test consisted of the following seven 3-min stages: stages 1-4 involved walking on a $3 \%$ incline while speed increased from 2.4 to $3.6 \mathrm{mph}$ and stages 5-7 involved walking at $3 \mathrm{mph}$ with inclinations of 6,9 , and $12 \%$. Individual general linear models were created in order to predict energy expenditure from heart rate data. Heart rate was shown to be a good predictor of energy expenditure on the treadmill test (mean $\mathrm{r}^{2}=$ $0.90)$, and the correlation coefficients for the ADLC were quite high as well (mean $\mathrm{r}^{2}=0.81$ ). During the ADLC the $\mathrm{VO}_{2}$ predicted by heart rate was greater than the measured $\mathrm{VO}_{2}$ by 4.5 $\pm 8.3 \%$ (individual range of -16 to $12 \%$ ); and during the treadmill test the prediction improved, differing by only $1.0 \pm 3.7 \%$ (individual range of -5 to $7 \%$ ). The heart rate 
prediction of energy expenditure was better in this study than the previous two, possibly because the intensity of the exercises was higher, eliciting a higher heart rate.

The accuracy of heart rate monitoring as a measure of energy expenditure has other limitations as well as being a poor energy expenditure predictor at low activity levels. The slope of the relationship between heart rate (HR) and oxygen consumption $\left(\mathrm{VO}_{2}\right)$ varies between individuals depending on their age and level of fitness, causing the need for individual calibration curves. ${ }^{24,45}$ Individualized $\mathrm{HR}-\mathrm{VO}_{2}$ calibration curves are generated, following laboratory exercise and rest measurements, to convert measured heart rate values into $\mathrm{VO}_{2}$ or energy expenditure predictions. ${ }^{10}$ Unfortunately, as seen in the studies mentioned previously, the generation of a single calibration curve does not often produce sufficient predictions. The slope of the relationship differs during exercise depending on whether the exercise involves the arms or the legs and how much of the activity is static or heavy resistance versus dynamic or low resistance. ${ }^{24}$ External factors such as emotion, heat, and posture also influence heart rate, which negatively impacts the use of heart rate as an exclusive measure of energy expenditure in normal life. ${ }^{24,51}$

More complex heart rate (HR) prediction methods have been developed to improve the use of heart rate for estimating energy expenditure. The Flex HR method (or HRFlex) has been used in studies to discriminate between rest and physical activity ${ }^{20}$ and has been validated against whole-body calorimetry and DLW to provide an accurate energy expenditure estimate at a group level. ${ }^{58}$ The Flex HR is commonly defined as the mean of the highest heart rate during rest and the lowest heart rate during light intensity exercise, ${ }^{20,45}$ however, other definitions have been used. Ekelund et al. ${ }^{20}$ compared two Flex HR definitions in a study of young male athletes. The definition just described was termed Flex HR2 and was a higher value than generated by Flex HR1 - the mean of heart rates during all resting activities and lowest heart rate during exercise. In this study eight adolescent male athletes (mean age $18.2 \mathrm{yr}$ ) wore heart rate monitors for eight days of a 10-day DLW measurement period after Flex HRs were determined in the laboratory. As expected the Flex HR1 value was significantly lower than the Flex HR2 value $(P=0.004)$, and this led to a significantly longer time spent in physical activity when calculated by Flex HR1 $(402 \pm 97$ $\mathrm{min} / \mathrm{d})$ compared to Flex HR2 $(297 \pm 97 \mathrm{~min} / \mathrm{d})(\mathrm{P}=0.02)$. Despite these differences, the estimated total energy expenditure did not differ between definitions. Neither Flex HR 
estimate of total energy expenditure differed significantly from that measured by DLW, although the Flex HR2 mean value was closer to the mean DLW value and individual differences were broad for both Flex HR definitions (range $=-10.6 \%$ to $24.2 \%$ ). In this study Ekelund et al. ${ }^{20}$ demonstrated that accurate group estimates of energy expenditure can be generated from the Flex HR method, no matter the definition used. The definition of Flex HR did impact the evaluation of time spent in physical activity, emphasizing the need to use the definition validated in one's study population of interest (e.g., overweight women, children). As with other physical activity energy expenditure assessment methods, the Flex HR was not shown to accurately estimate the energy expenditure of individuals.

Another method developed more recently predicts energy expenditure from heart rate after adjusting for the subject's age and fitness level. Strath et al. ${ }^{67}$ adjusted for age and fitness by expressing the data as a percent of heart rate reserve $(\% \mathrm{HRR})$ and percent of $\mathrm{VO}_{2}$ reserve $\left(\% \mathrm{VO}_{2 \mathrm{R}}\right)$ - all calculations are available in the article. In this study 61 adults participated in a wide range of field and laboratory activities, both inside and outside, while wearing a Polar HR transmitter and the Cosmed $\mathrm{K} 4 \mathrm{~b}^{2}$ indirect calorimeter. The typical relationship between heart rate (beats $/ \mathrm{min})$ and $\mathrm{VO}_{2}(\mathrm{ml} / \mathrm{kg} / \mathrm{min})$ resulted in a modest correlation of $r=0.68$. The relationship between energy expenditure estimated using the age and fitness adjusted values and measured energy expenditure was much tighter $(r=0.87)$. The researchers used established equations to predict the max heart rate and $\max \mathrm{VO}_{2}$ of each subject that were needed in the $\% \mathrm{HRR}$ and $\% \mathrm{VO}_{2 \mathrm{R}}$ calculations. Actually measuring max exercise values would have most likely improved the energy expenditure estimate; however, the estimation method is more practical for large studies and for populations not able to exercise at a maximal intensity. This method shows promise in providing a more accurate use of heart rate to quantify energy expenditure for a group; however, individual values were not provided and most likely have a wide range similar to other methods.

Monitoring heart rate overtime can provide a low cost, noninvasive assessment of physical activity frequency, intensity, and duration. ${ }^{67}$ However the external factors that influence heart rate other than physical activity can complicate the use of this tool. A more significant complication comes when heart rate is used to quantify energy expenditure. Not only are individual calibration curves needed which require laboratory resources, personnel, and time; but age, fitness level, environmental conditions, and the activity performed all 
influence the heart rate-energy expenditure relationship. As more studies are performed and prediction methods are developed, the use of heart rate to estimate energy expenditure at a group level becomes a valid technique. Unfortunately heart rate cannot be used to quantify energy expenditure in large population studies or the energy expenditure of individuals.

\section{Uniaxial Accelerometers}

Accelerometers, compared to pedometers, are more sophisticated motion sensors designed to detect the accelerations of the body. Montoye et al. ${ }^{53}$ stated that the body's accelerations are theoretically proportional to the muscle forces generated in movement and are therefore related to the energy expenditure of the movement. Accelerometers are typically attached close to the center of mass of the body-hip, waist, or low back-because activity energy expenditure is a function of the body's acceleration and displacement of the body's mass. ${ }^{79}$ Unfortunately, most activities are complex, and the energy expenditure of the movement cannot be fully reflected in the acceleration or deceleration of the body's mass. ${ }^{10}$ This limitation will be evident in the following review of research studies. The single-plane or uniaxial accelerometer was developed to quantify physical activity by measuring acceleration in the vertical plane $(\mathrm{z})$. Although differences exist between each device, the general technology is similar. A cantilevered piezoelectric beam senses vertical acceleration in the force range of 0.05 to $2.0 \mathrm{G}$ within a given frequency response range (e.g., 0.25 to 2.5 Hz). ${ }^{5,74}$ This level of sensitivity was chosen in order to avoid recording forms of motion other than human movement (e.g., vibrations from car travel). ${ }^{5,38}$ The monitor contains a microprocessor that filters the acceleration signal and converts the signal to a numeric value, which accumulates over a specified time interval and becomes the raw data value or activity

counts (e.g., counts/min). ${ }^{39}$ Activity counts can be used in a similar manner to step counts or entered into regression equations to estimate energy expenditure. Some devices convert the data to estimates of gross or net energy expenditure themselves (using the entered participant's gender, age, height, and weight to estimate resting metabolic rate), while other monitors require the raw data to be downloaded to a computer for subsequent analysis. ${ }^{5}$ The ability of uniaxial accelerometers to accurately assess physical activity can be determined by examining the output of these monitors compared to indirect calorimetry or DLW measurements. 
Walking is one of the most basic and widely used forms of physical activity, making the validity of the assessment of walking crucial to the usefulness of a motion sensor. Balogun et al. ${ }^{4}$ had 25 adult volunteers walk on a level treadmill during four exercise bouts $(2,3,4$, and $4.8 \mathrm{mph}$ ) for 8 -min each while wearing the Caltrac accelerometer (Hemokinetics, Inc., Madison, WI). An indirect calorimetry system was used simultaneously to measure $\mathrm{VO}_{2}$ (converted to energy expenditure) as the criterion method. A positive correlation was found between the Caltrac output (counts $/ \mathrm{min})$ and $\mathrm{VO}_{2}(\mathrm{ml} / \mathrm{kg} / \mathrm{min})(\mathrm{r}=0.76, \mathrm{p}<0.001)$, and a stronger linear relationship was found between the Caltrac output and energy expenditure $(\mathrm{kcal} / \mathrm{min})(\mathrm{r}=0.91, \mathrm{p}<0.0001)$. However, the difference between the accelerometer estimation of energy expenditure and energy expenditure measured by indirect calorimetry was significant $(\mathrm{p}<0.01)$; the Caltrac overestimated the measured energy expenditure by a range of $13.3 \%$ to $52.9 \%$. Although the raw Caltrac energy expenditure readings-using the manufacturer's proprietary regression equation - significantly overestimated the actual energy expenditure, Balogun et al. ${ }^{4}$ found that the use of appropriate regression equations could result in valid estimates of $\mathrm{VO}_{2}$ or energy expenditure. Similar results were found by Haymes et al. ${ }^{26}$ who also examined the validity of the Caltrac estimation during level walking. In this study, 20 volunteers walked on a treadmill at 2,3,4, and $5 \mathrm{mph}$ while wearing the Caltrac and breathing into a gas analysis system. The correlation between Caltrac activity counts and indirect calorimetry measurements was high $(\mathrm{r}=0.86)$, however, the energy expenditure estimations by the Caltrac were significantly greater than the measured values. The Caltrac significantly $(\mathrm{p}<0.05)$ overestimated the energy expenditure of walking at each speed (average of $1.5 \mathrm{kcal} / \mathrm{min}$ ) except the slowest speed, $2 \mathrm{mph}$. The Caltrac energy expenditure estimation and activity counts increased as speed increased, which was reflected by the high correlation with the indirect calorimetry measurements. Based on the previous study discussed by Balogun et al., ${ }^{4}$ it is likely that more accurate energy expenditure estimates could have been generated if a better regression equation had been used. Two other uniaxial accelerometers, the CSA monitor (Computer Science Applications, Inc., Shalimar, FL) and Biotrainer monitor (IM Systems, Baltimore, MD), were used by Welk et al. ${ }^{74}$ to investigate the assessment of ambulation (walking at $3 \mathrm{mph}$, walking at $4 \mathrm{mph}$, and jogging at $6 \mathrm{mph}$ ) against indirect calorimetry measurements. Fifty-two young to middle-aged adults walked and jogged on a level treadmill at each intensity for 6 minutes. 
Both the CSA and Biotrainer were highly correlated with measured $\mathrm{VO}_{2}$ for the treadmill bouts $(r=0.81$ and $r=0.87$, respectively). The estimated METs from the CSA monitor were not significantly different from the measured METs at any treadmill speed, while the Biotrainer significantly overestimated the measured METs at all three walking speeds (21 to $40 \%, \mathrm{p}<0.01)$. Estimates of METs from the CSA were calculated using the regression equation developed by Freedson et al., ${ }^{22}$ while the Biotrainer estimates came from using the manufacturer's algorithms. Welk et al. ${ }^{74}$ concluded that although the CSA monitor yielded more accurate estimates, the differences could be attributed to a more accurate calibration equation and not the monitors themselves.

Laboratory-based calibration equations appear to be more accurate than the manufacturer-based algorithms for estimating the energy expenditure of walking with uniaxial accelerometers. ${ }^{74}$ Yngve et al. ${ }^{80}$ evaluated the predictive value of four previously published equations designed to convert activity counts into gross energy expenditure estimates (METs). Twenty-eight healthy adults completed a walk/jog protocol on an indoor track while wearing an MTI activity monitor (formerly known as CSA; model WAM 7164; Manufacturing Technology Inc., Fort Walton Beach, FL) attached to the hip. Significant differences were found between the measured METs (using the Cosmed K4 indirect calorimeter) and predicted METs from the equation developed by Freedson et al. ${ }^{22}(\mathrm{P}<0.01)$ and the equation developed by Swartz et al. ${ }^{68}(\mathrm{P}<0.001)$. The METs predicted from the Hendelman et al. ${ }^{27}$ locomotor equation and all activity equation did not differ from the measured METs $(\mathrm{P}=0.41$ and $\mathrm{P}=0.21$, respectively). The researchers attributed these differences in predictive accuracy to the scenarios in which the equations were derived. The Hendelman et al. ${ }^{27}$ locomotor equation was derived from the activity of walking on a track at different speeds - a similar activity and setting to the study being evaluated. The all activity equation of Hendelman et al. ${ }^{27}$ included self-selected intensity walking as well, during the golf and lawn mowing activities assessed. In contrast, the Freedson et al. ${ }^{22}$ equation was developed from treadmill walking and jogging, which has been shown to result in a different accelerometer output compared to a more natural environment (e.g., indoor track). ${ }^{80}$ And the Swartz et al. ${ }^{68}$ equation was developed from a variety of moderate intensity activities. Yngve et al ${ }^{80}$ concluded that the setting in which the predictive equation has been developed impacts the accuracy of the estimates it generates. To improve the chances of making more 
accurate energy expenditure predictions from MTI activity counts, the prediction equation used should be derived from similar activities performed in a similar setting to the activity being assessed.

Welk et al. ${ }^{74}$ did not separate the walking data from the jogging data in the study previously mentioned; in spite of that, it is important to investigate the validity of the uniaxial accelerometer's assessment of jogging and/or running because the mechanics differ from walking. Haymes et al. ${ }^{26}$ had their subjects $(n=20)$ run on a level treadmill at 4, 5, 6, 7, and $8 \mathrm{mph}$ while the Caltrac and indirect calorimetry system assessed the activity. The correlation between Caltrac activity counts and indirect calorimetry measurements was very low $(\mathrm{r}=0.29)$. The Caltrac significantly $(\mathrm{p}<0.05)$ overestimated the energy expenditure of running at 4 to $7 \mathrm{mph}$ but underestimated the running energy cost at $8 \mathrm{mph}$. In general the overestimation improved as speed increased (although there was relatively no difference between 4 and $5 \mathrm{mph}$ ) - $3.6 \mathrm{kcal} / \mathrm{min}$ at $4 \mathrm{mph}, 3.7 \mathrm{kcal} / \mathrm{min}$ at $5 \mathrm{mph}, 2.6 \mathrm{kcal} / \mathrm{min}$ at $6 \mathrm{mph}$, and $1.1 \mathrm{kcal} / \mathrm{min}$ at $7 \mathrm{mph}$. The Caltrac underestimated the energy expenditure of running at $8 \mathrm{mph}$ by $0.74 \mathrm{kcal} / \mathrm{min}$. Haymes et al. ${ }^{26}$ also found that Caltrac did not register significant increases in energy expenditure or activity counts in subjects running between 5 and $8 \mathrm{mph}$, implying the device could not distinguish between these running speeds and intensities and explaining the low correlation. The researchers stated this limitation was most likely due to the little change in stride frequency occurring between these running speeds, resulting in little change in vertical acceleration. In order to detect the changing intensity that does occur in running between 5 and $8 \mathrm{mph}$, an accelerometer measuring acceleration in the horizontal plane would be needed.

Beyond the ambulatory activities, the assessment of other daily living activities is important in determining the usefulness of uniaxial accelerometers. The CSA was used by Hendelman et al. ${ }^{27}$ and was compared against a portable indirect calorimeter (TEEM100, AeroSport, Inc., Ann Arbor, MI) in a study of 25 subjects who played two holes of golf and performed indoor and outdoor household tasks. The measured metabolic cost of the activities was equated to MET levels. The correlation between CSA counts and METs was $r$ $=0.59$ for all activities combined. Hendelman et al. ${ }^{27}$ also assessed the subjects during walking on an indoor track and used individual regression equations developed from the walking data to predict METs from activity counts. The researchers found the CSA 
underestimated the METs of each of the recreational and household activities substantially and significantly ( $31 \%$ to $57 \%$ ). In a study by Welk et al., ${ }^{74} 52$ young to middle-aged adults participated in field activities - mowing, raking, shoveling, vacuuming, sweeping, and stacking groceries - each of 6 minute duration while wearing the CSA and Biotrainer monitors. The indirect calorimetry equipment used during these field activities was the Aerosport KB1-C portable metabolic cart. Both the CSA and Biotrainer were moderately correlated for field activities $(r=0.48$ and $r=0.59$, respectively). Significant underestimation $(p<0.001)$ occurred from both monitors for each of the six field activities; the average degree of underestimation was 53\% for the CSA and 52\% for the Biotrainer. Similar moderate correlations were found by Bassett et al. ${ }^{5}$ when 81 adult volunteers performed between one and nine lifestyle activities ( 28 total). Each activity was performed by 12 subjects for $15 \mathrm{~min}$ and fit into one of the following categories: yard work, occupation, housework, family care, conditioning, and recreation. Each participant wore the Cosmed $\mathrm{K} 4 \mathrm{~b}^{2}$ portable indirect calorimetry system and three uniaxial accelerometers [CSA model 7164, Caltrac, and Kenz (Select 2 model, Nagoya, Japan)]. For all activities combined, the correlation coefficients with indirect calorimetry for each device (using the manufacturer's regression equations) were $\mathrm{r}=0.62$ for CSA, $\mathrm{r}=0.58$ for Caltrac, and $\mathrm{r}=0.55$ for Kenz. The mean error scores (indirect calorimetry minus device) for the three uniaxial accelerometers energy expenditure estimations differed significantly from zero $(\mathrm{P}<0.001)$; they all underestimated the measured METs.

The use of portable indirect calorimetry systems in the above studies have been useful in demonstrating the moderate association between uniaxial accelerometer output and indirect calorimetry measurements $(\mathrm{r}=0.48$ to 0.62$)$ and the high degree of energy expenditure underestimation in activities of daily living. Doubly labeled water studies help to further the evaluation of uniaxial accelerometers in estimating energy expenditure in free-living subjects. Leenders et al. ${ }^{38}$ compared the average daily physical activity energy expenditure derived from the CSA activity monitor to DLW over 7 days in 13 women. The CSA activity monitor recorded activity counts which were converted into an energy expenditure estimate per minute and then per day. The CSA energy expenditure estimates significantly underestimated the DLW physical activity energy expenditure measurements by $59 \%$. Similar underestimations were found by Starling et al. ${ }^{64}$ in a study of older men and women 
( $\mathrm{n}=67)$. Over 9 days the Caltrac accelerometer was found to underestimate daily physical activity energy expenditure by 50-55\% compared to DLW measurements. The Caltrac estimated physical activity energy expenditure was $379 \pm 162 \mathrm{kcal} /$ day for the older women and $554 \pm 242 \mathrm{kcal} /$ day for the older men compared to DLW measurements of $873 \pm 244$ $\mathrm{kcal} / \mathrm{d}$ and $1211 \pm 429 \mathrm{kcal} / \mathrm{day}$, respectively.

The studies that used uniaxial accelerometers to assess the physical activity energy expenditure of daily living activities present a bleak picture of the usefulness of these devices. The main limitation of these monitors in predicting free-living energy expenditure is that no single regression equation can accurately predict energy expenditure based on the activity counts for all activities. ${ }^{5}$ Many activities (e.g., lifting objects, stair climbing) result in very little vertical acceleration but contribute to physical activity energy expenditure. Uniaxial accelerometers, however, do provide an objective assessment of the frequency, intensity, and duration of ambulatory physical activity and can assess the physical activity "pattern" of most populations. ${ }^{5}$ Bassett et al. ${ }^{5}$ used the regression equation of Hendelman et al., ${ }^{27}$ which was based on lifestyle activities, to predict energy expenditure from CSA activity counts generated from subjects performing a variety of lifestyle activities. The use of the equation resulted in an over prediction of energy expenditure in light intensity activity and under prediction of energy expenditure in heavy intensity activity. In spite of this, the equation was useful in identifying intensity "cut-points" and could discriminate between time spent in light, moderate, and vigorous intensity activity. More studies need to be done to evaluate the use of regression equations for different activities and populations.

The ability of uniaxial accelerometers to make valid estimates of energy expenditure during level walking has been inconsistent, but the use of improved regression equations has resulted in valid estimates. ${ }^{4,74}$ Uniaxial accelerometers lack the ability to accurately assess the energy expenditure of running ${ }^{26}$ and are poor measures of energy expenditure in daily living activities, ${ }^{27,74}$ although laboratory-based regression equations would likely improve the energy expenditure estimate of running and lifestyle activities. In free-living situations the underestimation of physical activity energy expenditure was great ${ }^{38,64}$ implying that uniaxial accelerometers are not accurately detecting all movement and are not accurately estimating energy expenditure with the provided equations. These motion sensors cannot accurately assess the energy expenditure involved in common activities, making them limited in their 
assessment of overall daily energy expenditure; however, the pattern of physical activity can be assessed by these devices in most populations. ${ }^{5}$

\section{Triaxial Accelerometers}

Triaxial accelerometers were developed to detect body acceleration in three planes with the intent to improve accuracy as compared to single-plane accelerometers. Triaxial accelerometers contain three piezoceramic elements orthogonally mounted which measure acceleration, when attached to the body, in the anterior-posterior (x), medio-lateral (y), and vertical (z) directions. ${ }^{39}$ Activity data can be collected in 1-min intervals and stored for days before being downloaded on a computer. ${ }^{13,39}$ Computer software packages can provide activity count values for each of the three directions (vectors) and a summary of the three vectors as a vector magnitude $\left(\mathrm{V}_{\text {mag }}\right) .{ }^{39,75}$ Total energy expenditure is calculated from the sum of the basal metabolic rate (estimated from the subject's gender, age, height, and weight) and physical activity energy expenditure (calculated via a proprietary equation using the $\mathrm{V}_{\text {mag }}$ data and subject's body mass). ${ }^{13,39}$ Whether triaxial accelerometers provide a better assessment of physical activity and estimation of energy expenditure than uniaxial accelerometers can be determined by reviewing studies that compare these devices to the gold standard measures of physical activity under a variety of conditions.

A few studies have looked specifically at triaxial accelerometer counts compared to indirect calorimetry energy expenditure measurements. These studies help evaluate the ability of triaxial accelerometers to assess physical activity but do not look at energy expenditure estimates. Levine et al. ${ }^{40}$ compared the Tracmor triaxial accelerometer (Maastricht, The Netherlands) counts to indirect calorimetry. Seven subjects were monitored while walking on a treadmill and while walking on level ground at three different speeds. The Tracmor units (similar to step counts; units/min) were not significantly different for either treadmill or level ground walking at any speed $(\mathrm{r}=1.0)$, implying the Tracmor produced the same output at a given speed regardless of the terrain. For the group, the increase in Tracmor units above resting conditions correlated with the energy expenditure measurement by indirect calorimetry above resting in both walking terrains $\left(\mathrm{r}^{2}=1.0\right)$. Tracmor units increased as velocity increased during level walking. Tracmor units could be reliably converted to velocity using individual regression equations, however, a group regression equation did not provide a valid prediction. Meijer et al. ${ }^{51}$ also found a strong 
linear relationship between triaxial accelerometer (developed in their laboratory) output and measured energy expenditure for each individual subject - the standard error of estimate was $14.4 \mathrm{~J} / \mathrm{min} / \mathrm{kg}$. The 16 subjects performed a range of movement activities, beginning with sitting at rest and ending with treadmill running at $8 \mathrm{mph}$, each for $5 \mathrm{~min}$. When the data from the subjects were pooled, a linear relationship was also found; however, the use of this group regression equation resulted in much greater error (standard error of estimate $=79.1$ $\mathrm{J} / \mathrm{min} / \mathrm{kg}$ ). Triaxial accelerometers can detect primarily ambulatory physical activity, and their outputs are associated with the measured energy expenditure of those activities. However, predictions using the accelerometer output seem to require individual regression equations thus limiting broad applicability.

As the majority of Americans accumulate most of their daily physical activity through walking, the accurate assessment of this activity has practical importance. Sherman et al. ${ }^{63}$ evaluated the walking exercise energy expenditure estimates made by the Tritrac-R3D accelerometer (Reining International, Madison, WI) by comparing them to indirect calorimetry measurements. Sixteen physically active subjects were assessed during treadmill exercise at speeds determined to elicit 40 and $55 \%$ of each individual's peak $\mathrm{VO}_{2}$. The $40 \%$ intensity was a walk for all subjects (average speed of $3.97 \pm 0.44 \mathrm{mph}$ ), while the $50 \%$ intensity may have required some participants to jog slowly (average speed of $5.00 \pm 0.80$ $\mathrm{mph}$ ). The energy expenditure estimated by the Tritrac-R3D at the $40 \%$ and $50 \%$ intensity levels did not differ significantly from the indirect calorimetry measurements $(6.12 \pm 1.24$ $\mathrm{kcal} / \mathrm{min}$ vs. $6.49 \pm 1.50 \mathrm{kcal} / \mathrm{min}$ for $40 \% ; 10.87 \pm 2.91 \mathrm{kcal} / \mathrm{min}$ vs. $9.97 \pm 2.25 \mathrm{kcal} / \mathrm{min}$ for $50 \%$, respectively). The overall accuracy of the TriTrac-R3D measurement was a mean $6 \%$ underestimation for the lower and $9 \%$ overestimation for the higher intensity treadmill walking exercise, although the under- and overestimation was not statistically significant. The Tritrac-R3D energy expenditure estimation was significantly correlated to the energy expenditure measurement at both exercise intensities $(r=0.85$ for $40 \%, r=0.73$ for $55 \%$; $\mathrm{p}<0.05$ ). In another study by Nichols et al., ${ }^{54} 60$ adults completed 5 -min exercise bouts of horizontal walking at 2 and $4 \mathrm{mph}$. The Tritrac-R3D significantly overestimated the energy expenditure of walking on a horizontal surface compared to the measured energy expenditure $(p<0.01)$. The mean and percent differences between indirect calorimetry energy expenditure and Tritrac-R3D energy expenditure were $0.0082 \mathrm{kcal} / \mathrm{kg} / \mathrm{min}$ and $17 \%$ for $2 \mathrm{mph}$ and 0.0241 
$\mathrm{kcal} / \mathrm{kg} / \mathrm{min}$ and $21 \%$ for $4 \mathrm{mph}$. The researchers noted that although the estimated energy expenditure differed statistically from the measured energy expenditure, the difference in practical terms was slight (only 20-40 kcal for a 70-kg person exercising for 30 minutes). Even less positive results were found by Campbell et al. ${ }^{13}$ when 20 women walked on an asphalt track at a self-selected speed while wearing the Tritrac-R3D accelerometer and Cosmed $\mathrm{K} 4 \mathrm{~b}^{2}$ portable indirect calorimetry system. The Tritrac-R3D estimated energy expenditure $(6.19 \pm 0.93 \mathrm{kcal} / \mathrm{min})$ differed significantly from the measured value $(4.74 \pm$ $0.81 \mathrm{kcal} / \mathrm{min})(\mathrm{P}<0.05)$, resulting in an overestimation of approximately $30 \%$. The energy expenditure estimate of walking improved when the raw Tritrac-R3D data was entered into the Chen and Sun ${ }^{15}$ nonlinear energy expenditure model; nonetheless, the adjusted energy expenditure estimate $(5.92 \pm 0.77 \mathrm{kcal} / \mathrm{min})$ still differed significantly from the measured value (overestimation of $25 \%, \mathrm{P}<0.05$ ). Welk et al. ${ }^{74}$ found similar results when they compared the energy expenditure estimate of the Tritrac-R3D monitor (Tritrac) to indirect calorimetry measurements during treadmill exercise in 52 participants, at least 36 completed each trial. The men and women walked on a horizontal treadmill at speeds of $3 \mathrm{mph}$ and 4 $\mathrm{mph}$ - each for $6 \mathrm{~min}$ duration. The Tritrac monitor estimates were highly correlated with measured $\mathrm{VO}_{2}$ for the treadmill exercise (including a jog at $\left.6 \mathrm{mph}\right)(\mathrm{r}=0.93)$, although the energy expenditure of the walking exercise was significantly overestimated (34 to $49 \%$ ) by the Tritrac $(\mathrm{p}<0.05)$. Besides using the manufacturer's algorithms to estimate energy expenditure from Tritrac counts, Welk et al. ${ }^{74}$ also used the Tritrac regression equation developed by Nichols et al. ${ }^{54}$ Use of this regression equation also resulted in a significant overestimation of walking energy expenditure $(p<0.05)$ but to a lesser degree (average of 12 to $18 \%$ ). Bland-Altman plots displayed the tendency of the Tritrac to overestimate the energy expenditure of walking, but they also displayed a much tighter distribution of difference scores (compared to the uniaxial accelerometers studied) implying a stronger predictive accuracy. The energy expenditure estimate of walking on a horizontal surface by triaxial accelerometers was highly correlated to measured energy expenditure in the studies reviewed. The ability of triaxial accelerometers to accurately estimate the energy expenditure of walking varied in the studies, as was the case with uniaxial accelerometers; however, it appears that the use of an appropriate regression equation can generate valid estimates. 
The biomechanics of running differ from that of walking and therefore need to be addressed separately. Sixty adults jogged on a horizontal treadmill for $5 \mathrm{~min}$ (one section of a larger study) in a study by Nichols et al., ${ }^{54}$ while energy expenditure was estimated by the Tritrac-R3D accelerometer (Tritrac) and compared to energy expenditure measurements by indirect calorimetry. The Tritrac significantly overestimated the energy expenditure of jogging by approximately $19 \%$ (mean difference of $0.0320 \mathrm{kcal} / \mathrm{kg} / \mathrm{min}, \mathrm{p}<0.01$ ) compared to the measured energy expenditure. Jakicic et al. ${ }^{34}$ had 18 young adults run on a motorized treadmill for 20 min while wearing two TriTrac-R3D accelerometers and breathing into an indirect calorimetry system. The subjects ran at a speed of $5.0 \mathrm{mph}$ for the entire duration, while the grade changed after $10 \mathrm{~min}$ from $0 \%$ to $5 \%$ to increase the exercise intensity (only 9 subjects completed the higher intensity run). Significant energy expenditure correlations $(\mathrm{p}<0.05)$ were found for running between the Tritrac-R3D and indirect calorimetry-average of $\mathrm{r}=0.89$ for the $0 \%$ grade and $\mathrm{r}=0.80$ for the $5 \%$ grade. Of the two Tritrac-R3D units worn by each subject, one yielded results similar to indirect calorimetry for the lowest intensity of running ( $0 \%$ grade), while the other significantly over-estimated the energy expenditure of this specific activity by $1 \mathrm{kcal} / \mathrm{min}(\mathrm{p}<0.001)$. The energy expenditure of the higher intensity run on the $5 \%$ grade was underestimated significantly by one of the monitors $(-1.6 \pm 0.9 \mathrm{kcal} / \mathrm{min}, \mathrm{p}<0.05)$ and not significantly by the other $(-1.0 \pm 0.8 \mathrm{kcal} / \mathrm{min})$. The total energy expenditure of the 20 min run estimated by the TriTrac-R3D monitors did not differ statistically from that measured by indirect calorimetry (average underestimation was only 4\%). The tendency of the accelerometer to overestimate the energy cost of running on the flat surface and underestimate the energy cost of running on the incline was most likely averaged out to no difference when the two intensities were combined. The Tritrac-R3D was not found to overestimate the energy expenditure associated with running on a flat surface in the study by Sherman et al. ${ }^{63}$ Sixteen active subjects ran on a zero grade treadmill for 15 min at a speed determined to elicit $70 \% \mathrm{VO}_{2 \text { peak }}$ (average speed of $6.28 \pm 1.43 \mathrm{mph}$ ). The TritracR3D energy expenditure estimate $(12.71 \pm 2.38 \mathrm{kcal} / \mathrm{min})$ did not differ from the indirect calorimetry measured energy expenditure $(12.78 \pm 3.02 \mathrm{kcal} / \mathrm{min})$, and the two measures were well correlated $(\mathrm{r}=0.81)$. Welk et al. ${ }^{74}$ had young to middle-aged adults $(\mathrm{n}=36$ to 43 ) jog on a horizontal treadmill at $6 \mathrm{mph}$ for $6 \mathrm{~min}$ while the Tritrac-R3D monitor and an indirect calorimeter assessed energy expenditure. The Tritrac-R3D significantly 
overestimated the measured energy expenditure by 26 to $35 \%(p<0.05)$ using the manufacturer's algorithms, however, the use of the regression equation by Nichols et al. ${ }^{54}$ resulted in no significant difference compared to the measured energy expenditure. The regression equation generated values slightly larger than the measured values (1 to $5 \%)$. Use of a regression equation also generated valid estimates in a study by Campbell et al. ${ }^{13}$ on women $(n=20)$ in a field setting. The Tritrac-R3D energy expenditure estimate was compared to energy expenditure measured by the Cosmed $\mathrm{K} 4 \mathrm{~b}^{2}$ portable indirect calorimeter when subjects jogged at a self-selected speed on an outdoor track. The energy cost of jogging estimated by the Tritrac-R3D using the manufacturer's algorithm $(12.84 \pm 0.91$ $\mathrm{kcal} / \mathrm{min})$ differed significantly from the measured energy expenditure $(11.10 \pm 1.72$ $\mathrm{kcal} / \mathrm{min})(\mathrm{P}<0.05)$. Although the overestimation was significant (approximately $16 \%)$, the intraclass correlation coefficient between the two measures of energy expenditure was fairly strong $(r=0.67)$. Use of the nonlinear model developed by Chen and Sun ${ }^{15}$ resulted in an estimate that did not differ from the measured energy expenditure $(10.84 \pm 1.60 \mathrm{kcal} / \mathrm{min}$ vs. $11.10 \pm 1.72 \mathrm{kcal} / \mathrm{min}$, respectively). Some of the triaxial accelerometer studies have demonstrated the ability of these devices to generate valid energy expenditure estimates for jogging while others only achieved valid estimates using a laboratory-based calibration equation.

Uniaxial accelerometers were not able to detect the increased energy cost of walking on an incline, and researchers speculated that the detection of acceleration in three planes would allow triaxial accelerometers to accurately detect the energy expenditure of inclined walking. Levine et al. ${ }^{40}$ studied the effect of walking on an incline on energy expenditure as measured by indirect calorimetry and Tracmor output. Eight subjects walked at speeds of 1.2, 2.3, and $3.2 \mathrm{mph}$ on a treadmill at zero incline and then repeated the experiment at inclination grades of 17.5 and 22.1 degrees. Levine et al. ${ }^{40}$ found Tracmor output was not sensitive to the increased metabolic cost of walking on an inclination. At each speed the measured energy expenditure increased with each inclination increase, but no change occurred in Tracmor output. Nichols et al. ${ }^{54}$ investigated the ability of the Tritrac-R3D accelerometer to estimate the energy expenditure of walking on an inclination. Sixty adults walked on a treadmill at 4 $\mathrm{mph}$ with a 5\% grade while the Tritrac-R3D estimated and indirect calorimetry measured energy expenditure. Treadmill walking energy expenditure on a $5 \%$ grade was significantly 
underestimated by the Tritrac by roughly $8 \%$ (average mean difference of 0.0107 $\mathrm{kcal} / \mathrm{kg} / \mathrm{min} ; \mathrm{p}<0.01)$. In a study by Jakicic et al., ${ }^{34}$ two Tritrac-R3D accelerometers were worn by 20 young adults while they walked on a motorized treadmill and were assessed by an indirect calorimetry system. The subjects walked at $3 \mathrm{mph}$ for $30 \mathrm{~min}$, and the grade increased every $10 \mathrm{~min}(0 \%$ to $5 \%$ to $10 \%)$. Correlation coefficients between the energy expenditure estimate of the TriTrac-R3D and measurement by indirect calorimetry were significant and similar for each walking intensity and both TriTrac-R3D units (range of $r=$ 0.78 to 0.86 ). The inclined walking energy expenditure was underestimated by both TriTracR3D monitors significantly $(\mathrm{P}<0.001)$ - the average underestimation was $1.3 \mathrm{kcal} / \mathrm{min}$ on the $5 \%$ grade and by $3.6 \mathrm{kcal} / \mathrm{min}$ on the $10 \%$ grade. The energy expenditure of the total walking bout was underestimated significantly by the TriTrac-R3D compared to the measured energy expenditure of indirect calorimetry (average of $21 \%, \mathrm{P}<0.001$ ). Jakicic et al. ${ }^{34}$ analyzed the raw accelerometer data and found that despite the increasing workload during walking (and running as discussed in the previous section) no change occurred in vector magnitude. The TriTrac-R3D was unable to detect the increased workload associated with an inclination.

Much different results were found in a study by Campbell et al., ${ }^{13}$ primarily because the inclined walking protocol differed greatly. Women volunteers $(n=20)$ walked on a 32-m long, 12 degree slope at a pace of their choosing. The walking on an incline involved both ascending and descending which differed from exclusive ascension in other experiments. The energy expenditure of this activity was assessed by the Tritrac-R3D accelerometer and Cosmed $\mathrm{K} 4 \mathrm{~b}^{2}$ portable indirect calorimeter. The mean error score for walking on an incline was not significantly different from zero $(-0.11 \pm 1.44 \mathrm{kcal} / \mathrm{min}, \%$ difference $=-1.7 \%)$. Campbell et al. ${ }^{13}$ speculated that this could have occurred due to the balancing out of energy expenditure underestimation with ascending and overestimation with descending. The correlation coefficient between the two measures was moderate for this activity $(r=0.47)$. Triaxial accelerometers do not appear to accurately detect the increased energy cost of walking up an incline. However, the results found by Campbell et al. ${ }^{13}$ imply that the energy expenditure of free-living outdoor walking with evenly distributed uphill and downhill distances may be accurately estimated by a triaxial accelerometer. 
Beyond strictly locomotor activities, it is important to investigate whether a triaxial accelerometer can accurately assess the energy expenditure of other activities. Jakicic et al. ${ }^{34}$ examined whether the TriTrac-R3D accelerometer could provide a valid estimate of energy expenditure during cycling, stepping, and slideboard exercises at different intensities when compared to indirect calorimetry. Between 13 and 19 young adult volunteers completed each exercise in a $20 \mathrm{~min}$ session (10 $\mathrm{min}$ at each intensity) on separate days. Significant energy expenditure correlations $(p<0.05)$ were found for sliding (ranging from $r=0.68$ to 0.81 ) between the two TriTrac-R3D monitors worn and indirect calorimetry, although the correlations for stepping were lower $(\mathrm{r}=0.54$ to 0.75$)$ and for cycling were not significant (ranging from $r=0.04$ to 0.45 ). The TriTrac-R3D accelerometers significantly underestimated energy expenditure compared with indirect calorimetry for all of the exercises-difference scores were -2.8 to $-3.6 \mathrm{kcal} / \mathrm{min}$ for sliding, -2.1 to $-3.1 \mathrm{kcal} / \mathrm{min}$ for stepping, and -3.9 to $-5.5 \mathrm{kcal} / \mathrm{min}$ for cycling ( $\mathrm{p}<0.05$ for all). Total energy expenditure during each exercise was significantly underestimated $(p<0.001)$ for all activities; average underestimations were $35 \%$ for stepping, $41 \%$ for sliding, and $68 \%$ for cycling. For all exercises the energy expenditure estimation was underestimated to a greater degree as intensity increased. However, analysis of the raw vector data revealed that each vector showed a significant increase as intensity increased during each of the three exercises $(\mathrm{P}<0.006)$. A similar experiment was performed by Campbell et al. ${ }^{13}$ on women in a field setting. The Tritrac-R3D (Tritrac) energy expenditure estimate was compared to energy expenditure measured by the Cosmed $\mathrm{K} 4 \mathrm{~b}^{2}$ in 20 women performing a routine of activities (stair climbing, stationary cycling, and arm ergometry) each for 5 minutes; the intensity of each activity was self-selected. The mean error scores (indirect calorimetry minus Tritrac) for all activities were significantly different from zero $(p<0.05)$. The mean differences $( \pm$ SD) and percent differences were large for stair climbing $(2.76 \pm 0.94 \mathrm{kcal} / \mathrm{min}, 33 \%)$, cycling $(2.75 \pm 0.66 \mathrm{kcal} / \mathrm{min}, 53 \%)$, and arm ergometry $(1.20 \pm 0.67 \mathrm{kcal} / \mathrm{min}, 45 \%)$, displaying the great underestimation of energy expenditure by the Tritrac in these activities. The researchers anticipated that the energy expenditure of stationary cycling and arm ergometry would be underestimated by the accelerometer because they are nonweight-baring activities. Intraclass correlation coefficients between the two measures were moderate to weak for all activities $(r=0.50$ for stair climbing, $r=0.41$ for cycling, and $r=0.29$ for arm 
ergometry). Bland-Altman plots were also created to assess agreement between the two measures. There was much discrepancy between the two measures for each of the activities performed, however, no numerical values were provided (e.g., limits of agreement, bias). Welk et al. ${ }^{74}$ evaluated the validity of the Tritrac-R3D monitor (Tritrac) in estimating energy expenditure during lifestyle activities (mowing, raking, shoveling, vacuuming, sweeping, and stacking groceries) compared to energy expenditure measurements of indirect calorimetry. Fifty-two young to middle-aged adults ( 21 men, 31 women) performed each activity for 6 min while wearing the Tritrac and Aerosport KB1-C portable indirect calorimeter. The Tritrac output was only moderately correlated $(\mathrm{r}=0.59)$ with the measured $\mathrm{VO}_{2}$, and the energy expenditure of the lifestyle activities was significantly underestimated by the Tritrac (average of $37 \%$ using the manufacturer's algorithm and $45 \%$ using the regression equation by Nichols et al. $\left.{ }^{54}\right)$. The underestimation by the Tritrac was significant $(\mathrm{p}<0.05)$ for all activities using both prediction equations. The Tritrac energy expenditure underestimation was greater than $36 \%$ for all of the lifestyle activities (using both regression methods), with the exception of lawn mowing. The lawn mowing energy expenditure was underestimated by approximately $9 \%$ using the manufacturer's equation and $26 \%$ using the regression equation. ${ }^{54}$ Lawn mowing most likely resulted in the least underestimation because it involved the most locomotor activity. The detection of movement in three planes does not appear to improve the accuracy of the triaxial accelerometer in estimating the energy expenditure of non-locomotor activities.

Campbell et al. ${ }^{13}$ found the Tritrac provided a poor estimate of energy expenditure during individual activities (walking, jogging, stair climbing, walking on an incline, stationary cycling, and arm ergometry) performed in a field setting. However, when the total routine (activities plus walking between activities) was analyzed, the mean error score was not significantly different from zero $(7.6 \pm 26.99 \mathrm{kcal})$ and the percent difference was low $(3 \%)$. These finding support the possibility that the Tritrac may be useful in estimating energy expenditure over a whole day when estimation errors average out. Two studies have explored this possibility by comparing triaxial accelerometer energy expenditure estimates over full days compared to measurements made by a room calorimeter or DLW. Chen and Sun ${ }^{15}$ had 125 subjects wear a Tritrac-R3D monitor and live individually in a whole-room indirect calorimeter on two different days (24-hours each) — one normal activity day and one 
day involving an exercise protocol. The equation used by the Tritrac to estimate an individual's resting energy expenditure accurately predicted the measured resting energy expenditure $(p=0.822)$. The accuracy of the resting energy expenditure estimation led to significant correlations between estimated and measured total energy expenditure for the exercise day $(\mathrm{r}=0.93)$ and normal day $(\mathrm{r}=0.86)$. However, the Tritrac significantly underestimated the total energy expenditure for both days (approximately $17 \%$ and $14 \%$, respectively; $\mathrm{p}<0.001)$. The Tritrac was even less accurate in estimating physical activity energy expenditure for the exercise day $(\mathrm{r}=0.74 ; 51 \%$ underestimation $)$ and normal day $(\mathrm{r}=$ $0.54 ; 67 \%$ underestimation). In the assessment of physical activity in free-living conditions, Leenders et al. ${ }^{38}$ had 13 healthy women wear a Tritrac-R3D accelerometer for 7 days while undergoing a DLW measurement. The Tritrac-R3D accelerometer significantly underestimated $(35 \%, \mathrm{p}<0.05)$ physical activity energy expenditure measured by DLW. Mean physical activity energy expenditure values $( \pm$ SE) were $478 \pm 51 \mathrm{kcal} / \mathrm{d}$ for the Tritrac-R3D and $798 \pm 83 \mathrm{kcal} / \mathrm{d}$ for DLW. Triaxial accelerometers may be useful in distinguishing between periods of activity and inactivity but have not been proven to accurately estimate daily energy expenditure.

One study also looked at whether the triaxial accelerometer could accurately estimate energy expenditure immediately following exercise. Sherman et al. ${ }^{63}$ found that energy expenditure during post-exercise recovery (following 15 minutes of treadmill exercise at 40, 55 , and $70 \%$ peak $\mathrm{VO}_{2}$ ) was significantly underestimated by the Tritrac-R3D. Only $34 \%$ of the post-exercise energy expenditure was accounted for by the Tritrac-R3D estimations. The inability to detect the elevated metabolic state following high intensity exercise would not be problematic for the majority of the U.S. population who are sedentary and do not partake in high intensity activity, whereas the energy expenditure of individuals in training (those who perform high intensity exercise) would be underestimated.

As it is important to investigate the validity of a measurement tool's estimate, the reliability of its estimate is also important. Nichols et al. ${ }^{54}$ had 20 of the original 60 subjects repeat the identical activity protocol (walking and jogging on a treadmill at varying speeds and grade) on a different day; and on this repeat visit, subjects wore two Tritrac-R3D monitors - one on each hip. In comparing day 1 and day 2 (values from right hip), the intraclass correlation coefficient for the Tritrac-R3D vector magnitude was highest at $2 \mathrm{mph}$ 
$(r=0.92)$ and remained high for the elevated intensities (range of $r=0.87$ to 0.89$)$. The interinstrument reliability was also high when comparing the right and left hip vector magnitude values (range of $r=0.73$ at $6 \mathrm{mph}$ to $\mathrm{r}=0.87$ at $2 \mathrm{mph}$ ). Jakicic et al. ${ }^{34}$ also found significant correlation coefficients in energy expenditure estimates from the two TriTracR3D accelerometers worn by each of their subjects $(n=20)$; correlations were significant for all activities and workloads $(\mathrm{P}<0.05)$. Throughout each 20 to $30 \mathrm{~min}$ activity, the ranges of correlation coefficients between TriTrac-R3D monitors were as follows: $0.76-0.87$ for walking, 0.80-0.92 for running, 0.42-0.88 for stepping, 0.75-0.87 for stair climbing, and 0.54-0.89 for cycling. Triaxial accelerometers appear to provide reliable outputs and energy expenditure estimates for a variety of activities performed at changing intensities.

Triaxial accelerometers have been shown to be better estimators of energy expenditure than the previous tools discussed, but they still have their limitations. Triaxial accelerometers not only underestimate high intensity running energy expenditure ${ }^{34,51}$, but they also cannot detect the increased energy expenditure of walking on an incline ${ }^{40,54}$ or recovering from exercise. ${ }^{63}$ Non-ambulatory physical activity such as cycling ${ }^{13,34}$ and some activities in daily life ${ }^{13,39,74}$ also cannot be adequately detected by triaxial accelerometers, which leads to an inaccurate energy expenditure estimation. Welk et al. ${ }^{74}$ stated that more accurate energy expenditure estimations by some monitors compared to others "may be due to differences in the accuracy of prediction equations rather than differences in technology." Chen and $\operatorname{Sun}^{15}$ found that the individual and general models they developed showed significant improvements in estimation over the manufacturer's model. More laboratorybased calibration studies could enhance the accuracy of the accelerometer as an energy expenditure estimation tool. According to Jakicic et al. ${ }^{34}$ and Welk et al., ${ }^{74}$ accelerometers may be useful in identifying acute periods of physical activity or differentiating between individuals on the basis of typical activity level; but accelerometers have been proven inaccurate in the ability to quantify absolute energy expenditure. 
Triaxial Accelerometers vs. Indirect Calorimetry (IC) Experiments

\begin{tabular}{|c|c|c|c|c|c|c|}
\hline $\begin{array}{l}\text { Author/ } \\
\text { device }\end{array}$ & $\mathbf{n}$ & Context & $\begin{array}{l}\text { Time } \\
(\min )\end{array}$ & Results Estimation & $\mathbf{r}$ & $\begin{array}{l}\text { IC } \\
\text { duration }\end{array}$ \\
\hline $\begin{array}{l}\text { Meijer et } \\
\text { al. }^{51} \\
\text { Lab made }\end{array}$ & 16 & $\begin{array}{l}\text { Sit relaxed } \\
\text { Stand/sit } \\
\text { Standing } \\
\text { Slow steps } \\
\text { Walk, 2mph } \\
\text { Walk/Run faster } \\
\text { Run } 8 \mathrm{mph}\end{array}$ & $\begin{array}{l}5 \\
5 \\
5 \\
5 \\
5 \\
5 \\
5\end{array}$ & $\begin{array}{l}\text { Strong linear rlshp between acc. } \\
\text { counts and EE (individual and } \\
\text { pooled data) } \\
\text { U-est during run }\end{array}$ & & $\begin{array}{l}\text { Continuous } \\
\text { (used last } \\
2-3 \text { min of } \\
\text { each } \\
\text { segment) }\end{array}$ \\
\hline $\begin{array}{l}\text { Sherman et } \\
\text { al. }{ }^{63} \\
\text { Tritrac- } \\
\text { R3D }\end{array}$ & 16 & $\begin{array}{l}\text { Sit at rest } \\
\text { Walk } 40 \% \\
\text { Walk/Run } 55 \% \\
\text { Run } 70 \% \\
\text { Rest (post-ex) }\end{array}$ & $\begin{array}{l}10 \\
15 \\
15 \\
15 \\
20\end{array}$ & $\begin{array}{l}\text { No sig U- or O-est for rest or } \\
\text { exercise } \\
\text { ( } 4 \text { to } 15 \% \text { ) } \\
\text { U-est by } 34 \%\end{array}$ & $\begin{array}{l}0.79 \\
0.85 \\
0.73 \\
0.81 \\
0.60\end{array}$ & $\begin{array}{l}\text { final } 5 \mathrm{~min} \\
\text { final } 10 \mathrm{~min} \\
\text { final } 10 \mathrm{~min} \\
\text { final } 10 \mathrm{~min} \\
\text { all } 20 \mathrm{~min}\end{array}$ \\
\hline $\begin{array}{l}\text { Jakicic et } \\
\text { al. }^{34} \\
\text { Tritrac- } \\
\text { R3D }\end{array}$ & $\begin{array}{l}13- \\
20\end{array}$ & $\begin{array}{l}\text { Walk total } \\
\text { Walk, 3mph } 0 \% \\
\text { Walk, 3mph } 5 \% \\
\text { Walk, 3mph } 10 \% \\
\text { Run Total } \\
\text { Run, } 5 \mathrm{mph} 0 \% \\
\text { Run, } 5 \mathrm{mph} 5 \% \\
\text { Cycle Total } \\
\text { Cycle, 50rpm } \\
\text { Cycle, 65rpm } \\
\text { Step Total } \\
\text { Step 20cycl/min } \\
\text { Step 30cycl/min } \\
\text { Slide Total } \\
\text { Slide } 17 \mathrm{cycl} / \mathrm{min} \\
\text { Slide } 21 \mathrm{cycl} / \mathrm{min}\end{array}$ & $\begin{array}{l}30 \\
10 \\
10 \\
10 \\
20 \\
10 \\
10 \\
20 \\
10 \\
10 \\
20 \\
10 \\
10 \\
20 \\
10 \\
10\end{array}$ & $\begin{array}{l}\text { U-est } 16-26 \% \\
\text { O-est } \\
\text { U-est } \\
\text { U-est } \\
\text { No sig. diff }(2-6 \%) \\
\\
\text { U-est } 67-69 \% \\
\text { U-est } \\
\text { U-est } \\
\text { U-est } 32-37 \% \\
\text { U-est } \\
\text { U-est } \\
\text { U-est } 38-44 \% \\
\text { U-est } \\
\text { U-est }\end{array}$ & $\begin{array}{l}0.83 \\
0.81 \\
0.78 \\
\\
0.89 \\
0.80 \\
\\
0.41 \\
0.11 \\
\\
0.69 \\
0.65 \\
\\
0.81 \\
0.69\end{array}$ & $\begin{array}{l}\text { Continuous } \\
\text { (used last } 5 \\
\text { min of each } \\
10 \text { min } \\
\text { interval) }\end{array}$ \\
\hline $\begin{array}{l}\text { Nichols et } \\
\text { al. } \\
\text { Tritrac- } \\
\text { R3D }\end{array}$ & 60 & $\begin{array}{l}\text { Walk, } 2 \mathrm{mph} \\
\text { Walk, 4mph } \\
\text { Walk, 6mph } \\
\text { Walk, 4mph } 5 \%\end{array}$ & $\begin{array}{l}5 \\
5 \\
5 \\
5 \\
\text { random } \\
\text { order }\end{array}$ & $\begin{array}{l}\text { Est. in } \mathrm{kcal} / \mathrm{kg} / \mathrm{min} \\
\text { O-est } 17 \% \\
\text { O-est } 21 \% \\
\text { O-est } 19 \% \\
\text { U-est } 8 \%\end{array}$ & & $\begin{array}{l}\text { Used } \\
\text { minutes } 4 \\
\text { and } 5\end{array}$ \\
\hline $\begin{array}{l}\text { Welk et } \\
\text { al. }^{74} \\
\text { Tritrac- } \\
\text { R3D }\end{array}$ & 52 & $\begin{array}{l}\text { Walk, 3mph } \\
\text { Walk, 4mph } \\
\text { Jog, 6mph } \\
\text { Field activity (6) }\end{array}$ & $\begin{array}{l}6 \\
6 \\
6 \\
6 \text { each }\end{array}$ & $\begin{array}{l}\text { O-est } 38 \%[14 \%] \\
\text { O-est } 47 \% \text { [18\%] } \\
\text { O-est } 31 \% \text { [no sig diff, 3\%] } \\
\text { U-est } 37 \% \text { [45\%] } \\
\text { [regression equation by Nichols } \\
\text { et al. (1999)] }\end{array}$ & $\begin{array}{l}0.93 \\
\text { for all } \\
\operatorname{Tr} \\
0.59\end{array}$ & Continuous \\
\hline $\begin{array}{l}\text { Levine et } \\
\text { al. }^{40} \\
\text { Tracmor }\end{array}$ & 7 & $\begin{array}{l}\text { Standing } \\
\text { Walk } 1.2 \mathrm{mph} \\
\text { Walk } 2.3 \mathrm{mph} \\
\text { Walk } 3.2 \mathrm{mph}\end{array}$ & $\begin{array}{l}20 \\
14 \\
14 \\
14\end{array}$ & $\begin{array}{l}\text { Increasing acc. counts matched } \\
\text { increasing EE }\end{array}$ & $\begin{array}{l}1.0 \text { for } \\
\text { group }\end{array}$ & Continuous \\
\hline
\end{tabular}




\begin{tabular}{|c|c|c|c|c|c|c|}
\hline & & (Tr and ground) & & & & \\
\hline $\begin{array}{l}\text { Levine et } \\
\text { al. }^{40} \\
\text { Tracmor }\end{array}$ & 8 & $\begin{array}{l}\text { Standing } \\
\text { Walk } 1.2 \mathrm{mph} \\
\text { Walk } 2.3 \mathrm{mph} \\
\text { Walk } 3.2 \mathrm{mph} \\
\text { (repeated at } \\
17.5 \% \text { and } \\
22.1 \% \text { ) }\end{array}$ & $\begin{array}{l}\text { Not } \\
\text { given }\end{array}$ & $\begin{array}{l}\text { No change in acc. counts with } \\
\text { incline }\end{array}$ & & Continuous \\
\hline $\begin{array}{l}\text { Campbell } \\
\text { et al. } \\
\text { Tritrac- } \\
\text { R3D }\end{array}$ & 20 & $\begin{array}{l}\text { Walk } \\
\text { Jog } \\
\text { Stairs (up \& dn) } \\
\text { Walk 12\% (up \& } \\
\text { dn) } \\
\text { Cycle, 50W } \\
\text { Arm ergometry } \\
\text { Total routine } \\
\text { (all speeds self- } \\
\text { selected) }\end{array}$ & $\begin{array}{l}5 \\
5 \\
5 \\
5 \\
\\
5 \\
5 \\
39-42\end{array}$ & 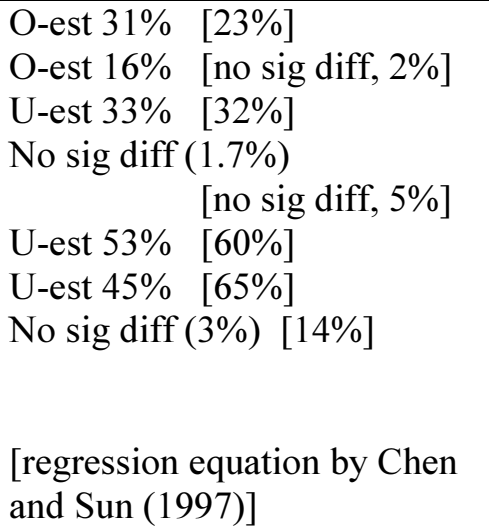 & $\begin{array}{l}0.57 \\
0.67 \\
0.50 \\
0.47 \\
0.41 \\
0.29 \\
0.48\end{array}$ & $\begin{array}{l}\text { Continuous } \\
\text { (used } \\
\text { minutes 2- } \\
4 \text { ) }\end{array}$ \\
\hline
\end{tabular}

Key: U-est $=$ significant underestimation, O-est $=$ significant overestimation, sig $=$ significant, diff $=$ difference 


\section{Use of Multiple Tools}

Conceptually the use of multiple tools (e.g., HR monitor and motion sensor, multiple motion sensors) could be advantageous in the estimation of energy expenditure compared to the use of a single tool. Some research has shown this to be true, although the measurement and analysis processes are more involved.

The use of a HR monitor, motion sensor (or two), and individualized regression equation has been shown to generate a valid estimate of energy expenditure for a variety of activities. In a study by Rennie et al., ${ }^{58}$ a novel instrument that consisted of a HR monitor and simple movement sensor in one chest belt $(\mathrm{HR}+\mathrm{M})$ was used on eight subjects. A calibration test was performed on each subject to determine his or her $\mathrm{VO}_{2}-\mathrm{HR}$ relationship. Inspired and expired $\mathrm{O}_{2}$ concentrations were measured with an $\mathrm{O}_{2}$ analyzer, and $\mathrm{HR}$ was measured while the subject rested in prone and seated positions and exercised on a cycle ergometer in an incremental protocol. The resting values were averaged to provide mean resting energy expenditure. Segmented calibration generated two slopes-one for low and one for moderate-high intensity activity. Each subject then spent 24 hours in a whole-body indirect calorimeter while wearing the HR $+\mathrm{M}$ following multiple protocols involving rest, sedentary activities, and exercise. The calorimeter provided 30-min averages of energy expenditure, so the energy expenditure estimates from the $\mathrm{HR}+\mathrm{M}$ and calibration equations were averaged into 30-min periods. Rennie et al. ${ }^{58}$ found the use of $\mathrm{HR}+\mathrm{M}$ with calibration equations accurately represented the pattern of activity and accurately estimated energy expenditure throughout the day. The estimation of energy expenditure with the HR $+M$ method compared to energy expenditure measured by the calorimeter resulted in a mean percentage error of $0.00 \%(\mathrm{SD}=12.5 \%)$. Individual estimations were better for some subjects than others - percent error ranged from 0.0 to $21.7 \%$.

A variation of this method also produced accurate energy expenditure estimates. Strath et al. ${ }^{66}$ used a HR monitor, two CSA accelerometers, and individualized regression equations to estimate energy expenditure compared to energy expenditure measured by the Cosmed $\mathrm{K} 4 \mathrm{~b}^{2}$. Ten participants each completed submaximal treadmill and arm ergometer tests while HR and $\mathrm{VO}_{2}$ were measured simultaneously. Individualized regression equations were developed for the $\mathrm{HR}-\mathrm{VO}_{2}$ relationship for leg activity and arm activity. Each participant was monitored over a 6-hour period during his or her normal daily routine (at home or work) 
with the HR monitor, two CSA accelerometers (placed at the wrist and thigh), and portable indirect calorimetry unit. Minute-by-minute data was collected by each device. The CSA monitor counts were used to distinguish between periods of rest and activity and whether the activity consisted of arm activity, leg activity, or both. The corresponding HR could be used in the proper regression equation to generate an estimate of energy expenditure. Strath et al ${ }^{66}$ found that the mean minute-by-minute error score for this technique (HR + motion sensor) was not significantly different from zero ( $0.0 \mathrm{METs} ; 95 \% \mathrm{CI}= \pm 1.3 \mathrm{METs})$. The total energy expenditure values estimated did not differ significantly from those measured by indirect calorimetry (748 $\pm 178 \mathrm{METs} / \mathrm{min}$ vs. $749 \pm 138 \mathrm{METs} / \mathrm{min}$, respectively). This technique also provided an accurate estimation of time spent in resting/light, moderate, and hard activity compared to the indirect calorimetry measurements (mean error scores \pm SD: 12 $\pm 19 \mathrm{~min},-9 \pm 16 \mathrm{~min},-3 \pm 5 \mathrm{~min}$, respectively; all $\mathrm{p}>0.05$ ).

Research performed in the same laboratory using the same HR + motion sensor technique also found valid results. This study was unique because it compared the HR + motion sensor technique to the estimates provided by the individual tools. Strath et al. ${ }^{65}$ performed submaximal treadmill and arm ergometer tests on 30 participants to generate individualized regression equations. The participants then performed a range of activities, each for 15 min —housework $(n=11)$, yardwork $(n=9)$, or conditioning $(n=10)$-while wearing three CSA accelerometers (placed on the hip, wrist, and thigh), a HR monitor, and the Cosmed $\mathrm{K} 4 \mathrm{~b}^{2}$. The relationship between the hip-mounted CSA MET values and measured METs was $\mathrm{R}^{2}=0.54$. The measured MET values were significantly underestimated by the hip CSA (mean of 1.1 METs or 29.5\%; $\mathrm{p}<0.001$ ). The use of $\mathrm{HR}$ with the treadmill test $\mathrm{HR}-\mathrm{VO}_{2}$ relationship resulted in a better relationship between predicted and measured METs $\left(\mathrm{R}^{2}=\right.$ 0.67), however, this method overestimated the measured METs, as well, by an average of 0.4 METs or $11.1 \%(\mathrm{p}<0.001)$. The best results were generated from the HR + motion sensor technique with an $\mathrm{R}^{2}=0.81$ and no significant under- or overprediction of measured METs.

The use of individualized prediction equations with multiple assessment devices has been shown to generate accurate estimates of energy expenditure. The practicality of this method would be enhanced if a group prediction equation could generate similar results. This was investigated in a study by Haskell et al. ${ }^{24}$ In this study 17 males performed six submaximal exercise tests while connected to a Vitalog monitor containing a 6-lead ECG and two motion 
sensors (wrist and thigh placement) and an indirect calorimetry system. The six submaximal tests included 1) walking with increasing speed, 2) walking with increasing grade, 3) arm cranking with increasing rpm, 4) cycling with increasing resistance, 5) Air-Dyne cycling with increasing rpm, and 6) bench stepping with increasing load carried. Regression analyses were performed for each individual and for all individuals grouped together. In using the $\mathrm{HR}$, leg motion, and arm motion data for all activities to predict $\mathrm{VO}_{2}$ with each individual's regression analyses, Haskell et al. ${ }^{24}$ found a mean $\mathrm{R}^{2}=0.89$ (range from 0.86 to 0.93 ) and a mean $\mathrm{SEE}=2.3 \mathrm{ml} / \mathrm{kg} / \mathrm{min}$. When single regression analysis was performed on the pooled data, the $\mathrm{R}^{2}$ decreased to 0.73 and the SEE increased to $5.2 \mathrm{ml} / \mathrm{kg} / \mathrm{min}$, demonstrating that better predictions resulted when individual regression equations were used. A similar study done by Luke et al. ${ }^{46}$ resulted in even lower values when all subjects $(n=10)$ data from the activities of daily living circuit (ADLC) and treadmill tests were pooled. The relationship of $\mathrm{VO}_{2}$ from $\mathrm{HR}$ and motion was $\mathrm{R}^{2}=0.45$ when the group equation was used compared to $\mathrm{R}^{2}=$ 0.86 for the ADLC and $\mathrm{R}^{2}=0.90$ for the treadmill tests when individual calibration equations were used.

The results of the aforementioned studies showcase that the simultaneous use of multiple motion sensors, a HR monitor, and an individualized regression equation provides a valid method of assessing physical activity patterns and energy expenditure. Unfortunately this method is time and resource intensive, making application limited. ${ }^{66}$ To use this method, each individual needs to perform submaximal tests in a laboratory using indirect calorimetry equipment and wearing a HR monitor and motion sensor (often two), which leads to increased time and cost. The data analysis is also quite cumbersome and lengthy. The use of group regression equations would simplify this technique, but group regression equations have not been found to produce valid energy expenditure estimations. ${ }^{24,46,58}$

\section{SenseWear ${ }^{\mathrm{TM}}$ Armband Description}

The SenseWear ${ }^{\mathrm{TM}}$ Armband was designed to overcome the limitations of the aforementioned energy expenditure assessment tools. BodyMedia, Inc. (Pittsburgh, PA) has compiled multiple sensor arrays into one compact, wearable device (the SenseWear Armband) designed to gather and analyze physiologic data. The multiple sensors include a two-axis accelerometer, heat flux sensor, skin temperature thermistor, near-body ambient temperature sensor, galvanic skin response sensor (GSR), and optional heart rate (HR) 
receiver. The sensors provide the device with six different streams of raw physiological data, which are analyzed and interpreted into 30 channels of data. ${ }^{42}$ The data obtained from the microprocessor in the SenseWear Armband can be uploaded on BodyMedia's InnerView ${ }^{\mathrm{TM}}$ Research Software computer program for analysis and graph reports of measured and derived data. According to literature from the company, the SenseWear Armband's proprietary algorithms combine the multiple sensor arrays with individual body measurements (gender, age, height, and weight) to calculate the energy expenditure of a full range of free-living activities. $^{43}$ As no research literature has been published on the SenseWear Armband to date, all of the information on the SenseWear Armband has come from internal documents available on the manufacturer's website.

After completing internal studies and developing several prototypes, BodyMedia, Inc. chose the upper arm (the triceps area) to be the location for wearing the SenseWear Armband. ${ }^{35}$ The upper arm was an area that could sense all the physiological information from the sensors. The upper arm was also chosen because it has a similar size and shape for men and women, provides a relatively large surface area for the sensors, is an area of low movement, and provides a site for easy attachment and detachment. The upper arm was also a suitable location because it provides adequate structure and strength to allow the device to be worn with minimal fatigue, and this area is rarely used for other accessories and is often concealed by clothing.

The SenseWear Armband is 3.4 inches long, 2.1 inches wide, and 0.8 inches deep and weighs $3.0 \mathrm{oz}(85 \mathrm{~g})$. Data can be collected continuously for up to four days, using the default settings, without recharging the battery. ${ }^{7}$ The SenseWear Armband contains flexible wings on either side of the monitor to stabilize the monitor, create equal pressure on the sensors, and accommodate virtually all arm sizes and movements. The armband is held on the back of the upper arm with an adjustable elastic strap. BodyMedia, Inc. has tested the wearability of the SenseWear Armband on subjects performing a range of activities (from sleep to extreme activity) and found very little reported discomfort or interference in activity. $^{35}$

The SenseWear Armband monitors physical activity and energy expenditure through its use of multiple sensors. The degree in which each sensor contributes to an energy expenditure calculation is proprietary, although the function of each sensor ${ }^{43}$ is provided 
below. The two-axis accelerometer (measuring vertical and medio-lateral accelerations) in the SenseWear Armband is designed to detect motion and to relate that motion proportionally to generated muscle force, resulting in an energy expenditure estimation. This particular accelerometer also measures the force of gravity on the body, which leads to a prediction of the context in which the subject is operating — standing, reclining, sitting, walking, running, and biking. Context detection allows more models to be developed and allows for complex situations to be modeled more effectively, assuming the contexts are detected accurately. If the context were not detected properly, an incorrect estimation model would be used to generate the energy expenditure estimate. This feature is intended to improve the energy expenditure estimation; however, this feature alone could greatly influence, positively or negatively, the generated estimates.

No research or information could be found in published literature on other two-axis accelerometers to compare their accuracy with triaxial accelerometers. The two-axis accelerometer in the SenseWear Armband is not believed to be less accurate because it is worn on the upper arm, which allows for greater movement than accelerometers worn on the trunk. Since more movement occurs at the arm, the measurement of movement in only two planes could prevent the overestimation of total body acceleration.

Heat production is directly related to metabolic rate. The heat flux sensor in the SenseWear Armband is designed to measure the amount of heat released from the body. This proprietary sensor specifically measures the representative values of heat convection (through the skin being in contact with the air) that are part of the total thermal energy emitted by the body. ${ }^{43}$ The sensor is located in a "thermally conductive path" between the skin and the exposed side of the armband. Close et al. ${ }^{16}$ compared heat loss measurements made by heat-flow meters to those made by a whole-body calorimeter and found that heatflow meters could generate a useful estimate of total heat loss, especially during low activity when the evaporative component of heat loss would not interfere in the measurement. Close et al. ${ }^{16}$ concluded that heat-flow meters could be useful in estimating energy expenditure in low activity periods when other sensors, such as HR monitors, are less useful. However, the role that the SenseWear Armband heat flux sensor plays in the manufacturer's energy expenditure estimation models is proprietary. 
The near-body ambient temperature sensor detects the air temperature immediately around the SenseWear Armband and is attached to the heat flux sensor. The function of this sensor is to detect the rate of change in near-body ambient temperature (temperature at the outer edge of the heat flux sensor) in order to assess the current physical activity being done, variations in environmental conditions, and to verify whether the heat flux sensor is receiving disturbed signals. The ability of this sensor to monitor environmental conditions is important because research has shown the energy expenditure of human subjects increases upon exposure to colder temperatures. ${ }^{18,73}$

Two other sensors, the skin temperature thermistor and galvanic skin response sensor (GSR), take measurements from the protected underside of the unit while in direct contact with the user's skin. Although temperature within the body can vary greatly, especially under extreme conditions, under normal conditions the difference between the core and skin temperature approximates 4 degrees $\mathrm{C}^{57}$ The continuous measurement of skin temperature is important as it tends to reflect core body temperature under normal conditions. According to Gonzalez-Alonso et al., ${ }^{23}$ heat production increases throughout exercise, affecting the core body temperature and heat released to the skin (detected with the skin temperature sensor) and environment (detected with the heat flux sensor). As body temperature increases from increased physical activity or emotional stimuli, one's metabolic rate (or energy expenditure) increases and sweating often occurs. The GSR uses two stainless steel electrodes to measure the electrical conductance of the skin between the two contacts, which is affected by sweat gland activity and pore size. The GSR in the SenseWear Armband can be an indicator of the onset, peak, and recovery of maximal sweat rates. However, this sensor alone cannot distinguish between sweat from physical activity and sweat from emotions such as pain, anger, or surprise.

The SenseWear Armband contains a receiver that can detect heartbeats from a chest strap (similar to the watch that accompanies the chest strap). The HR receiving function, although optional, looks to be advantageous. Alone HR monitors and motion sensors (accelerometers) are limited in their energy expenditure prediction capabilities, but as research in the previous section demonstrated, when used together a better prediction of energy expenditure can result. Heart rate is not included in the manufacturer's energy expenditure estimation models to date, since it is an optional function. However, HR may be beneficial in context detection 
by providing insight into the frequency, intensity, and duration of activity and in energy expenditure estimation as previously noted.

Together the multiple sensors on the SenseWear Armband should theoretically provide a more accurate assessment of energy expenditure, compared to other devices currently available. BodyMedia, Inc. has performed some research on the intra-person repeatability of the SenseWear Armband and provided a summary of the results. ${ }^{44}$ When 40 subjects of varying ages, sexes, heights, and weights wore one armband on each arm during a range of activities (a 150-min protocol involving segments of resting, sitting, standing, walking, and cycling) completed on two separate occasions, the correlation coefficient for the accelerometer was 0.89 and for the GSR sensor was 0.80 . The relationship implies high repeatability but the correlation was not perfect. BodyMedia, Inc. stated this could be because the movements of individual's right and left arms differ; the dominant arm is used more. The repeatability of the temperature sensors was not tested because the heat flux sensor "vent" is only on one side and was designed to be on the right arm, closer to the armpit, which results in slower venting. No studies have been published on the reliability of the SenseWear Armband or the validity of its energy expenditure measurement. Experiments testing the validity of the SenseWear Armband measurement compared to indirect calorimetry are needed before this tool can be used for field research or publicly. 


\section{Chapter 3}

Journal Manuscript 


\begin{abstract}
An accurate energy expenditure (EE) measurement tool is needed to overcome limitations of other field EE measures. The SenseWear Armband (SWA; BodyMedia, Inc), using multiple sensors (e.g., accelerometer, heat flux) to assess EE, was designed to overcome these limitations, although no studies have been published on reliability or validity. PURPOSE: To examine the reliability and validity of the SWA during rest and exercise compared to indirect calorimetry (IC). METHODS: EE was assessed with SWA and IC in 13 males during two resting (four $10 \mathrm{~min}$ measures over 3-h) and one cycle ergometry (three 5-10 min periods within a 40 min cycle at $60 \% \mathrm{VO}_{2 \text { peak }}$ ) sessions. In a second experiment, 20 adults walked on a treadmill for $30 \mathrm{~min}(10 \mathrm{~min}$ at $3 \mathrm{mph}, 0 \%$ grade; $4 \mathrm{mph}, 0 \%$ grade; $4 \mathrm{mph}, 5 \%$ grade) while continuous IC and SWA measured EE. RESULTS: At rest, no significant differences were found between EE measurements from the SWA and IC for the individual or combined periods (SWA $1.3 \pm 0.1 \mathrm{kcal} / \mathrm{min}$, IC $1.3 \pm 0.1 \mathrm{kcal} / \mathrm{min}$ ), and the two methods were significantly correlated $(\mathrm{r}=0.76 ; \mathrm{p}<0.004)$. The SWA EE estimation was reliable when comparing the two resting visits $(r=0.93 ; \mathrm{p}<0.001)$. For the ergometer protocol, no significant differences were found between the SWA and IC measurements of EE early, mid, or late in exercise or for the total energy cost of the bout $(352.9 \pm 5.6 \mathrm{kcal}$ for SWA vs. 372.2 $\pm 16.8 \mathrm{kcal}$ for IC), although the measurements were not correlated $(\mathrm{r}=0.03-0.12)$. The SWA EE estimate of walking increased with treadmill speed but not with inclination. The SWA significantly overestimated the EE of walking with no grade $(27.4 \%$ for $3 \mathrm{mph}$, $\mathrm{p}<0.001 ; 12.6 \%$ for $4 \mathrm{mph}, \mathrm{p}<0.02$ ) and significantly underestimated $\mathrm{EE}$ on the $5 \%$ grade $(21.9 \%, \mathrm{p}<0.002)$. The SWA estimation of EE correlated with IC at each intensity $(\mathrm{r}=0.69$, 0.54, 0.47). CONCLUSIONS: The SWA provided valid and reliable estimates of EE at rest. The SWA provided similar mean estimates of EE as IC on the ergometer, however the individual error was large. The SWA overestimated the EE of flat walking and underestimated inclined walking EE.
\end{abstract}




\section{INTRODUCTION}

Physical inactivity increases the risk of developing heart disease, diabetes, colon cancer, high blood pressure, and obesity-related health risks. ${ }^{22}$ Thirty-eight percent of U.S. adults reported no participation in leisure-time physical activity in the 1997-98 Health Interview Survey $^{22}$ - a potentially modest estimate due to the impact of socially desirable responses in self-reports. ${ }^{3}$ Population-based surveys have suggested that little, if any, change has occurred in the physical activity levels of U.S. adults during the past few decades, ${ }^{8}$ however, it is commonly believed that the modern inactive lifestyles of Americans are contributing to the obesity epidemic ${ }^{16}(64.5 \%$ overweight with $30.5 \%$ obese $){ }^{7}$ One study of over 19,000 men showed that those who increased their vigorous activity over a four year period lost weight while those with sedentary lifestyles gained weight. ${ }^{5}$ Thus, it would be valuable to accurately determine baseline activity for individuals as well as change in total activity and quality of that activity over time in order to aid in determining risk of chronic disease and efficacy of various interventions. Determining the amount and pattern of physical activity done by a population or individual is problematic.

Doubly labeled water (DLW) and indirect calorimetry techniques - the gold standard measures of energy expenditure ${ }^{24}$ - are both limited in their assessment of free-living energy expenditure. Indirect calorimetry cannot assess free-living subjects as it requires a laboratory setting or a complex apparatus (e.g., portable unit), while DLW accurately assesses freeliving energy expenditure but does not provide information on the pattern or intensity of physical activity. Furthermore, the expense, time, burden for subjects and technical expertise required limit the use of these techniques by researchers, clinicians, and the public. ${ }^{2}$ Other physical activity and energy expenditure assessment tools have been developed to provide simpler, more cost-effective estimates. Physical activity records and questionnaires provide a convenient, low-cost estimation of physical activity and/or energy expenditure; however, over- or underestimation is typically significant (mean ranging from 8 to $62 \%)^{6,12,18}$ and assessment of individual physical activity is often poor, demonstrated by wide limits of agreement on bias plots or lack of correlation between the estimation and measurement tools (e.g., DLW and indirect calorimetry). ${ }^{6,18}$

To improve on the subjective nature of physical activity records and questionnaires, objective tools (e.g., heart rate monitors, pedometers, accelerometers) have been developed. 
There has been some consensus that triaxial accelerometers, which detect body acceleration in three planes, may offer the most promise in assessing physical activity energy expenditure. Laboratory investigations have found linear relationships between accelerometer output and indirect calorimetry measurements of energy expenditure during locomotion on a flat surface. ${ }^{2,13,23}$ However, estimates of energy expenditure during locomotion typically overestimate (12-49\%) the measurements made by indirect calorimetry. ${ }^{2,15,23}$ The use of triaxial accelerometers is often limited as they have been found to significantly underestimate the energy cost of walking up an inclination (8-21\%), ${ }^{11,13,15}$ cycling exercise (53-68\%), ${ }^{2,11}$ and performing activities of daily living (35-45\%). ${ }^{12,23}$ Accelerometers typically worn at the hip or waist cannot accurately assess the energy cost of non-ambulatory activity (e.g., cycling) or primarily upper body movement (e.g., gardening, washing dishes). The simultaneous use of two motion sensors (upper body and lower body), a heart rate monitor, and an individualized regression equation has provided a valid method of estimating energy expenditure during a wide range of activities. ${ }^{19,20}$ Unfortunately this method is extremely time and resource intensive, making application limited, ${ }^{20}$ and not appropriate for most investigations.

Triaxial accelerometers and other motion sensors are limited in their ability to estimate physical activity energy expenditure, but certain components of these devices make them ideal for assessing free-living activity. These devices provide an objective measure, are lightweight, do not disrupt normal activities, and are able to record data continuously over long periods of time. The SenseWear ${ }^{\mathrm{TM}}$ Armband (BodyMedia, Inc., Pittsburgh, PA) also encompasses these user-friendly components but differs from other motion sensors. The SenseWear Armband $(8.5 \times 5.3 \times 2.0 \mathrm{~cm}, 85 \mathrm{~g})$ is worn on the upper arm and collects a variety of physiologic data through multiple sensors - a two-axis accelerometer, heat flux sensor, skin temperature sensor, near-body ambient temperature sensor, and galvanic skin response sensor. The sensors gather data which can be uploaded and analyzed using computer software provided by BodyMedia, Inc. According to literature from the company, proprietary algorithms combine the multiple sensor data with individual body measurements (gender, age, height, and weight) to calculate energy expenditure. The multiple sensor array was designed to overcome the limitations of other objective energy expenditure assessment 
tools, however, no studies have been published in the scientific literature concerning the reliability or validity of this device.

The purpose of this investigation was to examine the validity of the SenseWear Armband's energy expenditure estimation during rest, cycling exercise, and walking exercise by comparing the estimation with indirect calorimetry measurements. A secondary purpose was to examine the reliability of the energy expenditure estimation of the SenseWear Armband (SWA) during rest.

\section{METHODS}

Experiment 1 (EX1). Participants. Fifteen 18-25 year-old normal weight males were recruited for the study. All participants were healthy nonsmokers and aerobically untrained ( $\leq 3 \mathrm{~d}$ of structured aerobic exercise per week). Subjects completed a health history form and provided written informed consent before participating. All procedures were approved by the Human Subjects Institutional Review Board of Virginia Tech.

Procedures. Baseline measurements of height and weight (in light clothing and no shoes) were performed on all subjects. Body fat percentage was predicted using the threesite skinfold measurement and body density equation of Jackson and Pollock. ${ }^{9}$ Aerobic fitness or peak oxygen consumption $\left(\mathrm{VO}_{2 \mathrm{pk}}\right)$ was determined using a graded exercise test on a cycle ergometer (Monark 818E Ergomed C) with continuous indirect calorimetry measurement (Sensor Medics Vmax 229, Yorba Linda, CA). Following a brief warm-up, the subject began the test at 60 watts $(1 \mathrm{kp}, 60 \mathrm{rpm})$ and intensity increased 20 watts $(1 / 3 \mathrm{kp})$ every minute until exhaustion-when the subject failed to maintain $60 \mathrm{rpm}$ after vocal encouragement.

The participants came into the laboratory on three other occasions - two resting and one submaximal exercise trials, order randomly assigned. On all occasions the subjects reported to the lab in the morning following a 12-hr fast, however upon arrival the subjects consumed $4.8 \mathrm{~g}$ of trans-10, cis-12 conjugated linoleic acid (CLA) or olive oil in capsule form as protocol for another study [Shute, M., J.W. Rankin, and J. Herbein. Acute effects of trans-10, cis-12 conjugated linoleic acid consumption on fuel use. Med Sci Sports Exerc. 35 (5, Supplement):S248, 2003]. The treatments were consumed immediately after the first resting measure and $80 \mathrm{~min}$ before the cycle ergometer exercise. Upon arrival the SWA was placed on the subjects. For resting trials, the subject relaxed in a reclining chair for at least $5 \mathrm{~min}$ 
before indirect calorimetry measurements were taken. The manufacturer recommended the SWA be worn for 30 min before any measurements were taken to allow the sensor readings to stabilize under steady physiological conditions. We planned to exclude the first measurement if it differed from the others. Four 10-min indirect calorimetry measurements were taken with the Sensor Medics Vmax 29 using a plastic hood ventilation system over a three hour period. There was an $80 \mathrm{~min}$ interval between the first and second measurement and then approximately 20 minutes between the second, third, and fourth measurements. During this time the subject reclined but remained awake, often reading. For the exercise trial, the subject relaxed for roughly 90 minutes before any measurements were taken. The cycle ergometer protocol involved pedaling at $60 \mathrm{rpm}$ at a fixed load, which represented $60 \%$ of each subject's predetermined $\mathrm{VO}_{2 \mathrm{pk}}$. Energy expenditure data was collected via a mouthpiece, while wearing a nose clip, by the Sensor Medics Vmax 229 system. This data was collected for minutes 1-10 (early), 18-23 (mid), and 30-40 (late) of the exercise bout. Upon completion of the exercise, the subject sat at rest on the cycle for 8 min while the energy expenditure of recovery was measured by the SWA and indirect calorimetry system. Instrument calibration was performed before the first and second resting measurements and before the initial cycle ergometer exercise measurement. The average $\mathrm{VO}_{2}$ and respiratory exchange ratio (RER) for each minute included in our analyses were used to compute a caloric value $(\mathrm{kcal} / \mathrm{min})$ as described by McArdle et al. ${ }^{14}$

Experiment 2 (EX2). Participants. Twenty healthy, nonsmoking adults (10 men, 10 women) aged 18-35 yr were recruited to participate in this protocol. All of the volunteers completed health history and written informed consent forms before participating. The procedures were approved by the Human Subjects Institutional Review Board of Virginia Tech.

Procedures. The subjects reported to the laboratory on only one occasion. They were instructed to refrain from consuming any food or performing any exercise for the $3 \mathrm{hr}$ before testing to ensure energy expenditure would not be elevated. Upon arrival to the laboratory, the subject's weight and height were measured (in light clothing and no shoes). This data was entered into the SWA along with the subject's age and gender. The SWA was placed on the subject and 30 min passed before any indirect calorimetry data was collected. During this 
time body fat percentage was estimated by three-site skinfold measurements and the body density formulas as described by Jackson and Pollock. ${ }^{9,10}$

The subject was connected to the Sensor Medics Vmax 229 metabolic system via a mouthpiece while wearing a nose clip; continuous $\mathrm{VO}_{2}$ measurements were collected for just over $40 \mathrm{~min}$ and used to determine energy expenditure. This included walking exercise on a treadmill for $30 \mathrm{~min}$ and a standing recovery for $10 \mathrm{~min}$. The exercise was divided into the following three levels of intensity for each $10 \mathrm{~min}$ period: $3 \mathrm{mph}$ on a $0 \%$ grade, $4 \mathrm{mph}$ on a $0 \%$ grade, and $4 \mathrm{mph}$ on a $5 \%$ grade. After completing the final stage of exercise, the treadmill speed and incline were gradually decreased and the subject stood at rest for recovery measurement. The SWA and indirect calorimetry system collected data during this post exercise recovery period.

Data Analysis. The timing of each indirect calorimetry measurement was recorded in order to synchronize with the SWA estimate. For analysis, the time of the measurement periods could not be matched precisely because the SWA time frames had to be rounded to the minute. The rounding was done in the direction of the nearest full minute, and the SWA reading was kept within the same context and exercise intensity as the indirect calorimetry measurement. Due to the time it takes for an individual to reach a physiological steady-state condition and for the indirect calorimetry measurement to reflect actual energy expenditure, the first five minutes of each 10 min measurement and the first 2 min of the 5 min measurement were not used in our analysis.

The InnerView ${ }^{\mathrm{TM}}$ Research Software (Version 1.0, build 41) provided by BodyMedia, Inc. to analyze the SWA did not use specific contextual algorithms, such as biking or walking that would be needed to get the most accurate energy expenditure estimation for the specific time periods in this study. Therefore, each SWA data file (without the indirect calorimetry data) was sent to BodyMedia, Inc. engineers along with a detailed list of the contextual information and matched time frames for which energy expenditure data were needed. BodyMedia, Inc. engineers conducted analyses on the experimental data files using their proprietary algorithms for specific contexts and returned the SWA energy expenditure estimations for each period.

Statistical Analysis. Analysis of energy expenditure was performed with two-factor repeated measures ANOVA to test for effects of time, device, and time by device interaction 
in the cycle ergometer and rest protocols and intensity, device, and their interaction in the treadmill protocol. Paired t-tests were computed to examine differences between average energy expenditure in the various contexts and total energy cost of the cycle ergometer bout as measured by indirect calorimetry and the SWA. Pearson's Product Moment correlation analyses were performed to determine associations between the SWA estimate and indirect calorimetry measurement of energy expenditure for each of the measures assessed with the ttest. The reliability of repeated measures of resting energy expenditure on different days by the SWA was examined with the Pearson's correlation. Statistical significance was defined at $\mathrm{p}<0.05$. Data are presented as means \pm SD. Bland-Altman bias plots were created to assess the agreement between the indirect calorimetry measurement and SWA estimation of energy expenditure as well as the reliability of the SWA estimate ${ }^{1}$ - limits of agreement involve the mean difference between the two measurement tools $\pm 2 \mathrm{SD}$ of the difference. Statistical analyses were completed using Statistical Packages for the Social Sciences software (SPSS, Version 11.0 for Windows, 2001, SPSS Inc., Chicago, IL) and Analyze-It ${ }^{\mathrm{TM}}$ General + Clinical Laboratory Statistics (Version 1.67, 2000, Analyze-it Software, Ltd., Leeds, England).

\section{RESULTS}

Descriptive characteristics of the subjects for EX1 and EX2 can be found in Tables 1 and 2 , respectively. Due to timing and scheduling constraints, only 13 of the 15 recruited subjects completed EX1; all 20 recruited subjects completed EX2.

EX 1: Rest and Ergometer Protocols. As there was no significant effect of time on energy expenditure measured over four times during rest, all of the four measures were averaged for analysis. The average energy expenditure value from each of the two resting trials did not differ, so these values were averaged to result in one resting energy expenditure value per subject for each of the devices. The mean resting energy expenditure estimated by the SWA $(1.3 \pm 0.1 \mathrm{kcal} / \mathrm{min})$ did not differ from the mean indirect calorimetry measurement $(1.3 \pm 0.1 \mathrm{kcal} / \mathrm{min})$. The mean measurements provided by the two devices were significantly correlated $(\mathrm{r}=0.76)$. The Bland-Altman plot (Figure 1) displays the good agreement between the two measures, with variation of less than $14 \%$ and no bias toward over- or underestimation. An approximation of the variation between the measures can be 
determined by dividing the average limit of agreement by the mean energy expenditure, and the bias can be visualized by the spread of the points around the zero axis.

The SWA estimates from the two trials (from different days) were compared to assess the reliability of this device. The SWA produced highly reliable estimates of energy expenditure at rest whether analyzed for each individual measure (range of $r=0.87$ to 0.94 ) or the average of the four measures within a trial $(\mathrm{r}=0.93)$. Good agreement between the two trials was also found with the Bland-Altman bias plot (Figure 2) further emphasizing the high reliability of the resting energy expenditure estimate. Comparably the reliability of the indirect calorimetry measured energy expenditure was lower for both the individual measures (range of $r=0.40$ to 0.55$)$ and the average of the four measures within each trial $(r=0.57)$.

The significant time by device interaction for the repeated measures ANOVA, used to analyze the 40-min cycle ergometer exercise, was reflected by an increase in the SWA estimate of energy expenditure at each time period contrasted by the stable indirect calorimetry measurement (Figure 3). Analysis with a paired t-test revealed that no significant differences existed between the SWA estimate and indirect calorimetry measurement of energy expenditure for any time period assessed — early (min 6-10), mid ( $\min 21-23)$, and late (min 36-40) (Table 3$)$. The greatest variation in means occurred early in exercise $(9.4 \pm 1.5 \mathrm{kcal} / \mathrm{min}$ for indirect calorimetry vs. $8.6 \pm 0.5 \mathrm{kcal} / \mathrm{min}$ for SWA $)$ $(\mathrm{p}>0.07)$. The SWA estimate did not correlate with the measured energy expenditure early $(\mathrm{r}$ $=0.11)$, mid $(\mathrm{r}=0.12)$, or late $(\mathrm{r}=0.03)$ in exercise. The Bland-Altman plots (Figure 4) display the wide limits of agreement between the two devices, with larger prediction errors seen for the individuals with the highest and lowest energy expenditures.

The total energy expenditure for the cycle ergometer bout was calculated using data from indirect calorimetry by multiplying the mean energy expenditure of the three measurement periods by the total duration of the exercise $(40 \mathrm{~min})$. The $\mathrm{SWA}$ collected data throughout the entire exercise session and generated a total exercise energy expenditure estimate. Similar to the individual exercise time periods, the total energy expenditure over the exercise bout did not differ significantly between the SWA estimate $(352.9 \pm 20.3 \mathrm{kcal})$ and indirect calorimetry measurement $(372.2 \pm 60.4 \mathrm{kcal})$ and the two measures were not correlated $(\mathrm{r}=$ $0.11)$. 
Each subject remained seated on the cycle ergometer for 8 min immediately following the 40 min exercise. The SWA estimated energy expenditure during recovery of exercise for $\min 4-8(8.9 \pm 1.4 \mathrm{kcal})$ was not significantly different from the indirect calorimetry measured energy expenditure $(9.7 \pm 1.9 \mathrm{kcal})(\mathrm{p}=0.10)$, however, the measures were only moderately correlated $(\mathrm{r}=0.58)$.

EX 2: Treadmill Protocol. The energy expenditure estimates by the SWA for the walking exercise at three increasing intensities $-3 \mathrm{mph}$ at $0 \%$ grade, $4 \mathrm{mph}$ at $0 \%$ grade, and $4 \mathrm{mph}$ at 5\% grade - were significantly different from the energy expenditure measurements made by indirect calorimetry. The SWA significantly overestimated the energy expenditure of walking on a flat surface at both speeds (by $38 \%$ at $3 \mathrm{mph}$, by $14 \%$ at $4 \mathrm{mph}$ ) and significantly underestimated the energy expenditure of walking on an inclination (by 22\%) (Table 4, Figure 5). The correlation coefficients between the two energy expenditure assessments were highest for the lowest exercise intensity $(r=0.69)$ and decreased with increasing exercise intensity ( $r=0.54$ for $4 \mathrm{mph}, 0 \%$ grade; $r=0.47$ for $5 \%$ grade). BlandAltman plots emphasize the consistent over- and underestimation of the SWA energy expenditure estimate of walking on a flat surface and incline, respectively, and display the wide range of agreement (Figures 6, 7, and 8). When separated by gender, the results of the analyses did not differ (data not shown).

The energy expenditure immediately following the treadmill exercise was assessed for 10 $\mathrm{min}$, and the last 5 of those minutes were used in the analyses. The total energy expenditure estimate of recovery min $6-10$ by the SWA $(6.7 \pm 1.8 \mathrm{kcal})$ did not differ significantly from the measured value $(6.6 \pm 3.2 \mathrm{kcal})$, and the two measures were highly correlated $(\mathrm{r}=0.89)$.

\section{DISCUSSION}

This study examined the validity and reliability of the SWA in estimating energy expenditure compared to simultaneous indirect calorimetry measurements. The results indicate that the SWA can provide valid and reliable estimates of resting energy expenditure. In addition, when compared to triaxial accelerometers, the SWA appears to provide a better estimate of cycling exercise energy expenditure and a similar estimate of walking energy expenditure.

The resting energy expenditure estimates were valid based upon the fact that there were no significant differences between the energy expenditure measured via indirect calorimetry 
and estimated by the SWA, the measurements were highly correlated, and the measurements had good agreement. Chen and $\operatorname{Sun}^{4}$ found accurate resting energy expenditure predictions by a triaxial accelerometer in their subjects living in a room calorimeter for $24 \mathrm{hr}-$ the accelerometer estimates did not differ from the measured values, and the two methods were highly correlated $(\mathrm{r}=0.88)$. Most accelerometer studies do not assess resting energy expenditure because they are designed to detect motion. Triaxial accelerometers calculate resting or basal metabolic rate using the subject's age, gender, height, and weight in an established prediction equation chosen by the manufacturer. ${ }^{4,12}$ The resting energy expenditure estimate made by triaxial accelerometers is only as accurate as the prediction equation used. The prediction model used to make the SWA resting energy expenditure estimate is proprietary; but according to personal communication with a BodyMedia, Inc. engineer, the model uses both sensor data and characteristics of the wearer (e.g., age, height, weight, etc.). More research is needed to determine if the incorporation of sensor data improves the resting energy expenditure estimate beyond the capabilities of established prediction equations. For example, it would be important to investigate if the SWA could detect and accurately estimate the slight elevation of energy expenditure over resting levels that occur with very light activities, as the majority of U.S. adults spend much of their time in primarily sedentary pursuits. Nichols et al. ${ }^{15}$ found the triaxial accelerometer underestimated very light activity because it could not distinguish between sedentary activities and very light activities.

The resting energy expenditure results also demonstrated that the SWA did not require a period of time (e.g., $30 \mathrm{~min}$ ) on the user, as recommended by the manufacturer, to provide an accurate estimate. The first measurement, taken approximately 5 min after the SWA was placed on the subject, did not differ from the other 3 measures. More research should be performed to determine if this is true for the estimate of physical activity energy expenditure as well.

Triaxial accelerometers have been shown to greatly underestimate the energy expenditure of nonweight-bearing activities (e.g., stationary cycling). Campbell et al. ${ }^{2}$ found the triaxial accelerometer underestimated the mean $( \pm \mathrm{SD})$ cycling exercise energy expenditure by $2.8 \pm$ $0.7 \mathrm{kcal} / \mathrm{min}$, which equated to an underestimation of $53 \%$. An even greater underestimation was found by Jakicic et al. ${ }^{11}$ — mean of $4.7 \mathrm{kcal} / \mathrm{min}$ and $68 \%$ underestimation. Compared to 
the triaxial accelerometer, the multiple sensors in the SWA give it the theoretical potential to generate an improved estimate of cycle exercise energy expenditure. In this study the SWA produced cycle ergometer exercise energy expenditure estimates that did not differ significantly from the measurements made by indirect calorimetry. This finding demonstrates the promise of the SWA estimation capabilities, however, the lack of a correlation with the indirect calorimetry measurement $(\mathrm{r}=0.03$ to 0.12$)$ and wide range of agreement (-3.8 to $3.5 \mathrm{kcal} / \mathrm{min})$ in Bland-Altman analyses imply the SWA provides a close estimate of cycling exercise energy expenditure in groups but is unsuitable for an individual estimate.

The low correlation can be partially explained by the difference in range of values generated by each device. Using the early cycle ergometer exercise time period as an example, the mean SWA energy expenditure estimate of the 13 subjects ranged from 8.0 to $9.5 \mathrm{kcal} / \mathrm{min}$ and the indirect calorimetry measurement ranged from 6.9 to $12.4 \mathrm{kcal} / \mathrm{min}$. The proprietary algorithm used to estimate the energy expenditure of cycling exercise appears to provide a similar energy expenditure for each individual for cycle ergometry in spite of differences in true energy cost. Body weight is one individual characteristic that plays a large role in energy expenditure. When energy expenditure values were expressed in relative terms $\left(\mathrm{kcal} \mathrm{kg}^{-1} \mathrm{~min}^{-1}\right)$, the range of values became similar for the SWA (0.1-0.2 $\left.\mathrm{kcal} \mathrm{kg}^{-1} \mathrm{~min}^{-1}\right)$ and indirect calorimetry $\left(0.1-0.2 \mathrm{kcal}^{1} \mathrm{~kg}^{-1} \mathrm{~min}^{-1}\right)$. Nonetheless, this factor cannot completely explain the poor correlation between energy expenditure obtained from the two devices, as the correlation coefficient only increased from $r=0.11$ to $r=0.30$ when expressed per body weight. Without knowing the contribution of each sensor and subject characteristic in the SWA cycling exercise prediction model, it is difficult to speculate how the relationship between estimated and measured energy expenditure could be improved. It appears the specific contextual model for estimating cycling energy expenditure needs to be modified in order to generate accurate individual estimates. The SWA detected the energy expenditure that occurred during cycle exercise at one intensity level; future research should assess whether the SWA can detect changes in cycle exercise intensity.

The SWA significantly overestimated the energy expenditure of walking on a horizontal surface (by 14 to 38\%) and underestimated the energy cost of walking on a 5\% grade (by $22 \%$ ). The SWA detected the increased energy expenditure that occurs with an increase in 
speed but did not detect the change in intensity that occurs with an increase in inclination (Figure 5). These results are very comparable to those found in triaxial accelerometer studies. The energy expenditure of walking on a horizontal surface was significantly overestimated by the triaxial accelerometer by 12 to $49 \%{ }^{2,15,23}$ The elevated energy expenditure that occurs when walking on an inclination was also not detected by triaxial accelerometers ${ }^{11,13}$ - underestimation ranged from 8 to $21 \%{ }^{11,15}$ The similar magnitude of over- and underestimation found in this study and triaxial accelerometer studies suggests the accelerometer in the SWA carries the most weight in the ambulatory exercise estimation model; however, the model is proprietary. The Pearson correlation coefficients were moderate for the walking exercise $(r=0.47$ to 0.69$)$. The wide limits of agreement shown by the Bland-Altman plots (Figures 6, 7, and 8) display the large error in the measurement, contributing to the moderate correlation, and emphasize the consistent over- and underestimation bias. One subject with a relatively high body mass $(121 \mathrm{~kg})$ but who was not overfat ( $\sim 15 \%$ body fat) is visually although not statistically an outlier in Figure 7 and 8 . The role of body weight in the accuracy of the SWA energy expenditure estimate of walking needs further research, data from this single subject suggests that a high body weight elicits an inferior energy expenditure estimation.

Energy expenditure is elevated above resting values following exercise, and unlike the triaxial accelerometer, ${ }^{17}$ the SWA was able to accurately estimate the energy expenditure in the period shortly following both cycling and walking exercise. The SWA estimate of recovery energy expenditure did not differ significantly from the indirect calorimetry measurement in the cycle ergometer or treadmill protocol, however, the two measures were highly correlated $(\mathrm{r}=0.89)$ following the walking exercise and were moderately correlated $(\mathrm{r}$ $=0.58$ ) following the cycle ergometry bout. This finding is intriguing and demonstrates the sensors in the SWA, other than the accelerometer, are playing a role in making improved estimates of energy expenditure during recovery from exercise.

The SWA proved to be highly reliable at estimating the energy expenditure of rest in that the mean resting energy expenditure estimates from each trial were highly correlated $(r=$ 0.93 ) and displayed good agreement. In this study the SWA resting energy expenditure estimates were the only valid estimates produced, compared to the indirect calorimetry energy expenditure measurements. It is necessary to test the reliability of the SWA in 
estimating the energy expenditure of more variable activities, such as cycling, ambulatory, and eventually lifestyle activities, and in various populations before conclusions can be made about the overall reliability of this device. The results also showed that the repeatability of the SWA resting energy expenditure estimate was stronger, as evidenced by the higher correlation coefficient, than the indirect calorimetry measured energy expenditure. Although slight variation is expected from the indirect calorimetry measurement, the SWA appears to be less sensitive to variations in resting energy expenditure.

The SWA estimates and indirect calorimetry measurements did not differ significantly for the energy expenditure of rest, cycle ergometer exercise, and following the cycling and walking exercise. The greatest variation between SWA and indirect calorimetry values occurred for the cycle ergometer early time period (min 6-10) with a trend toward significance $(\mathrm{p}<0.07)$. In order to determine whether a Type II error was committed, power analysis was performed to determine the number of subjects needed to find a significant difference between the SWA mean estimate and indirect calorimetry mean measurement for this time period. The results indicated we would need 80 subjects to have a power of 0.80 for a difference of this magnitude. The other measures that did not differ significantly would require an even greater sample size to approach significance.

Our study was not without limitations. The findings are based under the assumption that the indirect calorimetry measurements were stable and accurate-despite potential errors in this device as well. The subjects in EX1 and EX2 were healthy, young adults who are not representative of the general U.S. population. Therefore, our results are only generalizable to a similar population, emphasizing the need for more research on other populations. The SWA and indirect calorimetry measurements could not be synchronized exactly, however, it is not believed that the results were affected by this because the values used in the analyses were means of 3 to 5 min periods of physiological steady-state activity. The energy expenditure estimates provided by the SWA were generated from specific contextual models not available on the company's analysis software. At the time of this study, the software was only able to use an algorithm designed to assess daily energy expenditure. The assessment of daily energy expenditure is very important, but in some situations, the user (e.g., clinician, researcher, individual) needs to know the energy expenditure of a specific activity or time period. It is possible that future studies will be able to analyze the SWA data with improved 
software that is better able to detect specific contexts and apply a specific algorithm for the activity.

Overall, this study reveals that the SWA provides a valid and reliable estimate of resting energy expenditure. Compared to triaxial accelerometers, the SWA generates improved energy expenditure estimates of cycling exercise and similar energy expenditure estimates of walking exercise. As no other studies have been published on this device, further research is warranted before conclusions on the reliability, validity, and utility of the SWA can be made.

This evaluation of the SWA is somewhat preliminary as the device is still in stages of development. The energy expenditure estimation models and the software capabilities are being improved and updated as studies are performed. Despite the limitations of the energy expenditure estimate and analysis software, the SWA appears to provide an improved estimate of energy expenditure over triaxial accelerometers. The device is also user-friendly in terms of easy attachment/detachment, minimal discomfort, and little or no interference in activity. The cost of the SWA is relatively high (up to $\$ 995$ for the SWA and $\$ 4995$ for the analysis software) compared to accelerometers (up to $\$ 450$; year of costing 2001), ${ }^{1}$ however, the price is currently coming down. According to the manufacturer, a new version will be available for a much reduced price and the analysis software will be available on-line for a low annual fee; these changes will make the device more accessible for widespread use. The degree of difference between the SWA estimated energy expenditure and the actual or measured energy expenditure that would be considered acceptable would depend on the reason for its use-large population studies would likely accept more variation than clinical practitioners. For example the similar group estimates of cycling exercise energy expenditure provided by the SWA may provide acceptable accuracy for epidemiologists despite the large individual error. Similarly the energy expenditure of walking on a flat surface was significantly overestimated by the SWA, however, in practical terms the average absolute difference in caloric expenditure of walking for 30 min would be only approximately $25-45 \mathrm{kcal}$. This would translate to $150-270 \mathrm{kcal}$ per week overestimation of energy expended for someone following the CDC recommendation to do moderate activity on most days of the week. Using a value of $1591 \mathrm{kcal}$ per kilogram of fat, an individual would gain a kilogram every 6 to 11 weeks if they relied exclusively on the estimation from this monitor to determine energy intake. Ideally, reduction in the error of energy expenditure 
estimation for various activities to within $20 \%$ of measured values may make these tools useful for population research and individuals. More research is needed before this device is ready for widespread use; but in terms of estimation accuracy and ease of use, it has the potential to provide a feasible assessment of free-living energy expenditure. 


\section{REFERENCES}

1. Bland, J. M. and D. G. Altman. Statistical methods for assessing agreement between two methods of clinical measurement. Lancet. i:307-310, 1986.

2. Campbell, K. L., P. R. Crocker, and D. C. McKenzie. Field evaluation of energy expenditure in women using Tritrac accelerometers. Med Sci Sports Exerc. 34:16671674, 2002.

3. Chasan-Taber, L., J. B. Erickson, P. C. Nasca, S. Chasan-Taber, and P. S. Freedson. Validity and reproducibility of a physical activity questionnaire in women. Med Sci Sports Exerc. 34:987-992, 2002.

4. Chen, K. Y. and M. Sun. Improving energy expenditure estimation by using a triaxial accelerometer. J Appl Physiol. 83:2112-2122, 1997.

5. Coakley, E. H., E. B. Rimm, G. Colditz, I. Kawachi, and W. Willett. Predictors of weight change in men: results from the Health Professionals Follow-up Study. Int J Obes Relat Metab Disord. 22:89-96, 1998.

6. Conway, J. M., J. L. Seale, D. R. Jacobs, Jr., M. L. Irwin, and B. E. Ainsworth. Comparison of energy expenditure estimates from doubly labeled water, a physical activity questionnaire, and physical activity records. Am J Clin Nutr. 75:519-525, 2002.

7. Flegal, K. M., M. D. Carroll, C. L. Ogden, and C. L. Johnson. Prevalence and trends in obesity among US adults, 1999-2000. Jama. 288:1723-1727, 2002.

8. French, S. A., M. Story, and R. W. Jeffery. Environmental influences on eating and physical activity. Annu Rev Public Health. 22:309-335, 2001.

9. Jackson, A. S. and M. L. Pollock. Generalized equations for predicting body density of men. Br J Nutr. 40:497-504, 1978.

10. Jackson, A. S., M. L. Pollock, and A. Ward. Generalized equations for predicting body density of women. Med Sci Sports Exerc. 12:175-181, 1980.

11. Jakicic, J. M., C. Winters, K. Lagally, J. Ho, R. J. Robertson, and R. R. Wing. The accuracy of the TriTrac-R3D accelerometer to estimate energy expenditure. Med Sci Sports Exerc. 31:747-754, 1999. 
12. Leenders, N. Y., W. M. Sherman, H. N. Nagaraja, and C. L. Kien. Evaluation of methods to assess physical activity in free-living conditions. Med Sci Sports Exerc. 33:1233-1240, 2001.

13. Levine, J. A., P. A. Baukol, and K. R. Westerterp. Validation of the Tracmor triaxial accelerometer system for walking. Med Sci Sports Exerc. 33:1593-1597, 2001.

14. McArdle, W. D., F. I. Katch, and V. L. Katch. Exercise physiology : energy, nutrition, and human performance. Philadelphia: Lea \& Febiger, 1981, xi, 508.

15. Nichols, J. F., C. G. Morgan, J. A. Sarkin, J. F. Sallis, and K. J. Calfas. Validity, reliability, and calibration of the Tritrac accelerometer as a measure of physical activity. Med Sci Sports Exerc. 31:908-912, 1999.

16. Saris, W. H. Fit, fat and fat free: the metabolic aspects of weight control. Int J Obes Relat Metab Disord. 22 Suppl 2:S15-21, 1998.

17. Sherman, W. M., D. M. Morris, T. E. Kirby, R. A. Petosa, B. A. Smith, D. J. Frid, and N. Leenders. Evaluation of a commercial accelerometer (Tritrac-R3 D) to measure energy expenditure during ambulation. Int J Sports Med. 19:43-47, 1998.

18. Starling, R. D., D. E. Matthews, P. A. Ades, and E. T. Poehlman. Assessment of physical activity in older individuals: a doubly labeled water study. J Appl Physiol. 86:2090-2096, 1999.

19. Strath, S. J., D. R. Bassett, Jr., A. M. Swartz, and D. L. Thompson. Simultaneous heart rate-motion sensor technique to estimate energy expenditure. Med Sci Sports Exerc. 33:2118-2123, 2001.

20. Strath, S. J., D. R. Bassett, Jr., D. L. Thompson, and A. M. Swartz. Validity of the simultaneous heart rate-motion sensor technique for measuring energy expenditure. Med Sci Sports Exerc. 34:888-894, 2002.

21. Tudor-Locke, C., B. E. Ainsworth, R. W. Thompson, and C. E. Matthews. Comparison of pedometer and accelerometer measures of free-living physical activity. Med Sci Sports Exerc. 34:2045-2051, 2002.

22. U.S. Department of Health and Human Services. Physical activity fundamental to preventing disease: U.S. Department of Health and Human Services, Office of the Assistant Secretary for Planning and Evaluation, 2002. 
23. Welk, G. J., S. N. Blair, K. Wood, S. Jones, and R. W. Thompson. A comparative evaluation of three accelerometry-based physical activity monitors. Med Sci Sports Exerc. 32:S489-497, 2000.

24. Westerterp, K. R. Assessment of physical activity level in relation to obesity: current evidence and research issues. Med Sci Sports Exerc. 31:S522-525, 1999. 


\section{TABLES}

TABLE 1. Descriptive characteristics of subjects, Experiment 1.

\begin{tabular}{|lcc|}
\hline & $\begin{array}{c}\text { Value } \\
\text { Mean (SD) }\end{array}$ & Range \\
\hline Age (yr) & $20.2(1.0)$ & $19-22$ \\
Weight (kg) & $76.4(11.1)$ & $68-100$ \\
Height (cm) & $176.4(5.9)$ & $170-185$ \\
Body fat (\%) & $14.4(4.4)$ & $7.0-22.4$ \\
\hline
\end{tabular}


TABLE 2. Descriptive characteristics of subjects, Experiment 2.

\begin{tabular}{|lcc|}
\hline & $\begin{array}{c}\text { Value } \\
\text { Male (n=10) }\end{array}$ & Range \\
\hline Age (yr) & $25.2(3.2)$ & $22-31$ \\
Weight (kg) & $79.5(17.2)$ & $63-121$ \\
Height (cm) & $180.9(5.3)$ & $175-188$ \\
Body fat (\%) & $10.8(5.1)$ & $4.2-18.6$ \\
& Value & Range \\
Female (n=10) & Mean (SD) & \\
\hline Age (yr) & $25.3(3.2)$ & $22-32$ \\
Weight (kg) & $62.4(10.1)$ & $50-76$ \\
Height (cm) & $163.8(6.6)$ & $155-175$ \\
Body fat (\%) & $18.6(6.7)$ & $7.9-29.3$ \\
& & \\
\hline
\end{tabular}


Table 3. Comparison of energy expenditure in $\mathrm{kcal} / \mathrm{min}$ for constant intensity cycle ergometer exercise by IC and SWA. Mean (SD).

\begin{tabular}{|lccc|}
\hline Time Period & IC & SWA & $\begin{array}{c}\text { IC vs. SWA } \\
\text { Difference (\%) }\end{array}$ \\
\hline Early & $9.4(1.5)$ & $8.6(0.5)$ & 8.8 \\
Mid & $9.3(1.4)$ & $8.9(0.5)$ & 4.0 \\
Late & $9.2(1.8)$ & $9.1(0.6)$ & 1.3 \\
\hline
\end{tabular}

Values are means of 3 (mid) or 5 (early and late) min measurement periods.

$\mathrm{IC}=$ indirect calorimetry

SWA $=$ SenseWear Armband 
Table 4. Comparison of energy expenditure in $\mathrm{kcal} / \mathrm{min}$ for walking intensities by the SWA and IC. Mean (SD).

\begin{tabular}{|lccc|}
\hline Intensity & IC & SWA & $\begin{array}{c}\text { IC vs. SWA } \\
\text { Difference (\%) }\end{array}$ \\
\hline $3 \mathrm{mph}, 0 \%$ grade & $3.9(1.1)$ & $5.4(1.2)$ & $38.0^{* *}$ \\
$4 \mathrm{mph}, 0 \%$ grade & $5.9(1.7)$ & $6.8(1.0)$ & $14.3^{*}$ \\
$4 \mathrm{mph}, 5 \%$ grade & $8.4(2.5)$ & $6.6(1.3)$ & $21.9^{* *}$ \\
\hline
\end{tabular}

Values are means of 5 min measurement periods.

$\mathrm{IC}=$ indirect calorimetry

$\mathrm{SWA}=$ SenseWear Armband

${ }^{*} \mathrm{P}<0.05$ for comparison of devices

${ }^{* *} \mathrm{P}<0.01$ for comparison of devices 


\section{FIGURES}

Figure 1. Bland-Altman bias plot for average resting energy expenditure.

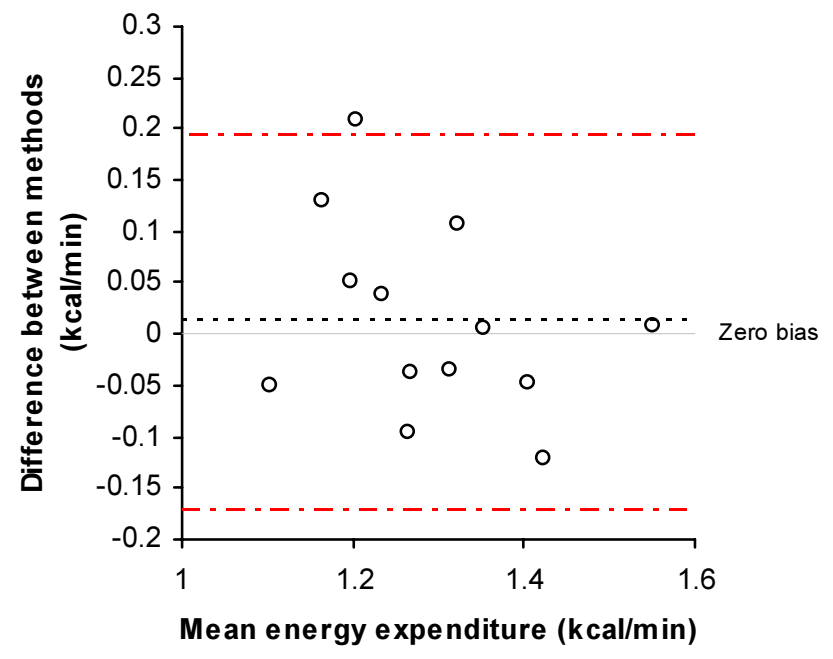

95\% Limits of Agreement $=-0.17$ to 0.20 $95 \% \mathrm{CI}=-0.04$ to 0.07 
Figure 2. Bland-Altman bias plot for SWA mean resting energy expenditure--trial 1 vs. 2.

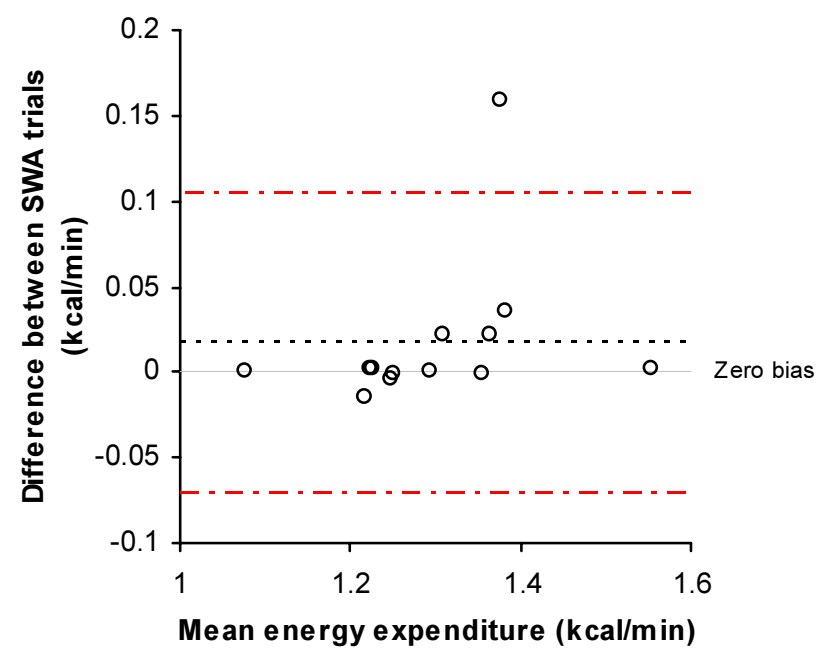

$95 \%$ Limits of Agreement $=-0.07$ to 0.10 $95 \% \mathrm{CI}=-0.01$ to 0.04 
Figure 3. Cycle ergometer mean energy expenditure measured by IC and SWA--early, mid, and late in constant intensity exercise.

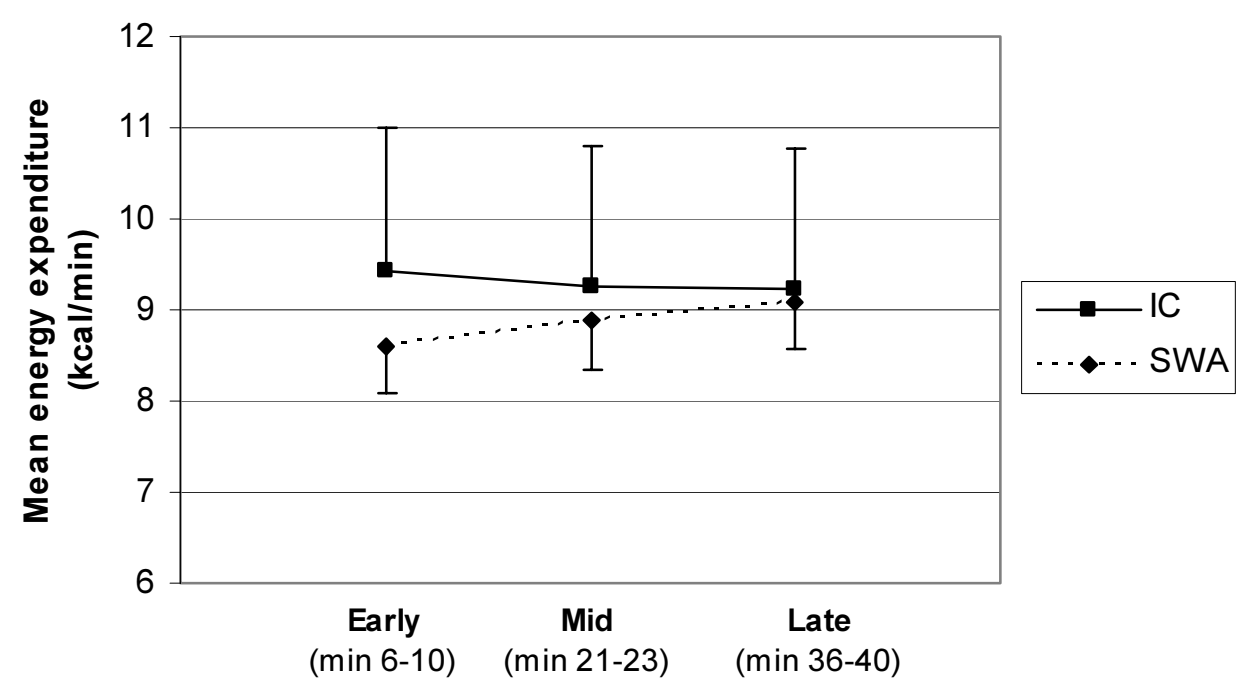

Values are means $( \pm$ SD) of 3 (mid) and 5 (early and late) min measurement periods. $\mathrm{IC}=$ indirect calorimetry

$\mathrm{SWA}=$ SenseWear Armband 
Figure 4. Bland-Altman bias plot for early (A), mid (B), and late (C) ergometer exercise energy expenditure.

A. $95 \%$ Limits of Agreement $=-3.79$ to 2.12 $95 \% \mathrm{CI}=-1.75$ to 0.08

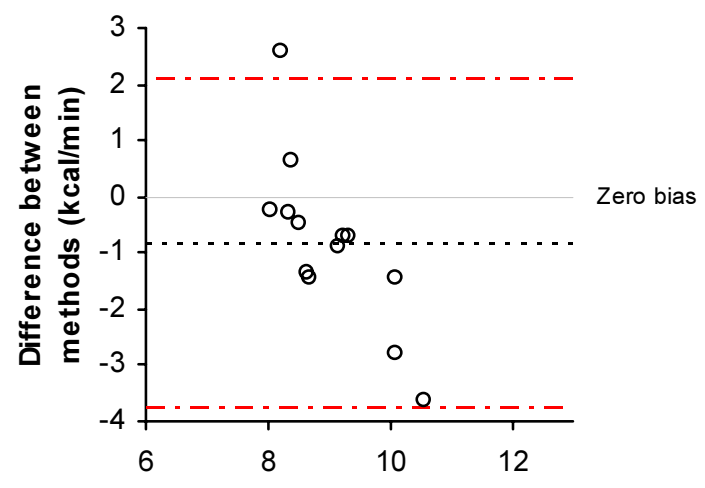

Mean energy expenditure (kcal/min)

B. $95 \%$ Limits of Agreement $=-3.26$ to 2.52 $95 \% \mathrm{CI}=-1.26$ to 0.52

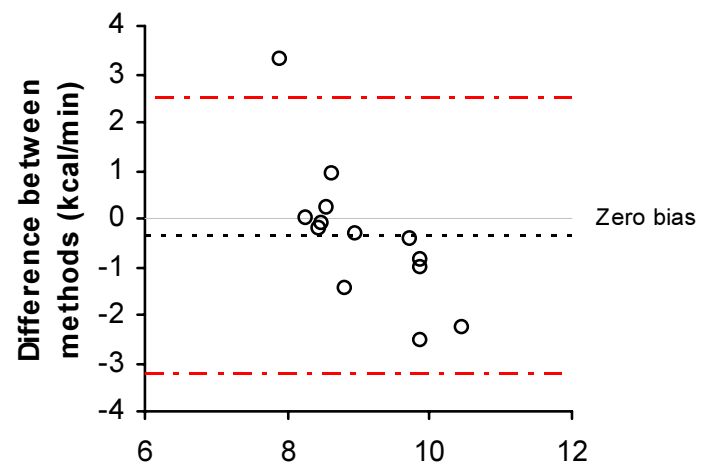

Mean energy expenditure (kcal/min)

C. $95 \%$ Limits of Agreement $=-3.68$ to 3.45 $95 \% \mathrm{CI}=-1.22$ to 0.98

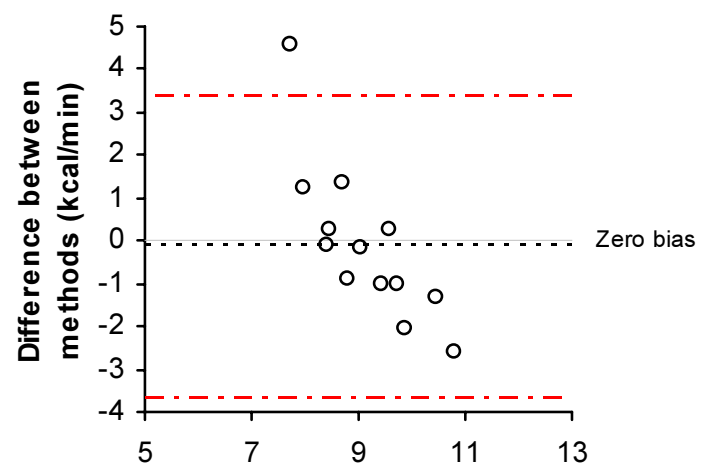

Mean energy expenditure (kcal/min) 
Figure 5. Mean walking exercise energy expenditure estimates of the SWA compared to measurements by IC.

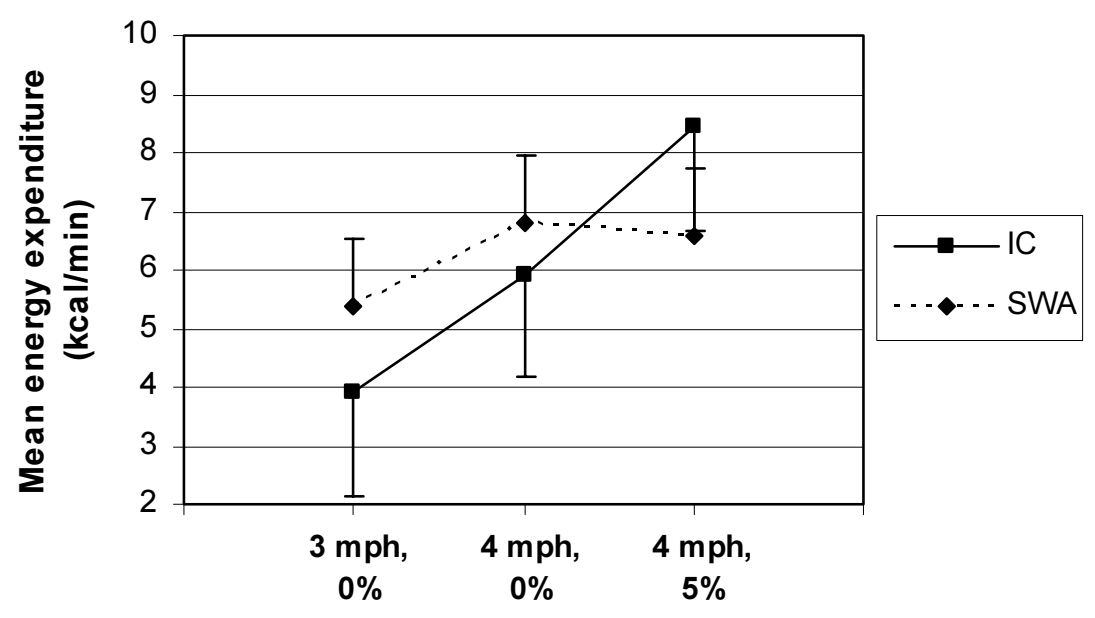

Values are means $( \pm \mathrm{SD})$ of 5 min measurement periods.

$\mathrm{IC}=$ indirect calorimetry

SWA $=$ SenseWear Armband 
Figure 6. Bland-Altman bias plot for $3 \mathrm{mph}, 0 \%$ grade walking energy expenditure.

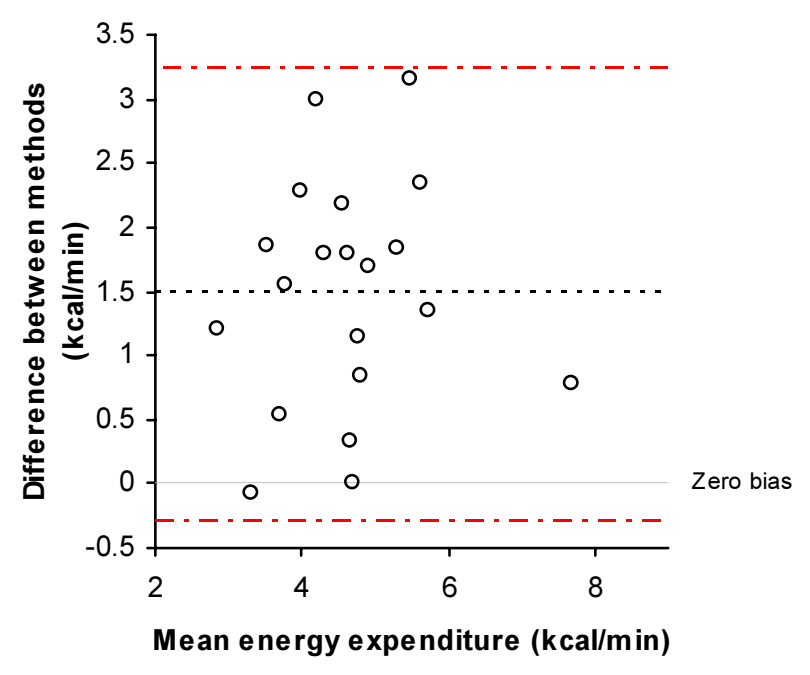

$95 \%$ Limits of Agreement $=-0.29$ to 3.25

$95 \% \mathrm{CI}=1.06$ to 1.90 
Figure 7. Bland-Altman bias plot for $4 \mathrm{mph}, 0 \%$ grade walking energy expenditure.

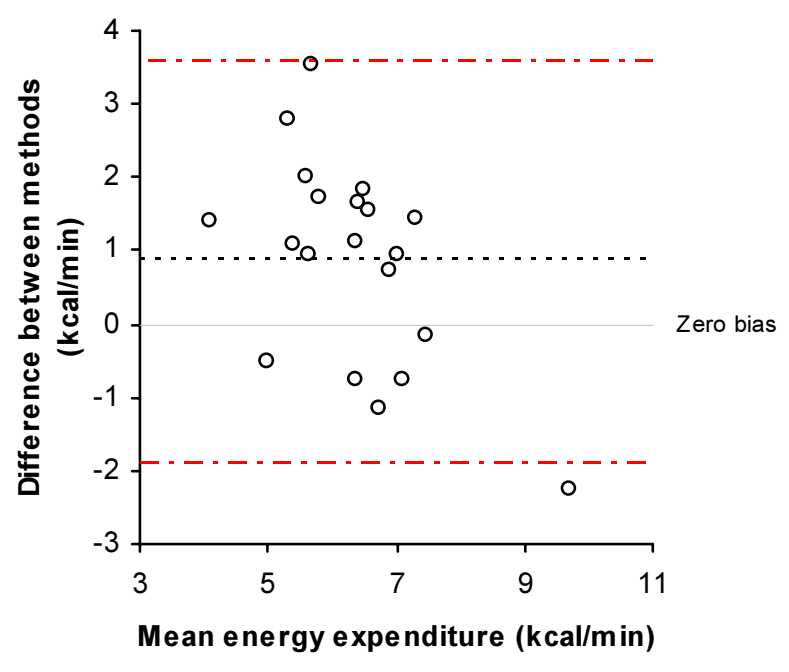

95\% Limits of Agreement $=-1.90$ to 3.62 $95 \% \mathrm{CI}=0.20$ to 1.52 
Figure 8. Bland-Altman bias plot for $4 \mathrm{mph}$, $5 \%$ grade walking energy expenditure.

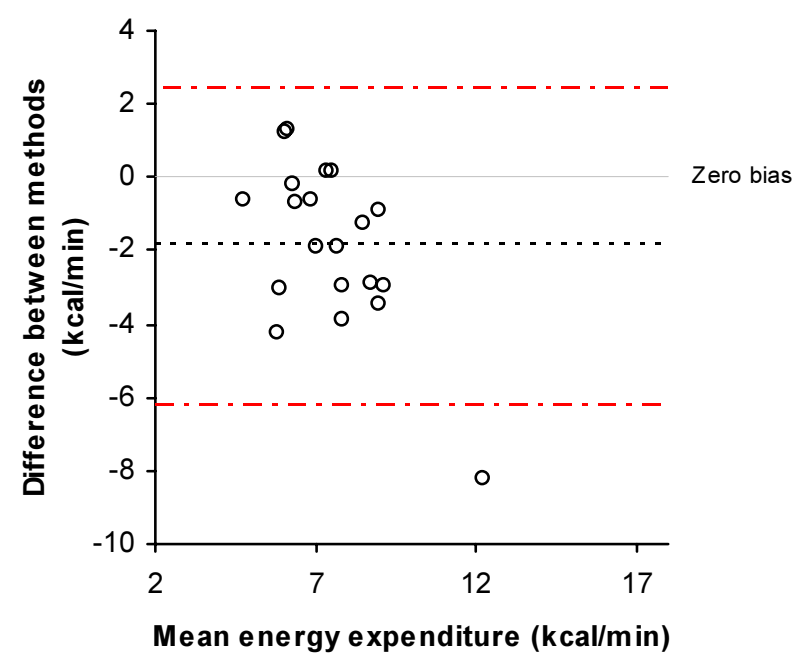

95\% Limits of Agreement $=-6.21$ to 2.52 $95 \% \mathrm{CI}=-2.89$ to -0.80 


\section{Chapter 4}

Summary and Recommendations 
Information on the free-living physical activity energy expenditure of the population, clinical patients, and individuals is routinely desired, however, quantifying daily or physical activity energy expenditure is a current challenge. Energy expenditure can be accurately measured with indirect calorimetry in a laboratory/research setting and with doubly labeled water (DLW) in a free-living environment (with some laboratory components). In spite of this, these techniques require expensive equipment and supplies and require knowledgeable technicians during the measurement period and subsequent analysis. Other tools have been developed to assess physical activity and energy expenditure outside of the laboratory that are simpler and lower in cost, such as physical activity records and recall questionnaires, pedometers, heart rate monitors, and accelerometers. Numerous limitations exist in the capabilities of these tools; therefore, an accurate energy expenditure measurement tool for use outside of the laboratory is needed to overcome these limitations. The SenseWear Armband (SWA) using multiple sensors - a two-axis accelerometer, heat flux sensor, skin temperature sensor, near-body ambient temperature sensor, galvanic skin response sensor, and optional heart rate receiver - was designed for this purpose. However, to date no studies have been published on the reliability or validity of the SWA's energy expenditure estimation.

The energy expenditure estimate of the SWA was compared to the simultaneous energy expenditure measurement made by indirect calorimetry. Both measurement devices assessed the energy expenditure of subjects at rest, during 40 min cycle ergometer exercise at $60 \%$ $\mathrm{VO}_{2 \text { peak }}$, and during treadmill walking at three intensity levels. As no studies have been published on the SWA, the results of our study have been compared to the results of studies on triaxial accelerometers. The triaxial accelerometer was chosen because there has been some consensus in the scientific literature that triaxial accelerometers offer the most promise in assessing physical activity energy expenditure.

The SWA was found to provide a valid estimation of resting energy expenditure. No significant differences were found between the individual measurements of energy expenditure or the pooled average from the SWA $(1.3 \pm 0.1 \mathrm{kcal} / \mathrm{min})$ and indirect calorimetry $(1.3 \pm 0.1 \mathrm{kcal} / \mathrm{min})$. The values obtained by the two devices were also significantly correlated $(r=0.76)$. Few triaxial accelerometer studies have investigated resting energy expenditure because accelerometers are designed to detect motion. Triaxial 
accelerometers do provide an estimate of resting energy expenditure using an established prediction equation chosen by the manufacturer. ${ }^{15,38}$ This limits the resting energy expenditure prediction ability of triaxial accelerometers, as no one equation provides accurate estimates for all individuals. The SWA also uses some form of prediction equation; but this equation differs in that it not only considers subject characteristics (gender, age, height, and weight), it also incorporates sensor data. A more diverse group of subjects needs to be studied to determine if the sensor data provides a benefit in generating an accurate resting energy expenditure estimate.

The SWA was found to be highly reliable in estimating the energy expenditure of rest. When comparing the two resting trials performed on the same subjects on different days, the SWA measurements were highly correlated $(\mathrm{r}=0.93)$. The triaxial accelerometer was also found to be highly reliable $(r=0.87-0.92)$, in a study by Nichols et al., ${ }^{54}$ when energy expenditure was assessed during a treadmill walking and jogging protocol performed on two separate days. The reliability of the SWA energy expenditure estimation needs to be evaluated for different types and intensities of activity before conclusions on the reliability of its estimate can be drawn.

In the cycle ergometer protocol, no significant differences were found between the SWA and indirect calorimetry measurements of energy expenditure early ( $\min 6-10)$, mid (min 2123 ), or late ( $\min 36-40)$ in exercise or for the total energy cost of the bout. These findings differed greatly from triaxial accelerometer results which were found to underestimate the energy expenditure of cycling by 53 to $68 \%$ compared to indirect calorimetry measurements. ${ }^{13,34}$ The SWA mean estimation was similar to the indirect calorimetry mean measurement but there was great variation between the devices' measurements. The measurements were not correlated $(r=0.03$ to 0.12$)$ and had wide limits of agreement $(-3.79$ to 3.45), demonstrating the less than satisfactory SWA estimation for individuals.

The SWA significantly overestimated the energy expenditure of treadmill walking on a horizontal surface ( $38 \%$ for $3 \mathrm{mph} ; 14 \%$ for $4 \mathrm{mph}$ ) and significantly underestimated the energy expenditure of walking on a $5 \%$ grade $(22 \%)$. The SWA energy expenditure estimate increased with speed of the treadmill but did not increase with inclination. A moderate correlation was found between the SWA estimate and indirect calorimetry measurement at each intensity (for each increasing intensity $r=0.69,0.54,0.47$ ). The over- and 
underestimation found in this study is similar to that shown in triaxial accelerometer studies. Triaxial accelerometers have been found to significantly overestimate the energy expenditure of walking on a horizontal surface by 12 to $49 \% .^{13,54,74}$ The elevated energy expenditure that occurs when walking on an inclination was also not detected by triaxial accelerometers ${ }^{34,40}$ underestimation ranged from 8 to $21 \% .^{34,54}$

Energy expenditure is elevated above resting values in the short period following exercise (oxygen debt); and unlike the triaxial accelerometer, ${ }^{63}$ the SWA was able to accurately estimate the energy expenditure following both cycling exercise (for min 4-8) and walking exercise (for min 6-10). The SWA energy expenditure estimate did not differ significantly from the indirect calorimetry measurement in the ergometer or treadmill protocol, however, the two measures were only highly correlated $(r=0.89)$ following the walking exercise. Following the ergometer exercise, the energy expenditure measures were moderately correlated $(\mathrm{r}=0.58)$.

Overall, this study revealed that the SWA provides a valid and reliable estimate of resting energy expenditure. And compared to triaxial accelerometers, the SWA appears to generate improved energy expenditure estimates of cycling exercise and similar energy expenditure estimates of walking exercise. As no other studies have been published on this device, further research is warranted before conclusions on the reliability, validity, and utility of the SWA can be made.

\section{Future Recommendations}

1) The SWA was found to provide a valid estimate of energy expenditure in a homogeneous group of subjects at rest. As little physiological changes occur at rest, the sensors most likely play a minor role in estimating energy expenditure, while a prediction equation - as used by triaxial accelerometers - most likely plays a major role. It would be important to investigate the accuracy of the SWA in estimating the energy expenditure at rest in a more diverse population, for example groups of differing age or with greater body fat percentage.

2) The energy expenditure estimation provided by the SWA for very light intensity activities should be evaluated compared to indirect calorimetry measurements. Triaxial accelerometers are unable to distinguish between sedentary and very light 
intensity activities. ${ }^{54}$ If capable of this, the utility of the SWA in estimating daily energy expenditure would be improved over triaxial accelerometers, as many Americans engage primarily in sedentary or very light intensity activities.

3) The SWA provided a reliable assessment of resting energy expenditure in subjects on two different days. It would be important to investigate whether the SWA also provides a reliable estimate of energy expenditure for other activities, such as walking, running, cycling, or stair climbing.

4) The SWA provided a similar estimate of cycling exercise energy expenditure, but the error in the estimate was large, as seen in the lack or correlation and wide limits of agreement with the Bland-Altman plots. More research on the estimation of cycling exercise energy expenditure is needed to better evaluate the source of error. For example, it would be important to investigate whether the SWA could distinguish between different cycling exercise intensity levels.

5) A HR monitor was worn by all of our subjects because the SWA contains a HR receiver to record and analyze the subject's HR response. The manufacturer did not include the HR data in the prediction models used to analyze our data because the HR monitor is an optional tool - they didn't want users to have to wear an additional device to generate energy expenditure estimations. The incorporation of HR data may improve the energy expenditure estimate derived from the SWA, as other studies have found valid estimates when an accelerometer (or two) and HR recorder were used simultaneously. ${ }^{58,65,66}$ The incorporation of HR data into the walking model may improve the estimate of walking on an inclination, as this increase in intensity was not detected by the SWA.

6) The energy expenditure estimation of more diverse activities should be evaluated. Other objective physical activity energy expenditure assessment tools cannot accurately estimate the energy expenditure of many field activities, such as washing dishes, raking leaves, stair climbing, grocery shopping, etc. The upper arm placement of the SWA may better facilitate an accurate estimation, compared to the trunk placement of most devices; however, this has not been investigated.

7) The manufacturer's armband and analysis software should be evaluated for energy expenditure estimates over long time periods. Currently the software is designed to 
provide a daily energy expenditure estimate. The validity of this estimate could be compared to the energy expenditure measurement made by a room calorimeter or the DLW process.

8) In the future, the use of software that incorporates the specific contextual information and related prediction models would need to be evaluated. The manufacturer would not be able to analyze the data of a researcher, clinician, or individual in ordinary circumstances. 


\section{Appendix A}

Detailed Description of Research Methods and Procedures 


\section{Experiment 1 (EX1)}

The design of this experiment (e.g., subject description, resting and exercise protocol) was set up in this way because it was a companion study to another project [Shute, M., J.W. Rankin, and J. Herbein. Acute effects of trans-10, cis-12 conjugated linoleic acid consumption on fuel. Med Sci Sports Exerc. 35 (5, Supplement): S248, 2003.].

\section{Subject Selection and Screening}

Fifteen 18-25 year-old normal weight, aerobically unfit ( $\leq 3$ days of structured aerobic exercise per week) males were recruited. Fliers were hung in numerous buildings on the Virginia Tech campus, clubs and organizations were contacted via E-mail and spoken to if interested, and large undergraduate classrooms were also spoken to. The interested men attended one of two informational sessions where they were informed of the details of the study and potential risks, had their questions answered, and completed health history and informed consent forms (Appendix E). The health history form screened for risk factors that would normally exclude them from physical activity participation, including conditions such as orthopedic limitations or injuries, epilepsy or seizures, cardiovascular diseases, or other restrictions. Due to time and scheduling constraints only 13 of the 15 recruited subjects completed the study.

\section{Subject Pre-testing}

Baseline measurements were performed on all subjects including weight in light clothing without shoes on a balance-beam scale, height in bare feet with a stadiometer, and body fat percentage. Body fat percentage was determined by skinfold measurement at the abdomen, chest, and thigh using the body density formula by Jackson and Pollock. ${ }^{29}$ Three measurements were taken from each site and averaged. The same trained technician performed all measurements.

Aerobic fitness $\left(\mathrm{VO}_{2 \mathrm{pk}}\right)$ was determined using a graded exercise test on a cycle ergometer (Monark 818E Ergomed C), while continuous indirect calorimetry was performed using the Sensor Medics Vmax 229 metabolic system (Yorba Linda, CA). The aerobic fitness test involved a warm-up of 2 minutes at 30 watts $(1 / 2 \mathrm{kp}, 60 \mathrm{rpm})$ and 2 minutes at 60 watts (1 $\mathrm{kp}, 60 \mathrm{rpm})$. The exercise test began at 60 watts $(1 \mathrm{kp}, 60 \mathrm{rpm})$ and increased 20 watts $(1 / 3$ $\mathrm{kp}$ ) every minute until exhaustion — when the subject failed to maintain $60 \mathrm{rpm}$ after vocal encouragement. 


\section{Experimental Trials}

Subjects came into the laboratory on four other occasions. Two of these sessions were resting and two submaximal exercise, order randomly assigned. Prior to the first morning visit each subject recorded his dietary intake over the previous $24 \mathrm{hr}$. The subjects were encouraged to consume a similar diet the day before each of their next three visits. On all four occasions the subjects reported to their designated lab (Wallace Hall for resting and War Memorial Hall for exercise) in the morning following a 12-hr fast-without having taken any medications or supplements, used any tobacco products, or performed any exercise. The subject's height and weight (measured in pre-test), gender, and age were programmed into the SWA the night before each visit.

Note that subjects ingested a placebo (olive oil) or $4.8 \mathrm{~g}$ of 10,12 conjugated linoleic acid (CLA) capsules prior to each of the resting and exercise trials (timing explained later). This was part of the concurrent study being conducted on these subjects and was not expected to influence our data.

\section{Resting Trials}

Upon reporting to the lab in the morning, the SWA and Polar heart rate (HR) monitor were placed on the subject. The SWA and HR monitor remained on the subject collecting data for the entire visit. The subject then relaxed in a reclining chair for approximately five minutes before resting metabolic rate (RMR; $\mathrm{kcal} / \mathrm{min}$ ) was measured via indirect calorimetry using a plastic hood connected to the Sensor Medics Vmax 29 metabolic system (Yorba Linda, CA) for at least $10 \mathrm{~min}$.

Following this initial measurement the subject consumed either the placebo or CLA capsules with 8 ounces of water. The subject relaxed in the recliner while reading or studying for the next $80 \mathrm{~min}$. Then, three more indirect calorimetry measurements were taken for $10 \mathrm{~min}$ each, separated by $20 \mathrm{~min}$.

Cycle Ergometry Trials

On two other mornings each subject reported to the laboratory in War Memorial Hall where the SWA and Polar HR monitor were placed on the subject. The subject ingested the capsules (placebo or CLA) with water and relaxed for $\sim 70 \mathrm{~min}$.

After 70 min of relaxing, the subject was positioned on the stationary cycle ergometer, and the indirect calorimetry head-gear, mouthpiece, and nose clip were fitted to the subject. 
The subject sat on the cycle ergometer at rest for 10 min, while the Sensor Medics Vmax 229 collected energy expenditure data. Immediately following the 10-min baseline measurement, the subject began pedaling at $60 \mathrm{rpm}$ while the resistance was increased gradually over 2 to 3 minutes until the subject was cycling at an intensity of $\sim 60 \%$ of his predetermined $\mathrm{VO}_{2 \mathrm{pk}}$. The subject cycled at this intensity for $40 \mathrm{~min}$. Energy expenditure data was collected via indirect calorimetry for minutes $1-10,18-23$, and $30-40$ of the exercise duration. The mouthpiece was removed and the metabolic computer paused during the 8-and 7-min gaps in collection times. Upon completion of the exercise the subject sat at rest on the cycle for 8 min, while indirect calorimetry continued collecting energy expenditure data of the post exercise energy expenditure.

\section{Experiment 2 (EX2)}

\section{Subject Selection and Screening}

Twenty healthy men and women, aged 18-35 yr were recruited at Virginia Tech through mass E-mail and personal communication. Interested individuals completed health history and informed consent forms (Appendix E). The health history form screened the individuals for risk factors that would normally exclude them from physical activity participation, including conditions such as orthopedic limitations or injuries, epilepsy or seizures, cardiovascular diseases, or other restrictions. The qualified subjects were thoroughly informed on the details of the study and potential risks.

\section{Experimental Trial}

The subjects reported to the laboratory in War Memorial Hall on only one occasion, after having refrained from consuming any food or performing any exercise for the previous 3 hours. Immediately the subject's weight was measured in light clothing without shoes on a balance beam scale, and height was measured in bare feet with a stadiometer. This data was programmed into the SWA along with the subject's age and gender. The SWA and Polar HR monitor were then placed on the subject, and a full $30 \mathrm{~min}$ passed before any indirect calorimetry data was collected. This delay was recommended by the manufacturer to allow the SWA sensor readings to stabilize under steady physiological conditions.

During this time the subject relaxed while reading or working on a computer and their body fat percentage was determined by skinfold measurement. Skinfold thickness was measured at the abdomen, chest, and thigh for the men and at the suprailiac, thigh, and 
triceps for the women; and body fat percentage was calculated using the body density formulas devised by Jackson and Pollock..$^{29,30}$ The same trained technician performed all of the measurements.

After the SWA had been on the subject for at least $30 \mathrm{~min}$, the subject was connected to the Sensor Medics Vmax 229 metabolic system via a mouthpiece, and continuous energy expenditure data was collected for just over $50 \mathrm{~min}$. The subject stood at rest for $10 \mathrm{~min}$ to provide a baseline measurement and then walked on a treadmill for the next $30 \mathrm{~min}$. The exercise was divided into the following three levels of intensity for each 10 min period: 3 mph on a $0 \%$ grade, $4 \mathrm{mph}$ on a $0 \%$ grade, and $4 \mathrm{mph}$ on a $5 \%$ grade. After completing the final stage of exercise, the treadmill speed and incline were gradually decreased to a stop and the subject stepped off the belt. The subject then stood at rest for a final $10 \mathrm{~min}$; the SWA and indirect calorimetry system collected data on the post exercise energy expenditure.

\section{Matching of EE Measurement Time Periods}

The SWA was worn by the subjects for the entirety of the experimental trial visits, while indirect calorimetry measurements were either not continuous (EX1) or not taken during the entire visit (EX1 and EX2). The analysis of the validity of the SWA estimate compared to indirect calorimetry measurement required a system to match the desired indirect calorimetry energy expenditure measurement (usually a 5-min period) to the same time period of energy expenditure data collected by the SWA.

When each subject's demographic information (height, weight, age, and gender) was programmed into the SWA, either the night before or the day of the experimental trial, the internal clock on the computer used in programming the SWA was set to match the researcher's digital watch (or the watch was set to match the computer). The internal clock in the SWA was reset to match the computer's internal clock and thus the researcher's watch. Whenever an indirect calorimetry measurement began, the intensity changed, or the indirect calorimetry measurement ended, the time on the researcher's watch was recorded on a data sheet (Appendix F) next to the event that occurred. 
Example data sheet entry:

\begin{tabular}{|c|c|l|}
\hline SWA time & IC time & Description \\
\hline $10: 32.18$ & 0 & Sitting at rest \\
\hline $10: 42.20$ & $10 / 0$ & End rest / start exercise \\
\hline $10: 52.20$ & 10 & IC out \\
\hline $11: 00.20$ & 18 & IC in \\
\hline
\end{tabular}

The time of the measurement periods could not be matched between indirect calorimetry and the SWA precisely because the SWA time frames had to be rounded to the minute. For example, if the indirect calorimetry time frame of interest began at 8:47.25 on the SWA "clock," the SWA time frame would have to be rounded down to 8:47.00 or up to 8:48.00 to be analyzed. The rounding was most often done in the direction of the nearest full minute to minimize the time frame difference, but when the time was near or on the half minute, the rounding direction varied. The decision to round up or down was based on the time frame in question and was made by the researcher to keep the SWA reading within the same context and exercise intensity as the indirect calorimetry measurement.

\section{Indirect Calorimetry EE Determination}

Oxygen consumption was measured by the metabolic carts at 20 -sec intervals, and the mean of the three intervals in each minute was used for data analysis. Due to the time it takes for an individual to reach a physiological steady-state condition and for the indirect calorimetry measurement to reflect actual energy expenditure, the first 5 min of each 10-min measurement were not used in our analysis. This applied to all four resting measurements, the first (min 1-10) and last (min 30-40) cycling exercise measurements, and the five 10-min phases of the treadmill protocol (baseline, walking at three intensities, and recovery). One indirect calorimetry measurement performed during the middle of the 40 -min cycling exercise was only taken for $5 \mathrm{~min}$ ( $\min 18-23$ ). The first two minutes of this measurement were not included in the data analysis so that any changes in energy expenditure due to the subject getting re-adjusted to the mouthpiece or the dead air in the tubing were excluded. 
Instrument calibration was performed before the first and second resting measurements and the initial cycle ergometer and treadmill exercise measurements. The average $\mathrm{VO}_{2}$ and respiratory exchange ratio (RER) for each minute included in our analyses were used to compute a caloric value $(\mathrm{kcal} / \mathrm{min})$ as described by McArdle et al.: ${ }^{48}$

$\mathrm{EE}(\mathrm{kcal} / \mathrm{min})=\mathrm{VO}_{2} \mathrm{~L} / \mathrm{min} \times$ caloric equivalent per liter of $\mathrm{O}_{2}$ at the given steady-rate non-protein RQ

\section{SenseWear Armband EE Determination}

The InnerView ${ }^{\mathrm{TM}}$ Research Software (Version 1.0, build 41) currently provided by BodyMedia, Inc. to analyze the SWA output uses a general algorithm model that smoothes over the energy expenditure assessment to provide a daily average. The software does not use specific contextual algorithms, such as biking or walking that would be needed to get the most accurate energy expenditure estimation for the specific time periods in this study. We had the InnerView software but the data analysis provided was unacceptable due to the lack of contextual detection and use of specific algorithms. The specific contextual models have been developed by BodyMedia, Inc. but a software analysis program for use outside of BodyMedia, Inc. is not available. Therefore, each data file (without the indirect calorimetry data) was sent to BodyMedia, Inc. engineers electronically along with a detailed list of contextual information and matched time frames for which energy expenditure data was needed.

Example of file analysis request:

$\begin{array}{llll}15 \# 4.11 .02 & \text { resting } & 9: 13-9: 18 & (5 \mathrm{~min}) \\ & \text { biking } & 9: 18-9: 58 & (40 \mathrm{~min}) \\ & \text { biking } & 9: 23-9: 28 & (5 \mathrm{~min}) \\ & \text { biking } & 9: 38-9: 41 & (3 \mathrm{~min}) \\ & \text { biking } & 9: 53-9: 58 & (5 \mathrm{~min}) \\ & \text { resting } & 10: 01-10: 06 & (5 \mathrm{~min})\end{array}$

BodyMedia, Inc. engineers conducted analyses on the experimental data files and returned the SWA energy expenditure estimations for each time period in a report. 
Appendix B

Raw Data Tables 
Table 5. Experiment 1 Individual Subject Demographics.

\begin{tabular}{|ccccc|}
\hline Subject \# & Age (years) & Height (in) & Weight $(\mathrm{kg})$ & Body Fat $(\%)$ \\
1 & & & & \\
2 & 21 & 71 & 91.4 & 22.4 \\
3 & 21 & 67 & 73.6 & 15.3 \\
4 & 22 & 67 & 78.6 & 16.9 \\
5 & 20 & 71 & 69.5 & 9.8 \\
6 & 20 & 70 & 80.5 & 12.6 \\
7 & 19 & 67 & 68.2 & 11.2 \\
8 & 21 & 67 & 70.9 & 10.3 \\
9 & 21 & 68 & 70.5 & 14.7 \\
10 & 21 & 73 & 80.5 & 17.1 \\
11 & 19 & 72 & 100.0 & 21.6 \\
15 & 20 & 71 & 75.0 & 14.7 \\
16 & 19 & 72 & 79.5 & 13.8 \\
Mean & 19 & 67 & 55.0 & 7 \\
Std Dev & $\mathbf{2 0 . 2 3}$ & $\mathbf{6 9 . 4 6}$ & $\mathbf{7 6 . 4 0}$ & $\mathbf{1 4 . 4 2}$ \\
& 1.013 & 2.332 & 11.091 & 4.444 \\
\hline
\end{tabular}


Table 6. Experiment 2 Individual Subject Demographics.

\begin{tabular}{|cccccc|}
\hline Subject \# & Gender & Age (years) & Height (in) & Weight $(\mathrm{kg})$ & Body Fat $(\%)$ \\
101 & male & 31 & 72 & 67.3 & 8.2 \\
102 & male & 24 & 70 & 85.0 & 17.2 \\
103 & male & 25 & 74 & 88.2 & 12.8 \\
104 & male & 23 & 70 & 62.7 & 6.1 \\
105 & male & 31 & 74 & 83.2 & 11.9 \\
106 & male & 24 & 69 & 81.4 & 18.6 \\
113 & male & 23 & 70 & 69.5 & 6 \\
114 & male & 25 & 69 & 64.5 & 7.4 \\
118 & male & 24 & 74 & 120.9 & 15.4 \\
119 & male & 22 & 70 & 71.8 & 4.2 \\
Mean & & $\mathbf{2 5 . 2}$ & $\mathbf{7 1 . 2}$ & $\mathbf{7 9 . 5}$ & $\mathbf{1 0 . 8}$ \\
Std Dev & & 3.190 & 2.098 & 80.808 & 5.112 \\
\hline & & & & \\
107 & female & 29 & 61 & 56.8 & 12.9 \\
108 & female & 28 & 64 & 69.1 & 23.3 \\
109 & female & 32 & 64 & 58.6 & 18.3 \\
110 & female & 22 & 63 & 50.0 & 10 \\
111 & female & 24 & 65 & 49.1 & 7.9 \\
112 & female & 23 & 65 & 75.0 & 29.3 \\
115 & female & 24 & 65 & 55.9 & 21.4 \\
116 & female & 24 & 61 & 60.0 & 19.4 \\
117 & female & 23 & 69 & 73.2 & 24.6 \\
120 & female & 24 & 68 & 75.9 & 18.7 \\
Mean & & $\mathbf{2 5 . 3}$ & $\mathbf{6 4 . 5}$ & $\mathbf{6 2 . 4}$ & $\mathbf{1 8 . 6}$ \\
Std Dev & & 3.234 & 2.593 & 10.145 & 6.684 \\
\hline
\end{tabular}


Table 7. Indirect Calorimetry and SWA Resting Energy Expenditure Measurements (kcal/min): CLA* Trial.

\begin{tabular}{|c|c|c|c|c|c|}
\hline $\begin{array}{c}\text { Subject } \\
\#\end{array}$ & $\begin{array}{c}\text { IC } \\
\text { Measure } 1\end{array}$ & $\begin{array}{c}\text { IC } \\
\text { Measure } 2\end{array}$ & $\begin{array}{c}\text { IC } \\
\text { Measure } 3\end{array}$ & $\begin{array}{c}\text { IC } \\
\text { Measure } 4\end{array}$ & $\begin{array}{c}\text { IC } \\
\text { Average }\end{array}$ \\
\hline 1 & 1.318 & 1.610 & 1.446 & 1.501 & 1.469 \\
\hline 2 & 1.256 & 1.254 & 1.161 & 1.184 & 1.214 \\
\hline 3 & 1.274 & 1.435 & 1.502 & 1.537 & 1.437 \\
\hline 4 & 1.248 & 1.240 & 1.298 & 1.319 & 1.277 \\
\hline 5 & 1.763 & 1.431 & 1.371 & 1.497 & 1.515 \\
\hline 6 & 1.305 & 1.468 & 1.392 & 1.331 & 1.374 \\
\hline 7 & 1.231 & 1.116 & 1.172 & 1.335 & 1.214 \\
\hline 8 & 1.032 & 1.010 & 1.095 & 1.017 & 1.039 \\
\hline 9 & 1.340 & 1.644 & 1.377 & 1.358 & 1.430 \\
\hline 10 & 1.611 & 1.521 & 1.511 & 1.790 & 1.608 \\
\hline 11 & 0.939 & 1.184 & 1.092 & 1.136 & 1.088 \\
\hline 15 & 1.425 & 1.357 & 1.268 & 1.255 & 1.326 \\
\hline 16 & 1.007 & 1.045 & 1.058 & 1.137 & 1.062 \\
\hline Mean & 1.288 & 1.332 & 1.288 & 1.338 & 1.312 \\
\hline Std Dev & 0.228 & 0.208 & 0.159 & 0.206 & 0.182 \\
\hline $\begin{array}{c}\text { Subject } \\
\#\end{array}$ & $\begin{array}{c}\text { SWA } \\
\text { Measure } 1\end{array}$ & $\begin{array}{c}\text { SWA } \\
\text { Measure } 2\end{array}$ & $\begin{array}{c}\text { SWA } \\
\text { Measure } 3\end{array}$ & $\begin{array}{c}\text { SWA } \\
\text { Measure } 4\end{array}$ & $\begin{array}{c}\text { SWA } \\
\text { Average }\end{array}$ \\
\hline 1 & 1.455 & 1.457 & 1.456 & 1.454 & 1.456 \\
\hline 2 & 1.247 & 1.246 & 1.245 & 1.249 & 1.247 \\
\hline 3 & 1.295 & 1.295 & 1.294 & 1.295 & 1.294 \\
\hline 4 & 1.251 & 1.253 & 1.252 & 1.251 & 1.252 \\
\hline 5 & 1.349 & 1.448 & 1.349 & 1.347 & 1.373 \\
\hline 6 & 1.209 & 1.210 & 1.208 & 1.208 & 1.209 \\
\hline 7 & 1.226 & 1.224 & 1.227 & 1.223 & 1.225 \\
\hline 8 & 1.229 & 1.229 & 1.229 & 1.230 & 1.229 \\
\hline 9 & 1.505 & 1.365 & 1.364 & 1.367 & 1.400 \\
\hline 10 & 1.554 & 1.554 & 1.555 & 1.554 & 1.554 \\
\hline 11 & 1.388 & 1.300 & 1.298 & 1.298 & 1.321 \\
\hline 15 & 1.355 & 1.357 & 1.356 & 1.356 & 1.356 \\
\hline 16 & 1.078 & 1.080 & 1.076 & 1.077 & 1.078 \\
\hline Mean & 1.318 & 1.309 & 1.301 & 1.301 & 1.307 \\
\hline Std Dev & 0.133 & 0.126 & 0.120 & 0.120 & 0.123 \\
\hline
\end{tabular}

Values are means of 5 min measurement periods.

${ }^{*}$ CLA (conjugated linoleic acid) treatment used for a companion study. 
Table 8. Indirect Calorimetry and SWA Resting Energy Expenditure Measurements (kcal/min): Placebo* Trial.

\begin{tabular}{|c|c|c|c|c|c|}
\hline $\begin{array}{c}\text { Subject } \\
\#\end{array}$ & $\begin{array}{c}\text { IC } \\
\text { Measure 1 }\end{array}$ & $\begin{array}{c}\text { IC } \\
\text { Measure } 2\end{array}$ & $\begin{array}{c}\text { IC } \\
\text { Measure } 3 \\
\end{array}$ & $\begin{array}{c}\text { IC } \\
\text { Measure } 4 \\
\end{array}$ & $\begin{array}{c}\text { IC } \\
\text { Average }\end{array}$ \\
\hline 1 & 1.014 & 1.045 & 1.091 & 1.119 & 1.067 \\
\hline 2 & 1.382 & 1.529 & 1.248 & 1.270 & 1.357 \\
\hline 3 & 1.324 & 1.267 & 1.105 & 1.199 & 1.224 \\
\hline 4 & 1.062 & 1.204 & 1.177 & 1.163 & 1.152 \\
\hline 5 & 1.410 & 1.394 & 1.403 & 1.602 & 1.453 \\
\hline 6 & 1.174 & 1.228 & 1.325 & 1.275 & 1.250 \\
\hline 7 & 1.151 & 1.220 & 1.001 & 1.159 & 1.133 \\
\hline 8 & 1.131 & 1.074 & 1.195 & 1.233 & 1.158 \\
\hline 9 & 1.397 & 1.497 & 1.435 & 1.389 & 1.430 \\
\hline 10 & 1.380 & 1.360 & 1.522 & 1.679 & 1.485 \\
\hline 11 & 1.102 & 1.048 & 1.171 & 1.130 & 1.113 \\
\hline 15 & 1.388 & 1.336 & 1.338 & 1.450 & 1.378 \\
\hline 16 & 1.279 & 1.145 & 1.150 & 1.203 & 1.194 \\
\hline Mean & 1.246 & 1.258 & 1.243 & 1.298 & 1.261 \\
\hline Std Dev & 0.144 & 0.160 & 0.152 & 0.181 & 0.142 \\
\hline $\begin{array}{c}\text { Subject } \\
\#\end{array}$ & $\begin{array}{c}\text { SWA } \\
\text { Measure } 1\end{array}$ & $\begin{array}{c}\text { SWA } \\
\text { Measure } 2\end{array}$ & $\begin{array}{c}\text { SWA } \\
\text { Measure } 3\end{array}$ & $\begin{array}{c}\text { SWA } \\
\text { Measure } 4\end{array}$ & $\begin{array}{c}\text { SWA } \\
\text { Average }\end{array}$ \\
\hline 1 & 1.353 & 1.261 & 1.304 & 1.267 & 1.296 \\
\hline 2 & 1.252 & 1.250 & 1.253 & 1.251 & 1.252 \\
\hline 3 & 1.293 & 1.295 & 1.294 & 1.295 & 1.294 \\
\hline 4 & 1.251 & 1.254 & 1.253 & 1.253 & 1.253 \\
\hline 5 & 1.348 & 1.349 & 1.348 & 1.364 & 1.352 \\
\hline 6 & 1.210 & 1.269 & 1.209 & 1.206 & 1.224 \\
\hline 7 & 1.224 & 1.224 & 1.224 & 1.223 & 1.224 \\
\hline 8 & 1.228 & 1.226 & 1.228 & 1.224 & 1.226 \\
\hline 9 & 1.365 & 1.365 & 1.365 & 1.362 & 1.364 \\
\hline 10 & 1.552 & 1.553 & 1.554 & 1.554 & 1.553 \\
\hline 11 & 1.298 & 1.298 & 1.299 & 1.302 & 1.299 \\
\hline 15 & 1.356 & 1.356 & 1.358 & 1.357 & 1.357 \\
\hline 16 & 1.078 & 1.078 & 1.076 & 1.076 & 1.077 \\
\hline Mean & 1.293 & 1.291 & 1.290 & 1.287 & 1.290 \\
\hline Std Dev & 0.111 & 0.108 & 0.111 & 0.112 & 0.110 \\
\hline
\end{tabular}

Values are means of 5 min measurement periods.

*Placebo was olive oil treatment used for a companion study. 
Table 9. Cycle Ergometer Indirect Calorimetry and SWA Energy Expenditure Measurements (kcal/min): CLA* Trial.

\begin{tabular}{|ccccccc|}
\hline Subject \# & $\begin{array}{c}\text { IC } \\
\text { Early }\end{array}$ & $\begin{array}{c}\text { SWA } \\
\text { Early }\end{array}$ & $\begin{array}{c}\text { IC } \\
\text { Mid }\end{array}$ & $\begin{array}{c}\text { SWA } \\
\text { Mid }\end{array}$ & $\begin{array}{c}\text { IC } \\
\text { Late }\end{array}$ & $\begin{array}{c}\text { SWA } \\
\text { Late }\end{array}$ \\
\hline & & & & & & \\
1 & 9.544 & 9.174 & 10.188 & 9.481 & 9.510 & 9.260 \\
2 & 9.769 & 8.408 & 9.441 & 8.595 & 10.279 & 8.836 \\
3 & 11.616 & 8.543 & 11.452 & 8.551 & 10.864 & 8.508 \\
4 & 9.821 & 8.503 & 8.868 & 8.660 & 9.346 & 8.976 \\
5 & 10.185 & 9.355 & 9.673 & 9.159 & 9.408 & 9.525 \\
6 & 8.123 & 8.438 & 8.794 & 8.922 & & \\
7 & 7.394 & 7.687 & 7.687 & 7.780 & 7.715 & \\
8 & 8.193 & 8.135 & 8.814 & 8.782 & 8.462 & 8.388 \\
9 & 9.859 & 7.696 & 10.477 & 8.068 & 10.458 & 8.295 \\
10 & 10.685 & 9.295 & 10.671 & 10.024 & 11.047 & 10.253 \\
11 & 9.264 & 8.770 & 9.331 & 9.194 & 9.667 & 9.643 \\
15 & 9.958 & 8.452 & 10.268 & 9.079 & 10.334 & 9.481 \\
16 & 6.405 & 7.664 & 5.503 & 7.798 & 5.619 & 7.957 \\
Mean & $\mathbf{9 . 2 9 4}$ & $\mathbf{8 . 4 7 1}$ & $\mathbf{9 . 3 2 0}$ & $\mathbf{8 . 7 7 6}$ & $\mathbf{9 . 0 4 3}$ & $\mathbf{9 . 0 0 3}$ \\
Std Dev & 1.414 & 0.580 & 1.513 & 0.648 & 1.910 & 0.914 \\
\hline
\end{tabular}

Values are means of 3 (mid) and 5 (early and late) min measurement periods.

${ }^{*} \mathrm{CLA}$ (conjugated linoleic acid) treatment used for a companion study.

This data was not included in analysis as work intensity was not held constant compared to the placebo trial. 
Table 10. Cycle Ergometer Indirect Calorimetry and SWA Energy Expenditure Measurements (kcal/min): Placebo* Trial.

\begin{tabular}{|ccccccc|}
\hline Subject \# & $\begin{array}{c}\text { IC } \\
\text { Early }\end{array}$ & $\begin{array}{c}\text { SWA } \\
\text { Early }\end{array}$ & $\begin{array}{c}\text { IC } \\
\text { Mid }\end{array}$ & $\begin{array}{c}\text { SWA } \\
\text { Mid }\end{array}$ & $\begin{array}{c}\text { IC } \\
\text { Late }\end{array}$ & $\begin{array}{c}\text { SWA } \\
\text { Late }\end{array}$ \\
\hline & & & & & & \\
\hline 1 & 8.090 & 8.727 & 8.179 & 9.096 & 8.033 & 9.382 \\
2 & 9.387 & 7.954 & 9.557 & 8.100 & 9.230 & 8.358 \\
3 & 12.381 & 8.759 & 11.139 & 8.604 & 10.880 & 8.855 \\
4 & 9.621 & 8.704 & 9.144 & 8.803 & 9.969 & 8.929 \\
5 & 10.837 & 9.388 & 10.291 & 9.434 & 11.138 & 9.797 \\
6 & 8.775 & 8.286 & 8.527 & 8.435 & 7.366 & 8.577 \\
7 & 8.157 & 7.910 & 8.261 & 8.272 & 8.471 & 8.374 \\
8 & 8.474 & 8.203 & 8.444 & 8.678 & 9.140 & 8.990 \\
9 & 9.701 & 8.976 & 9.967 & 9.524 & 9.467 & 9.723 \\
10 & 11.485 & 8.670 & 11.588 & 9.310 & 12.096 & 9.473 \\
11 & 9.330 & 7.976 & 8.567 & 8.329 & 8.329 & 8.584 \\
15 & 9.593 & 8.862 & 10.385 & 9.356 & 10.249 & 9.216 \\
16 & 6.944 & 9.517 & 6.224 & 9.548 & 5.462 & 10.018 \\
Mean & $\mathbf{9 . 4 4 4}$ & $\mathbf{8 . 6 1 0}$ & $\mathbf{9 . 2 5 2}$ & $\mathbf{8 . 8 8 4}$ & $\mathbf{9 . 2 1 8}$ & $\mathbf{9 . 0 9 8}$ \\
Std Dev & 1.472 & 0.522 & 1.442 & 0.519 & 1.749 & 0.553 \\
\hline
\end{tabular}

Values are means of 3 (mid) and 5 (early and late) min measurement periods.

*Placebo was olive oil treatment used for a companion study. 
Table 11. Cycle Ergometer Total 40 min Energy Expenditure Measurements (kcal) for Indirect Calorimetry and SWA: Placebo* Trial.

\begin{tabular}{|ccc|}
\hline Subject \# & IC & SWA \\
\hline 1 & 324.03 & 355.81 \\
10 & 468.92 & 365.34 \\
11 & 349.69 & 337.32 \\
15 & 403.03 & 365.40 \\
16 & 248.40 & 384.59 \\
2 & 375.66 & 323.46 \\
3 & 458.66 & 349.29 \\
4 & 383.12 & 348.57 \\
5 & 430.20 & 381.11 \\
6 & 328.90 & 336.53 \\
7 & 331.85 & 324.64 \\
8 & 347.43 & 340.56 \\
9 & 388.46 & 374.57 \\
Mean & $\mathbf{3 7 2 . 1 8}$ & $\mathbf{3 5 2 . 8 6}$ \\
Std Dev & 60.42 & 20.31 \\
\hline
\end{tabular}

*Placebo was olive oil treatment used for a companion study. 
Table 12. Cycle Ergometer Exercise Recovery min 4-8 Energy Expenditure (kcal) for Indirect Calorimetry and SWA: Both $\mathrm{CLA}^{*}$ and Placebo** Trials.

\begin{tabular}{|ccccc|}
\hline $\begin{array}{c}\text { Subject } \\
\#\end{array}$ & $\begin{array}{c}\text { IC } \\
\text { CLA }\end{array}$ & $\begin{array}{c}\text { SWA } \\
\text { CLA }\end{array}$ & $\begin{array}{c}\text { IC } \\
\text { Placebo }\end{array}$ & $\begin{array}{c}\text { SWA } \\
\text { Placebo }\end{array}$ \\
\hline & & & & \\
1 & 12.777 & 13.039 & 10.606 & 9.610 \\
2 & 5.911 & 6.755 & 8.318 & 7.310 \\
3 & 9.165 & 8.749 & 8.865 & 8.953 \\
4 & 7.859 & 7.732 & 9.804 & 7.906 \\
5 & & & 12.580 & 10.926 \\
6 & 8.300 & 8.822 & 8.751 & 8.908 \\
7 & 9.578 & 6.479 & 10.026 & 6.615 \\
8 & 7.303 & 7.551 & 8.272 & 8.496 \\
9 & 10.392 & 7.311 & 11.299 & 11.025 \\
10 & 14.055 & 11.869 & 13.229 & 9.674 \\
11 & 9.672 & 8.669 & 7.105 & 6.912 \\
15 & 7.607 & 7.981 & 9.153 & 9.944 \\
16 & 5.490 & 5.923 & 7.603 & 9.359 \\
Mean & $\mathbf{9 . 0 0 9}$ & $\mathbf{8 . 4 0 7}$ & $\mathbf{9 . 6 6 2}$ & $\mathbf{8 . 8 9 5}$ \\
Std Dev & 2.541 & 2.110 & 1.853 & 1.411 \\
\hline
\end{tabular}

${ }^{*} \mathrm{CLA}$ (conjugated linoleic acid) treatment used for a companion study. ${ }^{* *}$ Placebo was olive oil treatment used for a companion study. 
Table 13. Treadmill Exercise Indirect Calorimetry and SWA Energy Expenditure Measurements ( $\mathrm{kcal} / \mathrm{min}$ ).

\begin{tabular}{|c|c|c|c|c|c|c|}
\hline $\begin{array}{c}\text { Subject } \\
\#\end{array}$ & $\begin{array}{c}\text { IC } \\
3 \mathrm{mph}\end{array}$ & $\begin{array}{l}\text { SWA } \\
3 \mathrm{mph}\end{array}$ & $\begin{array}{c}\text { IC } \\
4 \mathrm{mph}\end{array}$ & $\begin{array}{l}\text { SWA } \\
4 \mathrm{mph}\end{array}$ & $\begin{array}{c}\text { IC } \\
4 \mathrm{mph}, 5 \%\end{array}$ & $\begin{array}{c}\text { SWA } \\
4 \mathrm{mph}, 5 \%\end{array}$ \\
\hline 101 & 3.469 & 5.651 & 5.555 & 7.390 & 7.240 & 7.406 \\
\hline 102 & 4.057 & 5.747 & 6.537 & 7.262 & 10.710 & 7.220 \\
\hline 103 & 4.454 & 6.805 & 6.598 & 8.034 & 9.422 & 8.552 \\
\hline 104 & 3.712 & 5.513 & 5.569 & 7.223 & 7.463 & 7.627 \\
\hline 105 & 5.063 & 6.412 & 7.549 & 7.407 & 10.617 & 7.655 \\
\hline 106 & 4.388 & 6.223 & 6.575 & 7.502 & 9.180 & 7.888 \\
\hline 107 & 3.450 & 3.976 & 4.874 & 5.943 & 6.735 & 6.047 \\
\hline 108 & 3.010 & 4.568 & 5.161 & 6.113 & 7.973 & 6.087 \\
\hline 109 & 2.838 & 5.112 & 4.604 & 6.632 & 6.421 & 6.261 \\
\hline 110 & 2.239 & 3.437 & 3.421 & 4.810 & 5.069 & 4.443 \\
\hline 111 & 2.581 & 4.440 & 3.908 & 6.691 & 5.393 & 6.655 \\
\hline 112 & 4.504 & 4.842 & 6.758 & 6.007 & 9.329 & 6.344 \\
\hline 113 & 4.186 & 5.340 & 5.818 & 6.941 & 8.684 & 6.751 \\
\hline 114 & 3.400 & 5.193 & 4.954 & 6.686 & 7.160 & 6.528 \\
\hline 115 & 2.701 & 5.691 & 3.892 & 7.439 & 5.530 & 6.819 \\
\hline 116 & 3.356 & 3.276 & 5.256 & 4.750 & 7.381 & 4.339 \\
\hline 117 & 4.698 & 4.698 & 7.299 & 6.153 & 9.804 & 5.908 \\
\hline 118 & 7.302 & 8.078 & 10.841 & 8.590 & 16.327 & 8.123 \\
\hline 119 & 3.899 & 7.062 & 5.792 & 7.333 & 7.930 & 3.654 \\
\hline 120 & 4.393 & 5.239 & 7.494 & 6.734 & 10.195 & 7.322 \\
\hline Mean & 3.885 & 5.365 & 5.923 & 6.782 & 8.428 & 6.581 \\
\hline Std Dev & 1.115 & 1.188 & 1.665 & 0.960 & 2.525 & 1.283 \\
\hline
\end{tabular}

Values are means of 5 min measurement periods. 
Table 14. Treadmill Exercise Recovery Energy

Expenditure Measurements (kcal) of min 6-10 for Indirect Calorimetry and SWA.

\begin{tabular}{|ccc|}
\hline Subject \# & IC & SWA \\
\hline 101 & 3.805 & 7.2896 \\
102 & 6.501 & 7.0098 \\
103 & 7.422 & 7.3768 \\
104 & 6.88 & 7.8178 \\
105 & 7.23 & 6.8968 \\
106 & 7.494 & 8.0354 \\
107 & 6.012 & 5.0715 \\
108 & 4.031 & 5.7505 \\
109 & 5.457 & 6.1297 \\
110 & 2.571 & 4.7945 \\
111 & 3.765 & 4.5921 \\
112 & 6.677 & 5.8568 \\
113 & 7.541 & 8.0567 \\
114 & 5.59 & 5.9904 \\
115 & 3.709 & 5.2098 \\
116 & 6.381 & 5.6179 \\
117 & 8.768 & 8.5235 \\
118 & 18.239 & 12.4774 \\
119 & 6.44 & 6.4769 \\
120 & 6.817 & 5.7198 \\
Mean & $\mathbf{6 . 5 6 7}$ & 6.735 \\
Std Dev & 3.186 & 1.778 \\
\hline
\end{tabular}




\section{Appendix C}

Statistical Procedures and Results 
Analysis of energy expenditure was performed with two-factor repeated measures ANOVA to test for effects of time, device, and time by device interaction in the ergometer and rest protocols and intensity, device, and their interaction in the treadmill protocol. Paired t-tests were computed to examine differences between average energy expenditure in the various contexts and total energy cost of the ergometer bout as measured by indirect calorimetry and SWA. Pearson's Product Moment correlation analyses were performed to determine associations between the SWA estimate and indirect calorimetry measurement of energy expenditure for each of the measures assessed with the t-test. The reliability of repeated measures of resting energy expenditure on different days by the SWA was examined with Pearson's correlation. Statistical significance was defined at $p<0.05$. Data are presented as means $\pm \mathrm{SD}$. Bland-Altman bias plots were created to assess the agreement between the indirect calorimetry measurement and SWA estimation of energy expenditure as well as the reliability of the SWA estimate-limits of agreement involve the mean difference between the two measurement tools \pm 2 SD of the difference. ${ }^{6}$ Statistical analyses were completed using Statistical Packages for the Social Sciences software (SPSS, Version 11.0 for Windows, 2001, SPSS Inc., Chicago, IL).

Table 15. RM ANOVA for Resting EE Estimate by SWA.

\begin{tabular}{|l|l|l|l|l|l|}
\hline Source & $\mathbf{d f}$ & SS & MS & F & p \\
\hline Trial (2) & 1 & 0.008 & 0.008 & 1.923 & 0.191 \\
\hline Time (4) & 1.638 & 0.002 & 0.001 & 1.679 & 0.214 \\
\hline Trial x Time & 1.907 & 0.0004 & 0.0008 & 0.533 & 0.586 \\
\hline
\end{tabular}

Note: The usual degrees of freedom were corrected by the Huynh-Feldt epsilon value. 
Table 16. RM ANOVA for resting EE Measurement by IC.

\begin{tabular}{|l|c|c|c|c|c|}
\hline Source & df & SS & MS & F & p \\
\hline Trial (2) & 1 & 0.066 & 0.066 & 1.397 & 0.260 \\
\hline Time (4) & 2.829 & 0.049 & 0.017 & 1.682 & 0.191 \\
\hline Trial x Time & 2.554 & 0.005 & 0.002 & 0.211 & 0.859 \\
\hline
\end{tabular}

Note: The usual degrees of freedom were corrected by the Huynh-Feldt epsilon value.

Table 17. Paired T-Test for Pooled Resting EE.

\begin{tabular}{|l|l|l|l|}
\hline & df & $\mathbf{t}$ & $\mathbf{P}$ \\
\hline IC avg - SWA avg & 12 & -0.456 & 0.656 \\
\hline
\end{tabular}


Table 18. Pearson's Correlation for Pooled Resting EE.

\begin{tabular}{|l|l|l|l|}
\hline & $\mathbf{N}$ & $\mathbf{r}$ & $\mathbf{p}$ \\
\hline IC avg - SWA avg & 13 & 0.758 & 0.003 \\
\hline
\end{tabular}

Table 19. Pearson's Correlation for SWA Resting EE—CLA Trial vs. Placebo Trial.

\begin{tabular}{|l|l|l|l|}
\hline & $\mathbf{N}$ & $\mathbf{r}$ & $\mathbf{p}$ \\
\hline Measurement 1 & 13 & 0.933 & 0.000 \\
\hline Measurement 2 & 13 & 0.866 & 0.000 \\
\hline Measurement 3 & 13 & 0.935 & 0.000 \\
\hline Measurement 4 & 13 & 0.900 & 0.000 \\
\hline Average & 13 & 0.932 & 0.000 \\
\hline
\end{tabular}


Table 20. Pearson's Correlation for IC Resting EE-CLA Trial vs. Placebo Trial.

\begin{tabular}{|l|l|l|l|}
\hline & $\mathbf{N}$ & $\mathbf{r}$ & $\mathbf{p}$ \\
\hline Measurement 1 & 13 & 0.553 & 0.050 \\
\hline Measurement 2 & 13 & 0.404 & 0.170 \\
\hline Measurement 3 & 13 & 0.404 & 0.172 \\
\hline Measurement 4 & 13 & 0.542 & 0.056 \\
\hline Average & 13 & 0.569 & 0.043 \\
\hline
\end{tabular}


Table 21. RM ANOVA for Ergometer Exercise EE.

\begin{tabular}{|l|l|l|l|l|l|}
\hline Source & df & SS & MS & F & p \\
\hline Treatment (2) & 1 & 0.105 & 0.105 & 0.097 & 0.762 \\
\hline Time (3) & 1.645 & 0.849 & 0.517 & 1.969 & 0.175 \\
\hline Device (2) & 1 & 13.307 & 13.307 & 2.306 & 0.160 \\
\hline Treatment x Time & 1.870 & 0.052 & 0.028 & 0.312 & 0.721 \\
\hline Treatment x Device & 1 & 0.367 & 0.367 & 0.444 & 0.520 \\
\hline Time x Device & 2 & 2.006 & 1.003 & 13.205 & 0.000 \\
\hline Treatment x Time x Device & 1.953 & 0.050 & 0.026 & 0.388 & 0.679 \\
\hline
\end{tabular}

Note: The usual degrees of freedom were corrected by the Huynh-Feldt epsilon value.

Table 22. RM ANOVA for Ergometer Exercise EE—Placebo Only.

\begin{tabular}{|l|l|l|l|l|l|}
\hline Source & df & SS & MS & F & p \\
\hline Time (3) & 1.852 & 0.233 & 0.126 & 0.760 & 0.470 \\
\hline Device (2) & 1 & 3.783 & 3.783 & 1.027 & 0.331 \\
\hline Time x Device & 1.502 & 1.710 & 1.138 & 8.737 & 0.004 \\
\hline
\end{tabular}

Note: The usual degrees of freedom were corrected by the Huynh-Feldt epsilon value. 
Table 23. Paired T-Test for SWA Estimate vs. IC Measurement of EE in Ergometer Exercise-Placebo Only.

\begin{tabular}{|l|l|l|l|}
\hline Time Period & df & t & p \\
\hline Early & 12 & 1.994 & 0.069 \\
\hline Mid & 12 & 0.900 & 0.386 \\
\hline Late & 12 & 0.237 & 0.817 \\
\hline
\end{tabular}

Table 24. Pearson's Correlation for SWA Estimate vs. IC Measurement of EE in Ergometer Exercise-Placebo Only.

\begin{tabular}{|l|l|l|l|}
\hline Time Period & N & r & p \\
\hline Early & 13 & 0.107 & 0.727 \\
\hline Mid & 13 & 0.116 & 0.707 \\
\hline Late & 13 & 0.029 & 0.924 \\
\hline
\end{tabular}


Table 25. Paired T-Test for SWA Estimate vs. IC Measurement of Relative EE in the Early Time Period Ergometer Exercise-Placebo Only.

\begin{tabular}{|l|l|l|l|}
\hline Time Period & df & $\mathbf{t}$ & $\mathbf{p}$ \\
\hline Early & 12 & 1.573 & 0.142 \\
\hline
\end{tabular}

Table 26. Pearson's Correlation for SWA Estimate vs. IC Measurement of Relative EE in the Early Time Period Ergometer Exercise—Placebo Only.

\begin{tabular}{|l|l|l|l|}
\hline Time Period & N & r & p \\
\hline Early & 13 & 0.301 & 0.318 \\
\hline
\end{tabular}

Table 27. Paired T-Test for Total Ergometer Exercise EE—Placebo Only.

\begin{tabular}{|l|l|l|l|}
\hline & df & $\mathbf{t}$ & $\mathbf{p}$ \\
\hline $\mathbf{I C}_{\text {total }}-\mathbf{S W A}_{\text {total }}$ & 12 & 1.129 & 0.281 \\
\hline
\end{tabular}


Table 28. Pearson's Correlation for Total Ergometer Exercise EE_-Placebo Only.

\begin{tabular}{|l|l|l|l|}
\hline & $\mathbf{N}$ & $\mathbf{r}$ & $\mathbf{p}$ \\
\hline $\mathbf{I C}_{\text {total }}-\mathbf{S W A}_{\text {total }}$ & 13 & 0.106 & 0.731 \\
\hline
\end{tabular}

Table 29. RM ANOVA for Ergometer Exercise EE of Recovery min 4-8.

\begin{tabular}{|l|l|l|l|l|l|}
\hline Source & df & SS & MS & F & p \\
\hline Treatment (2) & 1 & 0.064 & 0.064 & 0.521 & 0.486 \\
\hline Device (2) & 1 & 0.201 & 0.201 & 2.788 & 0.123 \\
\hline Treatment x Device & 1 & 0.099 & 0.099 & 0.052 & 0.823 \\
\hline
\end{tabular}

Table 30. Paired T-Test for Ergometer Exercise EE of Recovery min 4-8-Placebo Only.

\begin{tabular}{|l|l|l|l|}
\hline & df & $\mathbf{t}$ & $\mathbf{p}$ \\
\hline $\mathbf{I C}_{\text {rec }}-\mathbf{S W A}_{\text {rec }}$ & 12 & 1.783 & 0.100 \\
\hline
\end{tabular}


Table 31. Pearson's Correlation for Ergometer Exercise EE of Recovery min 4-8—Placebo Only.

\begin{tabular}{|l|l|l|l|}
\hline & $\mathbf{N}$ & $\mathbf{r}$ & $\mathbf{p}$ \\
\hline $\mathbf{I C}_{\text {rec }}-\mathbf{S W A}_{\text {rec }}$ & 13 & 0.577 & 0.039 \\
\hline
\end{tabular}

Table 32. RM ANOVA for Treadmill Exercise EE.

\begin{tabular}{|l|l|l|l|l|l|}
\hline Source & df & SS & MS & F & p \\
\hline Intensity (3) & 1.069 & 167.593 & 156.767 & 188.656 & 0.000 \\
\hline Device (2) & 1 & 0.767 & 0.767 & 0.274 & 0.607 \\
\hline Intensity x Device & 1.128 & 62.304 & 55.234 & 57.596 & 0.000 \\
\hline
\end{tabular}

Note: The usual degrees of freedom were corrected by the Huynh-Feldt epsilon value. 
Table 33. Paired T-Test for SWA Estimate vs. IC Measurement of EE in Treadmill Exercise.

\begin{tabular}{|l|l|l|l|}
\hline Intensity & df & t & p \\
\hline $\mathbf{3}$ mph, 0\% grade & 19 & -7.270 & 0.000 \\
\hline $\mathbf{4} \mathbf{~ m p h , ~ 0 \% ~ g r a d e ~}$ & 19 & -2.720 & 0.014 \\
\hline $\mathbf{4} \mathbf{~ m p h , ~ 5 \% ~ g r a d e ~}$ & 19 & 3.708 & 0.001 \\
\hline
\end{tabular}

Table 34. Pearson's Correlation for SWA Estimate vs. IC Measurement of EE in Treadmill Exercise.

\begin{tabular}{|l|l|l|l|}
\hline Intensity & $\mathbf{N}$ & $\mathbf{r}$ & $\mathbf{p}$ \\
\hline $\mathbf{3}$ mph, 0\% grade & 20 & 0.693 & 0.001 \\
\hline $\mathbf{4} \mathbf{~ m p h , ~ 0 \% ~ g r a d e ~}$ & 20 & 0.538 & 0.014 \\
\hline $\mathbf{4} \mathbf{~ m p h , ~ 5 \% ~ g r a d e ~}$ & 20 & 0.472 & 0.035 \\
\hline
\end{tabular}


Table 35. Paired T-Test for SWA Estimate vs. IC Measurement of EE in Treadmill Exercise-Males Only.

\begin{tabular}{|l|l|l|l|}
\hline Intensity & df & t & p \\
\hline 3 mph, 0\% grade & 9 & -8.598 & 0.000 \\
\hline 4 mph, 0\% grade & 9 & -2.182 & 0.057 \\
\hline 4 mph, 5\% grade & 9 & 2.888 & 0.018 \\
\hline
\end{tabular}

Table 36. Pearson's Correlation Coefficient for SWA Estimate vs. IC Measurement of EE in Treadmill Exercise-Males Only.

\begin{tabular}{|l|l|l|l|}
\hline Intensity & $\mathbf{N}$ & $\mathbf{r}$ & $\mathbf{p}$ \\
\hline 3 mph, 0\% grade & 10 & 0.81 & 0.004 \\
\hline 4 mph, 0\% grade & 10 & 0.85 & 0.002 \\
\hline 4 mph, 5\% grade & 10 & 0.38 & 0.283 \\
\hline
\end{tabular}


Table 37. Paired T-Test for SWA Estimate vs. IC Measurement of EE in Treadmill Exercise-Females Only.

\begin{tabular}{|l|l|l|l|}
\hline Intensity & df & t & p \\
\hline 3 mph, 0\% grade & 9 & -3.581 & 0.006 \\
\hline 4 mph, 0\% grade & 9 & -1.676 & 0.128 \\
\hline 4 mph, 5\% grade & 9 & 2.326 & 0.045 \\
\hline
\end{tabular}

Table 38. Pearson's Correlation Coefficient for SWA Estimate vs. IC Measurement of EE in Treadmill Exercise-Females Only.

\begin{tabular}{|l|l|l|l|}
\hline Intensity & $\mathbf{N}$ & $\mathbf{r}$ & $\mathbf{p}$ \\
\hline 3 mph, 0\% grade & 10 & 0.24 & 0.498 \\
\hline 4 mph, 0\% grade & 10 & 0.08 & 0.833 \\
\hline 4 mph, 5\% grade & 10 & 0.29 & 0.415 \\
\hline
\end{tabular}


Table 39. Paired T-Test for Treadmill Exercise EE of Recovery min 6-10.

\begin{tabular}{|l|l|l|l|}
\hline & df & t & $\mathbf{p}$ \\
\hline IC $_{\text {rec }}-$ SWA $_{\text {rec }}$ & 19 & -0.418 & 0.680 \\
\hline
\end{tabular}

Table 40. Pearson's Correlation for Treadmill Exercise EE of Recovery min 6-10.

\begin{tabular}{|l|l|l|l|}
\hline & $\mathbf{N}$ & $\mathbf{r}$ & $\mathbf{p}$ \\
\hline $\mathbf{I C}_{\text {rec }}-\mathbf{S W A}_{\text {rec }}$ & 20 & 0.889 & 0.000 \\
\hline
\end{tabular}




\section{Power Analysis}

Assuming: 2 independent groups $(\mathrm{a}=$ number of groups $=2), \mathrm{n}$ observations/group

Estimates: $\quad \hat{\sigma}^{2}=\mathrm{MS}_{\mathrm{Within}}=3.683$

$$
\text { Groups means: } \quad \begin{aligned}
\overline{\mathrm{Y}}_{1} & =9.444 \text { for IC } \\
& \overline{\mathrm{Y}}_{2}=8.610 \text { for SWA }
\end{aligned}
$$

Overall average: $\quad \overline{\bar{Y}}=9.027$

How groups differ from overall average: $\sum \alpha_{i}^{2}=\sum\left(\bar{Y}_{i}-\overline{\bar{Y}}\right)^{2}=0.348$

Non-centrality parameter: $\lambda$

$$
\lambda=\mathrm{n} \sum \alpha_{\mathrm{i}}^{2} / \hat{\sigma}^{2}=\mathrm{n}(0.348) / 3.683=\mathrm{n}(0.094)
$$

Function of non-centrality parameter, used on the chart: $\phi$

$$
\begin{gathered}
\phi=\sqrt{\lambda / \mathrm{a}}=\sqrt{n(.094) / 2}=\sqrt{\mathrm{n}}(0.217) \\
\mathrm{df}_{\text {numerator }}=\mathrm{a}-1=1 \quad \mathrm{df}_{\text {deno min ator }}=\mathrm{a}(\mathrm{n}-1)=2(\mathrm{n}-1)
\end{gathered}
$$

Table 41. Finding the number of observations (n) needed to have a power equal to 0.80 .

\begin{tabular}{|l|l|l|l|l|}
\hline $\mathbf{n}$ & $\mathrm{df}_{\text {denomin ator }}$ & $\phi$ & Power & Conclusion \\
\hline 20 & 38 & 0.972 & $\sim 0.3$ & Increase $\mathrm{n}$ \\
\hline 50 & 98 & 1.536 & 0.55 & Increase $\mathrm{n}$ \\
\hline 100 & $198 \approx \infty$ & 2.17 & 0.86 & Decrease $\mathrm{n}$ \\
\hline 80 & 158 & 1.941 & 0.80 & Keep $\mathrm{n}$ \\
\hline
\end{tabular}

Power determined using Pearson-Hartley Power Charts. ${ }^{56}$ 
Appendix D

Institutional Review Board Proposal 


\section{Request for Approval of Research Proposal \\ Department of Human Nutrition, Foods, and Exercise Virginia Tech}

TITLE: Effects of trans-10, cis-12 Conjugated Linoleic Acid (CLA) on Metabolic Rate and Fuel Use at Rest and During Exercise

INVESTIGATORS: $\quad$ Janet Walberg Rankin, Ph.D.

Professor

Department of Human Nutrition, Foods, and Exercise

Max Shute, MS.

Doctoral Graduate student

Department of Human Nutrition, Foods, and Exercise

\section{Justification of Project}

Many individuals would like to reduce their body fat and look for dietary supplements to help achieve this goal. Recently, conjugated linoleic acid (CLA) has been demonstrated to dramatically decrease fat mass in animal models. The hypothesis that CLA may potentiate the fat-utilizing effect of aerobic exercise and minimize the loss of lean tissue in dieting humans has not been tested.

Major food sources of CLA include dietary lipids originating from ruminant animals (e.g. milk, beef). CLA consumption by adults in the United States is estimated at $1 \mathrm{~g} /$ person/d (Zambell et al, 2000). Currently, numerous studies are evaluating other health effects of CLA including increased immune function and anticarcinogenic properties.

Body fat reduction has been observed with CLA supplementation in a number of animal studies (Azain et al, 2000, Delany et al, 1999). For example, Park et al (1997) observed a 57 and $60 \%$ reduction of body fat, in male and female mice respectively, with a CLA supplemented diet (0.5\%) along with a 5\% and $14 \%$ increase in lean body mass. Other studies have demonstrated rapid body fat reduction within 2 wks.

There has been some study of the effectiveness of CLA on health, body composition, and metabolic rate in humans. Some studies find no effect (Vessby et al. 1999, Zambell et al. 2000) while others report modest reductions in body fat or weight (Blankson et al., 2000, Riserus et al. 2001). For example, Blankson et al (2000), administered CLA in doses of 1.7, $3.4,5.1$, and $6.8 \mathrm{~g} /$ day for 12 weeks to 60 overweight or obese volunteers. A similar, significant reduction in body fat mass was observed in the 3.4 and $6.8 \mathrm{~g}$ CLA groups, suggesting that doses between 3 and $4 \mathrm{~g} / \mathrm{d}$ are effective.

Aerobic exercise is typically recommended as part of a weight reduction effort since it has been shown to increase metabolic rate and fat utilization during and in the short-term period after exercise. Measurement of respiratory exchange ratio (RER) involves assessment of expired gas volume and content of oxygen and carbon dioxide, during exercise is used as a tool to determine whether specific substances influence fat use. Acute aerobic exercise increases metabolic rate and causes a shift towards fat oxidation during and post exercise. Because it has been suggested that CLA enhances fat oxidation, it is possible that CLA supplementation would further enhance fat use with exercise. It is possible that CLA may 
potentiate the effects of aerobic exercise leading to a more efficient means of fat loss in dieting humans.

\section{Statement of the Problem}

In summary, CLA supplementation has been shown to result in dramatically decreased body fat in rodents and modestly decreased body fat in humans. Some recent research suggests a particular isomer of CLA, trans-10, cis-12, is the form with most effect on body fat in rodents (Park et al. 1999). Thus, DeLany and West (2000) suggest that "most of the studies conducted so far must be repeated using the purified isomers". A recent test of the acute effect of a mixture of CLA isomers showed that a single dose increased metabolic rate and fat utilization in mice over $2 \mathrm{~h}$ after ingestion (Ohnuki et al. 2001). Some studies in humans find a modest reduction in body fat and fat (Blankson et al. 2000, Riserus et al. 2001) while some find no effect (Vessby et al, Zambell et al. 2000). It is noteworthy that none of the published tests of CLA in humans has used the purified trans-10, cis-12 isomer. In addition, none has tested the potential synergistic effect of CLA with exercise, another stimulus for an increase in metabolic rate and fat use. The effect of CLA on humans has not been conclusive although it is currently being marketed as an aid for fat loss and muscle building. This study will examine the dose response effects of acute consumption of trans-10,cis-12 isomer of CLA on metabolic rate and fuel use at rest and during submaximal exercise.

Thus, the purpose of this study is to examine the effects of oral trans-10, cis-12 CLA on resting and exercise metabolic rate and fuel use.

\section{Procedures}

\section{Subject selection}

Fifteen 18-25 year-old normal weight, untrained males will be recruited for participation as subjects. We anticipate recruiting subjects via posted fliers and e-mail announcements. Those who respond will be told of the general plan for the study. Those still interested will be invited to a group or an individual session to hear the details of participation and potential risks. They will be given a chance to ask any questions. Those still interested will receive a copy of the informed consent. Those who return this document will be considered during subject selection. Subjects will undergo a health screening and will be excluded if conditions such as diabetes, orthopedic limitations or injuries, epilepsy or seizures, hypoglycemia, mental illness, pulmonary disease, history of eating disorders, or other restrictions exist.

\section{General Design}

Baseline measurements will be performed on all subject: body fat and aerobic fitness (see below). Following the baseline testing, each subject will come in six additional times to our laboratory for approximately 2 hours each time in the morning before having eaten anything. There will be at least 2 days between each test. Three of these tests will be resting tests (resting the entire time) while three will include a submaximal exercise test. Subjects will ingest capsules for each trial. Sometimes these will be CLA (2.4 or $4.8 \mathrm{~g})$ and sometimes these will be a placebo (olive oil). For each of these trials, we will take 4 blood 
samples over 2 hours and take multiple measures of metabolic rate and use of carbohydrate or fat.

Aerobic fitness $\left(\mathrm{VO}_{2}\right.$ peak) will be determined for all subjects using a graded exercise test on a cycle ergometer (Monark 818E Ergomed C). Prior to $\mathrm{VO}_{2}$ peak testing subjects will be familiarized with bicycle exercise at a constant pedaling rate and while breathing through the mouthpiece and breathing valve. Test will begin at $75 \mathrm{w}$ while pedaling at $70 \mathrm{rpm}$ and will increase $25 \mathrm{w}$ every 2 min until the subject can no longer maintain $70 \mathrm{rpm}$. Continuous indirect calorimetry will be performed using the CPX metabolic system (MedGraphics Corporation, St. Paul, MN). The subjects will breath into a mouthpiece during the entire exercise test. Excluding warm up and cool down, the test will likely last between 10 and 15 minutes.

Body fat percentage will be determined by skinfold measurement at three sites: thigh, chest, abdomen. This involves using a caliper to measure the thickness of a fold of skin picked up at the listed sites.

Resting Metabolic Rate and Fuel Use: Each subject will come to our laboratory six times, in the morning, without eating breakfast, taking any medication or supplement, using any tobacco products, doing any exercise. We will measure resting metabolic rate $(\mathrm{kcal} / \mathrm{min})$. This requires lying down for approximately 15 minutes followed by collection of expired air every 20 minutes for two hours. Each collection period will last 10 minutes. This involves breathing normally while in a metabolic hood that collects and analyzes expired air. This measurement can determine metabolic rate $(\mathrm{kcal} / \mathrm{min})$ as well as the amount of carbohydrate and fat being used.

Blood collection: A blood sample will be taken after the first measurement of resting metabolic rate and just before ingestion of the supplement. Additional samples will be collected 60, 90 and 120 minutes after supplement ingestion (same timing for rest and exercise trials). The amount in each sample is about two teaspoons. All blood sampling will be performed by a Certified Medical Laboratory Technician experienced in the procedure. Your blood sample will be tested for HIV if an experimenter becomes exposed to your blood during collection or later during analysis

Submaximal exercise test: On exercise trial days, 45 minutes after ingestion of the capsules, the subject will pedal on a stationary cycle for 40 minutes at a moderate intensity (about $60 \%$ of their pre-determined maximal fitness level). As described above, blood samples and metabolic rate measurements will be conducted several times during the exercise test.

Diet: We will ask each subject to record their diet for a full day prior to the first of the six trials. They will then be asked to consume the same diet on the day prior to each of the next five trials. This is important since food consumption can affect the ability to estimate metabolic rate and fuel use. 


\section{Risks and Benefits}

$\mathrm{VO}_{2}$ peak testing increases the short-term risk of cardiovascular events such as sudden death and myocardial infarction. However, the subjects we intend to use are at very low risk because of their young age, high activity level, and screening to eliminate those with elevated risk. Subjects selected will be in the "low risk" stratification according to ACSM guidelines(2000), i.e. women less than 50 years of age, asymptomatic of disease, and are positive for no more than one of the following risk factors: family history, cigarette smoking, hypertension, hypercholesterolemia, impaired fasting glucose, obesity, and sedentary lifestyle. Fatigue, muscle soreness, and muscle strains could result from the exercise tests but the latter is unlikely due to the high activity level of the subjects and the safety of stationary biking.

The safety of human consumption of CLA has been researched over 12 weeks at doses up to $6.8 \mathrm{~g} / \mathrm{d}$ with no clinically adverse effects observed (Blankson et al 2000). Subjects were evaluated for blood lipids (TG, LDL and HDL cholesterol), hematological factors (erythrocytes, WBC, platelets), blood electrolytes $\left(\mathrm{Ca}^{+}, \mathrm{Cl}^{-}, \mathrm{K}^{+}, \mathrm{Na}^{+}\right)$, and vital signs. Other research, reported only as an abstract, has utilized doses as high as $7.2 \mathrm{~g}$ /day over 6 weeks with no reported side effects in humans (Lowery et al, 1997). Research by Vessby et al. (1999) and Blankson et al. (2000)have reported adverse gastrointestinal symptoms in both CLA and placebo (oil) groups. We will instruct subjects to consume capsules directly with a meal and water to decrease gastro-intestinal problems.

Risks of the blood withdrawal include bruising and very limited risk of infection. All blood draws will be taken using sterile equipment by a Certified Medical Laboratory Technician experienced in the procedure. Universal precautions will be taken in collection of all blood samples.

Subjects will be financially compensated $\$ 5$ for completion of baseline testing and an additional $\$ 70$ for the full completion of the study. Additionally, they will receive information on their body composition and aerobic capacity.

\section{Confidentiality}

The data from this study will be kept strictly confidential. No data will be released to anyone but the principal investigator and graduate students involved in the project without written consent of the subject. Data will be identified by subject number.

\section{Biographical Sketch}

Janet Walberg Rankin is a Professor in the Department of Human Nutrition, Foods, and Exercise. She has been on the faculty at Virginia Tech since 1982. She earned her B.S. in Zoology from Duke University in 1977 and her Ph.D. in Nutrition from the University of California at Davis in 1982. Her research is primarily in sports nutrition and weight control for obese individuals. Publications of her research has appeared in journals such as International Journal of Sport Nutrition, Medicine and Science in Sports and Exercise, International Journal of Sports Medicine, and Journal of Nutrition Education. 
Max Shute is a graduate student/teaching assistant in the Department of Human Nutrition, Foods, and Exercise. He earned his B.S. in Nutrition from Western Illinois University in 1993 and a Masters in Exercise Science from Appalachian State University in 2000. His research involvement during his Master's program included studies of the immune response to exercise, weight control for the obese, equipment validation. He is currently assisting in metabolic rate assessment of subjects in a research project of Dr. Rankin's regarding postexercise food consumption and muscle metabolism.

\section{REFERENCES}

1. Azain, M.J., Hausman, D.B., Sisk, M.B., Flatt, W.P., Jewell, D.E. (2000). Dietary conjugated linoleic acid reduces rat adipose tissue cell size rather than cell number. $J$. Nutr. 130: 1548-1554.

2. Blankson, H., Stakkestad, J., Fagertun, H., Thom, E., Wadstein, J., Gudmundsen, O. (2000). Conjugated linoleic acid reduces body fat mass in overweight and obese humans. J. Nutr. 130:2943-2948.

3. DeLany, J., Blohm, F., Truett, A., Scimeca, J., West, D. (1999). Conjugated linoleic acid rapidly reduces body fat content in mice without affecting energy intake. Am. J. Physiol. 276:R1172-R1179.

4. DeLany, J., West, D., (2000). Changes in body composition with conjugated linoleic acid. Am. Coll. Nutr. 19:487S-493S.

5. Lowery, L., Appicelli, P., Lemon, P. (1997). Conjugated linoleic acid enhances muscle size and strength gains in novice body builders. Abstract Med. Sci. Sports Exer. S182.

6. Ohnuki, K., Haramizu S., Oki K., Ishihara K., and Fushiki T., (2001) A single oral administration of conjugated linoleic acid enhanced energy metabolism in mice. Lipids 36:583-587.

7. Park, Y., Albright, K., Liu, W., Storkson, J., Cook, M., Pariza, M., (1997). Effect of conjugated linoleic acid on body composition in mice. Lipids. 32:853-858.

8. Park, Y., Storkson, M., Albright, K., Liu, W., Pariza, M., (1999). Evidence that the trans-10, cis-12 isomer of conjugated linoleic acid induces body composition changes in mice. Lipids. 34:235-241.

9. Riserus, U. Bergulund, L., and Vessby, B. (2001) Conjugated linoleic acid (CLA) reduced abdominal adipose tissue in obese middle-aged men with signs of the metabolic syndrome: a randomized controlled trial. Int. J. Obesity 25:1129-1135.

10. Vessby, B., Smedman, A., (1999). Conjugated linoleic acid (CLA) reduces body fat content in humans. Abstract from Chemistry and Physics of Lipids. T2/01:152.

11. West, D., DeLany, P., Camet, P., Blohm, F., Truett, A., Scimeca, J., (1998). Effects of conjugated linoleic acid on body fat and energy metabolism in the mouse. Am. J. Physiol. 275:R667-R672.

12. Zambell, K., Keim, N., Van Loan, M., Gale, B., Benito, P., Kelley, D., Nelson, G., (2000). Conjugated linoleic acid supplementation in humans: effects on body composition and energy expenditure. Lipids. 35:777-782. 


\section{Request for Approval of Research Proposal \\ Department of Human Nutrition, Foods, and Exercise Virginia Tech}

Title: The reliability and validity of the SenseWear Armband in the measurement of energy expenditure

Investigators: Janet Walberg Rankin, Ph.D.

Professor

Department of Human Nutrition, Foods, and Exercise

Margaret Fruin

Master's Graduate student

Department of Human Nutrition, Foods, and Exercise

\section{Justification of Project}

Approximately $55-60 \%$ of American adults are overweight (body mass index $(\mathrm{BMI}) \geq$ $\left.25 \mathrm{~kg} / \mathrm{m}^{2}\right)$ or obese $\left(\mathrm{BMI} \geq 30 \mathrm{~kg} / \mathrm{m}^{2}\right)$ (Jakicic et al, 2001). The obesity rates are particularly alarming because an association has been shown to exist between obesity and heart disease, diabetes, hyperlipidemia, and other health conditions. Multiple factors affect weight gain and obesity including the positive influence of physical activity. Many interventions have been developed in an attempt to increase activity but determination of success has been hampered by relatively poor methods to assess activity and energy expenditure.

The gold standards in the measurement of energy expenditure are indirect calorimetry and doubly labeled water. Indirect calorimetry requires a laboratory setting or a metabolic chamber and is therefore not useful in assessing energy expenditure in free-living conditions. Doubly labeled water (DLW) is a valid technique for determining energy expenditure in freeliving individuals over 3 days to 3 weeks, but the expense of the labeled water and analysis make this method unpractical and inaccessible to many.

Tools have been developed to provide estimates of energy expenditure, and their validity has been compared to indirect calorimetry, DLW or both. Physical activity records and recall questionnaires are commonly used by many researchers, but both measures have rarely been shown to result in accurate estimations of energy expenditure. For example, Irwin et al. (2001) found that men overreported their energy expenditure on physical activity records by $7.9 \pm 3.2 \%$ compared to DLW and overestimated their energy expenditure with a recall questionnaire by an even greater degree $(30.6 \pm 9.9 \%)$.

Pedometers were developed to assess physical activity based on the number of steps a person takes per day. Although these devices may be useful to help individuals monitor their level of daily walking activity, they have been shown to be poor estimators of energy expenditure. Leenders et al. (2001) found that the Yamax-Digiwalker-500 (a pedometer) underestimated the daily physical activity energy expenditure of 13 women by $\sim 59 \%$ when compared to DLW.

Heart rate monitors are often used by those engaging in exercise bouts to monitor their intensity but have been shown to not be accurate in the measurement of daily living energy expenditure. Some studies have found heart rate corresponds linearly with energy 
expenditure at high activity levels, but a weak relationship exists at low levels (Luke et al, 1997; Meijer et al, 1989).

Accelerometers have been designed to detect the accelerations of the body, which are related to the energy expenditure generated to produce that acceleration (Montoye et al, 1996). Uniaxial accelerometers detect acceleration in the vertical plane but have been shown to lack the ability to accurately assess energy expenditure of running (Haymes et al, 1993) and other higher intensity activities and are poor measures of energy expenditure in daily living activities (Hendelman et al, 2000). Triaxial accelerometers detect the accelerations of the body in three planes and have been shown to be better estimators of energy expenditure than the previous tools discussed, but they still have their limitations. Triaxial accelerometers not only underestimate high intensity running energy expenditure (Meijer et al, 1989; Jakicic et al, 1999), but they also cannot detect the increased energy expenditure of walking on an incline (Levine et al., 2001) or recovering from exercise (Sherman et al, 1998). Non-ambulatory physical activity such as cycling (Jakicic et al, 1999) and some activities in daily life (Leenders et al, 2001) also cannot be adequately detected by triaxial accelerometers, which leads to an inaccurate energy expenditure estimation.

The SenseWear Armband (BodyMedia, Inc) is the latest tool for assessing energy expenditure that can be used in free-living conditions. The Armband obtains raw physiologic data from each of its sensors (a 2-axis accelerometer, heart rate monitor, heat flux sensor, skin temperature thermistor, near-body ambient temperature sensor, and galvanic skin response sensor) that is combined and used in proprietary algorithms to estimate energy expenditure in calories. The SenseWear Armband is promoted as providing an accurate estimation of free-living EE, although no studies have been published that determine the reliability or accuracy of this device. Experiments testing the validity of the SenseWear Armband measurement compared to indirect calorimetry are needed before this tool can be used in field experiments.

\section{Statement of the Problem}

The SenseWear Armband is the latest energy expenditure assessment tool for use outside laboratory conditions. If proven to generate reliable and accurate energy expenditure measurements, health professionals, individuals, and researchers will benefit enormously due to its variety of uses. Before the SenseWear Armband can be tested in free-living conditions and eventually utilized publicly, laboratory tests must be performed to determine its validity in rest and exercise bouts compared with indirect calorimetry measurements of energy expenditure.

Thus, the purpose of this study is to determine the accuracy of the SenseWear Armband in measuring energy expenditure at rest and during steady-state walking on a treadmill with and without an inclination.

\section{Procedures \\ Subject selection}

Sixteen healthy men and women, aged 18-35 years old will be recruited. They will be screened for risk factors (see attached health history form) that would normally exclude them from physical activity participation, including conditions such as orthopedic limitations or injuries, epilepsy or seizures, cardiovascular diseases, diabetes, mental illness, pulmonary 
disease, hypoglycemia, history of eating disorder, or other restrictions. The subjects will be thoroughly informed on the details of the study and potential risks.

\section{General design}

Baseline measurements will be performed on all subjects including weight, height, and body fat percentage. Body fat percentage will be determined by taking measurements of the thickness of folds of skin at the abdomen, chest, and thigh for the men and at the waist, thigh, and back of the arm for the women. The skin fold thickness measurements will be used in the body density formulas devised by Jackson and Pollock $(1978,1980)$ to determine an estimate of body fat percentage. The same technician will perform all measurements.

The SenseWear Armband and Polar heart rate monitor will be worn for approximately 30 minutes before any exercise or data collection in order to allow the Armband to get accustomed to the subject. The subject will then stand at rest on the treadmill while their resting energy expenditure is measured via indirect calorimetry for 10 minutes. Following a brief warm-up the subject will perform 30 minutes of moderate, walking exercise. Specifically, they will walk on a treadmill at $3 \mathrm{mph}$ for 10 minutes, $4 \mathrm{mph}$ for 10 minutes, and then $4 \mathrm{mph}$ at an inclination of 5 degrees for another 10 minutes. Following the exercise another 10-minute indirect calorimetry measurement will be made while the subject stands at rest. Continuous indirect calorimetry will be performed using the Sensor Medics metabolic system. Comparisons between the energy expenditure measurements and the SenseWear Armband output for the last 5 minutes of each 10-minute segment will be made.

The indirect calorimetry measurements involve the use of a computer analysis system that connects to the subject by tubes and a mouthpiece. The mouthpiece is rubber and similar to a snorkel mouthpiece. The amount of oxygen consumed, the amount of carbon dioxide expired, and the proportion of fat or carbohydrate fuel used by the subject is determined. The energy expenditure of the subject can then be determined by use of a table and simple equation. The SenseWear Armband is smaller than a walkman (approximately 3.5"x3.0"x.75") and is worn around the upper arm by use of an elastic band. The armband contains an accelerometer to measure motion of the body, a heat flux sensor, galvanic skin response sensor, and skin temperature sensor. All of this raw sensor data is then converted into an estimate of energy expenditure by use of proprietary algorithms.

\section{Risks and Benefits}

The armband (SenseWear Armband, Bodymedia, Inc.) does not pose any risk to the subject. It is similar to wearing a heart rate monitor. The exercise is low intensity walking and low risk, but minimal risks are still possible (e.g. sprained ankle, twisted knee, or minor abrasion if one fell while on the treadmill). No benefits will be promised to subjects participating in this study.

\section{Confidentiality}

The data from this study will be kept strictly confidential. No data will be released to anyone but the principal investigator and graduate students involved in the project without written consent of the subject. Data will be identified by subject number.

\section{Biographical Sketch}

Janet Walberg Rankin is a Professor in the Department of Human Nutrition, Foods, and Exercise. She has been on the faculty at Virginia Tech since 1982. She earned her B.S. in 
Zoology from Duke University in 1977 and her Ph.D. in Nutrition from the University of California at Davis in 1982. Her research is primarily in sports nutrition and weight control for obese individuals. Publications of her research have appeared in journals such as International Journal of Sport Nutrition, Medicine and Science in Sports and Exercise, International Journal of Sports Medicine, and Journal of Nutrition Education.

Margaret Fruin is a graduate student/teaching assistant in the Department of Human Nutrition, Foods, and Exercise. She earned her B.S. degrees in Human Nutrition and in Kinesiology from Kansas State University in 2001. She is currently assisting in a research project of Dr. Rankin's regarding trans-10, cis-12 conjugated linoleic acid on metabolic rate and fuel use at rest and during exercise. This study involves use of the SenseWear Armband. Thus, she will have experience using this equipment.

\section{References}

1. Haymes EM, Byrnes WC. Walking and running energy expenditure estimated by Caltrac and indirect calorimetry. Med Sci Sports Exerc 1993;25:1365-1369.

2. Hendelman D, Miller K, Baggett C, Debold E, Freedson P. Validity of accelerometry for the assessment of moderate intensity physical activity in the field. Med Sci Sports Exerc 2000;32(suppl):S442-S449.

3. Irwin ML, Ainsworth BE, Conway JM. Estimation of energy expenditure from physical activity measures: determinants of accuracy. Obesity Research 2001;9:517-525.

4. Jackson AS, Pollock ML. Generalized equations for predicting body density in men. Br J Nutr 1978;40:497-504.

5. Jackson AS, Pollock ML, Ward A. Generalized equations for predicting body density in women. Med Sci Sports Exerc 1980;12:175-182.

6. Jakicic JM, Clark K, Coleman E, Donnelly JE, Foreyt J, Melanson E, Volek J, Volpe SL. ACSM position stand on the appropriate intervention strategies for weight loss and prevention of weight regain for adults. Med Sci Sports Exerc 2001;33:2145-2156.

7. Jakicic JM, Winters C, Lagally K, Ho J, Robertson RJ, Wing RR. The accuracy of the TriTrac-R3D accelerometer to estimate energy expenditure. Med Sci Sports Exerc 1999;31:747-754.

8. Leenders NJM, Sherman WM, Nagaraja HN, Kien CL. Evaluation of methods to assess physical activity in free-living conditions. Med Sci Sports Exerc 2001;33:1233-1240.

9. Levine JA, Baukol PA, Westerterp KR. Validation of the Tracmor triaxial accelerometer system for walking. Med Sci Sports Exerc 2001;33:1593-1597.

10. Luke A, Maki KC, Barkey N, Cooper R, McGee D. Simultaneous monitoring of heart rate and motion to assess energy expenditure. Med Sci Sports Exerc 1997;29:144-148.

11. Meijer GA, Westerterp KR, Koper H, Ten Hoor F. Assessment of energy expenditure by recording heart rate and body acceleration. Med Sci Sports Exerc 1989;21:343-347.

12. Montoye HJ, Kemper HCG, Saris WHM, Washburn RA. Measuring physical activity and energy expenditure. Champaign, IL: Human Kinetics, 1996.

13. Sherman WM, Morris DM, Kirby TE, Petosa RA, Smith BA, Frid DJ, Leenders N.

Evaluation of a commercial accelerometer (TriTrac-R3D) to measure energy expenditure during ambulation. Int J Sports Med 1998;19:43-47. 
Appendix E

Informed Consent and Health History Forms 


\title{
Informed Consent for Participants of Investigative Projects
}

\author{
Department of Human Nutrition, Foods, and Exercise \\ Virginia Tech
}

TITLE: Effects of Conjugated Linoleic Acid on Metabolic Rate and Fuel Use at Rest and During Exericse

PRINCIPAL INVESTIGATORS: Janet Walberg Rankin, Ph.D., Max Shute, MS.

PURPOSE: Conjugated linoleic acid (CLA) is a fatty acid naturally found in foods such as milk and beef. Recent research has reported that CLA supplementation in animals decreased body fat while increasing lean tissue (Delany et al. 1999, West et al. 1998). Some recent research suggests a particular isomer of CLA, trans-10, cis-12, is the form with most effect on body fat in rodents (Park et al. 1999). Thus, DeLany and West (2000) suggest that "most of the studies conducted so far must be repeated using the purified isomers". A recent test of the acute effect of a mixture of CLA isomers showed that a single dose increased metabolic rate and fat utilization in mice over $2 \mathrm{~h}$ after ingestion (Ohnuki et al. 2001). There has been some study of the effectiveness of CLA on health, body composition, and metabolic rate in humans. Some studies find no effect (Zambell et al. 2000) while others report modest reductions in body fat or weight (Blankson et al., 2000, Riserus et al. 2001). It is noteworthy that none of the published tests of CLA in humans has used the purified trans-10, cis-12 isomer. In addition, none has tested the potential synergistic effect of CLA with exercise, another stimulus for an increase in metabolic rate and fat use. The effect of CLA on humans has not been conclusive although it is currently being marketed as an aid for fat loss and muscle building. This study will examine the dose response effects of acute consumption of trans-10,cis-12 isomer of CLA on metabolic rate and fuel use at rest and during submaximal exercise.

\section{General Design}

We will do some baseline measurements that include your body fat (measure the thickness of fat folds at three areas of your body: triceps, abdomen, and thigh) and your aerobic fitness (see below). Following the baseline testing, you will come in four additional times to our laboratory for approximately 3.5 hours each time in the morning before you have eaten anything. There will be at least 2 days between each test. Two of these tests will be resting tests (you rest the entire time) while two will include a submaximal exercise test. You will ingest capsules for each trial. Sometimes these will be CLA (4.8 g) and sometimes these will be a placebo (olive oil). For each of these trials, we will take 4 blood samples over 3 hours and take multiple measures of your metabolic rate and use of carbohydrate or fat.

Maximum oxygen consumption testing: This test will measure your aerobic fitness. You will cycle at 70rpm on a stationary bike starting at a comfortable resistance ( 75 watts) for 2 minutes. The resistance will increase 25 watts every 2 minutes until exhaustion or you can no longer maintain 70rpm. During this test you will be breathing room air through a mouthpiece similar in nature to a snorkel. 
Resting Metabolic Rate and Fuel Use: You will come to our laboratory, in the morning, without eating breakfast, taking any medication or supplement, smoking any tobacco products, or doing any exercise. We will measure your resting metabolic rate (RMR) which is the amount of calories you burn per day if you were to lay in bed without getting up or sleeping. This requires you to lie down for approximately 15 minutes followed by collect your expired air every 20 minutes for two hours. Each collection period will last 10 minutes. This involves having your breath normally while in a metabolic hood that collects and analyzes your expired air. At this time you will also being wearing an armband monitor similar in size to a walkman that will also be measuring your metabolism. This measurement can determine your metabolic rate $(\mathrm{kcal} / \mathrm{min})$ as well as the amount of carbohydrate and fat you are using.

Blood collection: A blood sample will be taken after the first measurement of resting metabolic rate and just before ingestion of the supplement. Additional samples will be collected 80,110 and 140 minutes after supplement ingestion (same timing for rest and exercise trials). The amount in each sample is about two teaspoons. All blood sampling will be performed by a Certified Medical Laboratory Technician experienced in the procedure. Your blood sample will be tested for HIV if an experimenter becomes exposed to your blood during collection or later during analysis

Submaximal exercise test: On exercise trial days, 80 minutes after you ingest your capsules, you will pedal on a stationary cycle for 40 minutes at a moderate intensity (about $60 \%$ of your maximal fitness level). During the test you will also be wearing the armband. As described above, blood samples and metabolic rate measurements will be conducted several times during the exercise test.

Diet: We will ask you to record your diet for a full day prior to your first of the six trials. You should consume the same diet on the day prior to each of the next five trials. This is important since what you eat can affect the ability to estimate your metabolic rate and fuel use.

SUMMARY OF SUBJECT RESPONSIBILITIES

1. Refrain from taking any other nutritional supplements without checking first with the experimenters.

2. Give maximal effort on performance tests.

3. Come to each of the exercise training tests in a fasted condition (nothing to eat since the evening before) without having consumed any alcoholic or caffeinated beverages the night before or that morning or smoked any cigarettes, pipes, or cigars that morning.

4. Inform the experimenters if you experience any unusual symptoms from any of the testing, or supplements.

5. Inform the researchers of any known medical conditions or allergies you are aware of prior to the study as well as any transmittable diseases acquired during the study.

RISKS OF PARTICIPATION 
1. CLA supplements in doses greater than you will consume over longer time periods have not been shown to cause any clinical changes in blood measures or vital signs in humans. For example, one study had overweight women consume $6.8 \mathrm{~g} / \mathrm{d}$ of CLA over 12 weeks. Blood and vital signs (e.g. blood pressure, heart rate) were measured regularly to determine safety of the supplementation. No clinically adverse effects were observed in the various factors that included blood lipids (triglyceride, low-density lipoprotein, high density lipoprotein), hematological factors (red blood cells, white blood cells, platelets), blood electrolytes (calcium, potassium, chloride). A similar proportion of subjects consuming the CLA and the olive oil placebo in that studies experienced some gastrointestinal problems (e.g. loose stools).

2. Fatigue, muscle soreness, muscle strains or pulls may result from the aerobic fitness and submaximal exercise testing.

3. The University will not be responsible for any medical expenses you may have unless the University has been negligent.

BENEFITS OF PARTICIPATION

Your participation will provide you with:

1. Data on your body composition and aerobic fitness.

COMPENSATION

We will pay you $\$ 50$ for full completion of the study.

\section{ANOYNMITY AND CONFIDENTIALITY}

The data from this study will be kept strictly confidential. No data will be released to anyone but those working on the project without your written permission. Data will be identified by subject numbers, without anything to identify subjects by name.

\section{FREEDOM TO WITHDRAW}

You are free to withdraw at any time from the study for any reason. Circumstances may come up that the researcher will determine that you should not continue as a subject in the study. For example, lack of compliance to diet or exercise, failure to attend testing sessions and illness could be reasons to have the researchers stop your participation in the study.

\section{APPROVAL OF RESEARCH}

This research has been approved, as required, by the Institutional Review Board for Research Involving Human Subjects at Virginia Tech, and by the Department of Human Nutrition, Foods, and Exercise. You will receive a copy of this form to take with you.

\section{SUBJECT PERMISSION}

I have read the informed consent and fully understand the procedures and conditions of the project. I have had all my questions answered, and I hereby give my voluntary consent to be a participant in this research study. I agree to abide by the rules of the project. I understand that I may withdraw from the study at any time. 
If I have questions, I will contact:

- Principal Investigator: Janet Walberg Rankin, Professor, Department of Human Nutrition, Foods, and Exercise. 231-6355

- Chairman, Institutional Review Board for Research Involving Human Subjects:David Moore, 231-4991

Name of Subject (please print)

Signature of Subject

Date 


\section{Informed Consent for Participants of Investigative Projects \\ Department of Human Nutrition, Foods, and Exercise Virginia Tech}

Title: The reliability and validity of the SenseWear Armband in the measurement of energy expenditure

Principal Investigators: Janet Walberg Rankin, Ph.D., Margaret Fruin

\section{Purpose:}

Many tools have been developed in an attempt to accurately estimate the number of calories someone is using during activity. Doubly labeled water, the gold standard in freeliving energy expenditure (EE) measurement, is too expensive and inaccessible; and physical activity records and recall questionnaires, pedometers, heart rate monitors, and uniaxial and triaxial accelerometers have been proven inaccurate. Some of these tools are accurate for walking on a level surface but are inaccurate when walking up a hill or riding on a cycle. Thus, new tools are needed to more accurately capture the energy cost of various activities in free-living subjects. A new tool, the SenseWear Armband (BodyMedia, Inc), has been developed but not highly tested in various exercise situations. The armband is designed to be worn throughout the day. It collects various information (see below) that later can be downloaded to estimate energy expenditure. The armband combines an array of multiple sensors including a 2-axis accelerometer, optional heart rate monitor, heat flux sensor, skin temperature thermistor, near-body ambient temperature sensor, and galvanic skin response sensor. The raw physiologic data obtained from each of these sensors is combined and used by proprietary algorithms to estimate EE in calories. The SenseWear Armband is promoted as providing an accurate estimation of free-living EE, although no studies have been published that determine the reliability or accuracy of this device. Before the SenseWear Armband can be tested in free-living conditions and eventually utilized publicly, laboratory tests must be performed to determine its validity in rest and exercise bouts compared with indirect calorimetry measurements of EE. Thus the purpose of this study is to determine the accuracy of the SenseWear Armband in measuring energy expenditure at rest and during steady-state walking on a treadmill with and without an inclination.

\section{General design}

You will come to the laboratory on only one occasion for approximately 1 hour and 45 minutes. We will do some baseline measurements that include your weight, height, and body fat percentage. Body fat percentage will be determined by skinfold measurement (measure the thickness of fat folds at three areas of your body) at the abdomen, chest, and thigh for the men and at the waist, thigh, and back of the arm for the women. Following the baseline testing, we will be comparing the energy expenditure measurement of the SenseWear Armband plus Polar heart rate monitor to that of indirect calorimetry, which involves you breathing through a mouthpiece connected to a computer analysis system that collects and analyzes your expired air. The SenseWear Armband is worn around your upper right arm, and the heart rate monitor is worn around your chest. You will first stand at rest for 10 minutes before exercise begins. Then, following a brief warm-up, you will walk on a treadmill for 10 minutes at $3 \mathrm{mph}, 10$ minutes at $4 \mathrm{mph}$, and 10 minutes at $4 \mathrm{mph}$ at a 5 
degree inclination. Upon the completion of exercise, you will once again stand at rest for 10 minutes. The armband and heart rate monitor will be worn and the mouthpiece with collect expired air for the entire exercise duration, as well as the resting periods before and after the exercise.

The indirect calorimetry mouthpiece is rubber and similar to a snorkel mouthpiece. The SenseWear Armband is smaller than a walkman (approximately 3.5"x3.0"x7.5") and is worn around the upper arm by use of an elastic band. The armband estimates energy expenditure by non-invasive sensors within and on the back of the armband; the sensors include an accelerometer to measure the motion of the body, a heat flux sensor, galvanic skin response sensor, and skin temperature thermistor. The Polar heart rate monitor is a small plastic strip that is worn around the chest next to the skin and also collects non-invasive data by sensing the heart beat.

\section{Summary of Subject Responsibilities}

1. Inform the experimenters prior to the study of any known medical conditions of which you are aware.

2. Refrain from consuming any food, tobacco, or caffeine or performing any vigorous exercise for 3 hours prior to your testing time.

\section{Risks of Participation}

The armband (SenseWear Armband, Bodymedia, Inc.) does not pose any risk to you. It is similar to wearing a heart rate monitor. The exercise is low intensity walking and low risk, but minimal risks are still possible (e.g. sprained ankle, twisted knee, or minor abrasion if one fell while on the treadmill).

\section{Benefits of Participation}

Your participation will provide you with:

1. An estimate of your body composition

2. The experience of participating in research while at Virginia Tech No other promise of benefit has been made.

\section{Anonymity and Confidentiality}

The data from this study will be kept strictly confidential. No data will be released to anyone but the principal investigator and graduate students involved in the project without your written permission. Data will be identified by subject number, without anything to identify subjects by name.

\section{Freedom to Withdraw}

You are free to withdraw at any time from the study for any reason. The researcher is also free to stop your participation in the study at any time if she/he believes that you have failed to comply with the requirements of the study or if you appear ill or in danger.

\section{Approval of Research}

This research has been approved, as required, by the Institutional Review Board for Research Involving Human Subjects at Virginia Tech and by the Department of Human Nutrition, Foods, and Exercise. You will receive a copy of this form to take with you. 


\section{Subject Permission}

I have read the informed consent and fully understand the procedures and conditions of the project. I have had all my questions answered, and I hereby give my voluntary consent to be a participant in this research study. I agree to abide by the rules of the project. I understand that I may withdraw from the study at any time.

If I have questions, I will contact:

- Principal Investigator: Janet Walberg Rankin, Professor, Department of Human Nutrition, Foods, and Exercise, 231-6355

- Chairman, Institutional Review Board for Research Involving Human Subjects: David Moore, 231-4991

Name of Subject (please print)

Signature of Subject

Date 


\section{VIRGINIA TECH LABORATORY FOR HEALTH AND EXERCISE SCIENCE MEDICAL AND HEALTH HISTORY}

Name:

Age:

Birth Date:

Address:

e-mail:

Phone Numbers: Home:

Work :

Person to Contact in Case of an Emergency:

Relationship:

Phone:

Primary Care Physician:

Phone:

Medical Insurance Carrier:

Are you employed by Virginia Tech?

Current Body Weight:

Height:

\section{MEDICAL HISTORY}

Please indicate any current or previous conditions or problems you have experienced or have been told by a physician you have had:

Heart disease or any heart problems:

Rheumatic Fever:

Respiratory disease or breathing problems (e.g. asthma):

Circulation problems:

Kidney disease or problems:

Urinary problems:

Musculoskeletal problems:

(i.e. Orthopedic injuries, osteoporosis)

Fainting and Dizziness:

High Cholesterol:

Diabetes:

Thyroid problems:

Mental illness:

Hypoglycemia (i.e. low blood sugar):

Epilepsy or seizures:

Blood clotting problems (e.g. hemophilia):

Liver disorders (e.g. hepatitis B):

Yes No
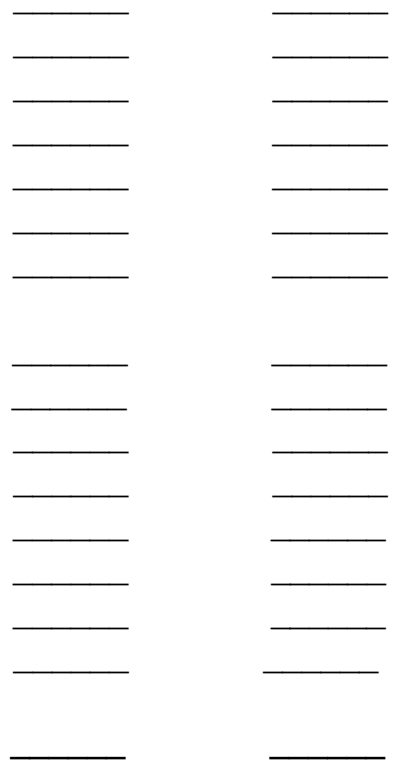

If you answered "yes" to any of the previous questions, please indicate the date and describe: 
Please list any hospitalizations/operations/recent illnesses (type/date):

$\begin{array}{lll}\text { Have you ever been diagnosed as having high blood pressure? } & \text { Yes } \\ \text { Are you currently being treated for high blood pressure? } & - \\ \text { If "yes", please explain: }\end{array}$

Please list all medications (prescription and over-the-counter) you are currently taking or have taken in the past week:

For what reason(s) are you taking this medication?

\section{Health Habits}

Do you drink alcoholic beverages?

How many drinks per week?

Do you smoke cigarettes?

Packs per day:

Do you engage in regular exercise?

If "yes", please list:

\section{Activity}

\begin{tabular}{ll} 
Yes & No \\
\hdashline$\quad$ & - \\
\hline Yes & No \\
\hline
\end{tabular}
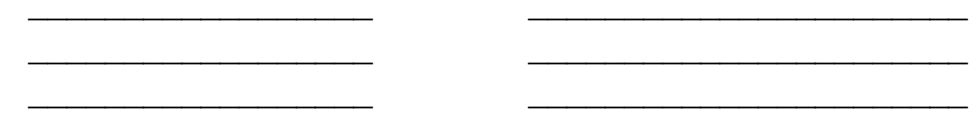

Duration (minutes)

Do you ever faint, experience shortness of breath or chest discomfort with exertion?

If "yes", please explain: 
Are there any orthopedic limitations you have that may restrict your ability to perform exercise and if "yes", please explain:

\section{Family History}

Has anyone in your family been diagnosed or treated for any of the following?

$\begin{array}{lllll}\text { Heart attack } & \text { Yes } & \text { No } & \text { Relationship } & \text { Age } \\ \text { Heart disease } & - & - & - & - \\ \begin{array}{l}\text { High blood pressure } \\ \text { Stroke }\end{array} & - & - & - & - \\ \text { Kidney disease } & - & - & - & - \\ \text { Diabetes } & - & - & -\end{array}$

Schedule Spring 2002 semester (indicate those times you have classes, work etc that you CANNOT be involved in testing or exercise training):

$$
\text { Monday Tuesday Wednesday Thursday Friday }
$$

6:00-7:00am

7:00-8:00

8:00-9:00

9:00-10:00

10:00-11:00

$11: 00-12: 00 \mathrm{pm}$

$12: 00-1: 00$

$1: 00-2: 00$

2:00-3:00

$3: 00-4: 00$

4:00-5:00

5:00-6:00

6:00-7:00

7:00-8:00

Any explanation required for above

Please sign to indicate that the above information is correct:

Print name

Signature

Date 


\section{Appendix F}

Data Record Sheet 
Subject:

Height: __ Weight:___ Age:

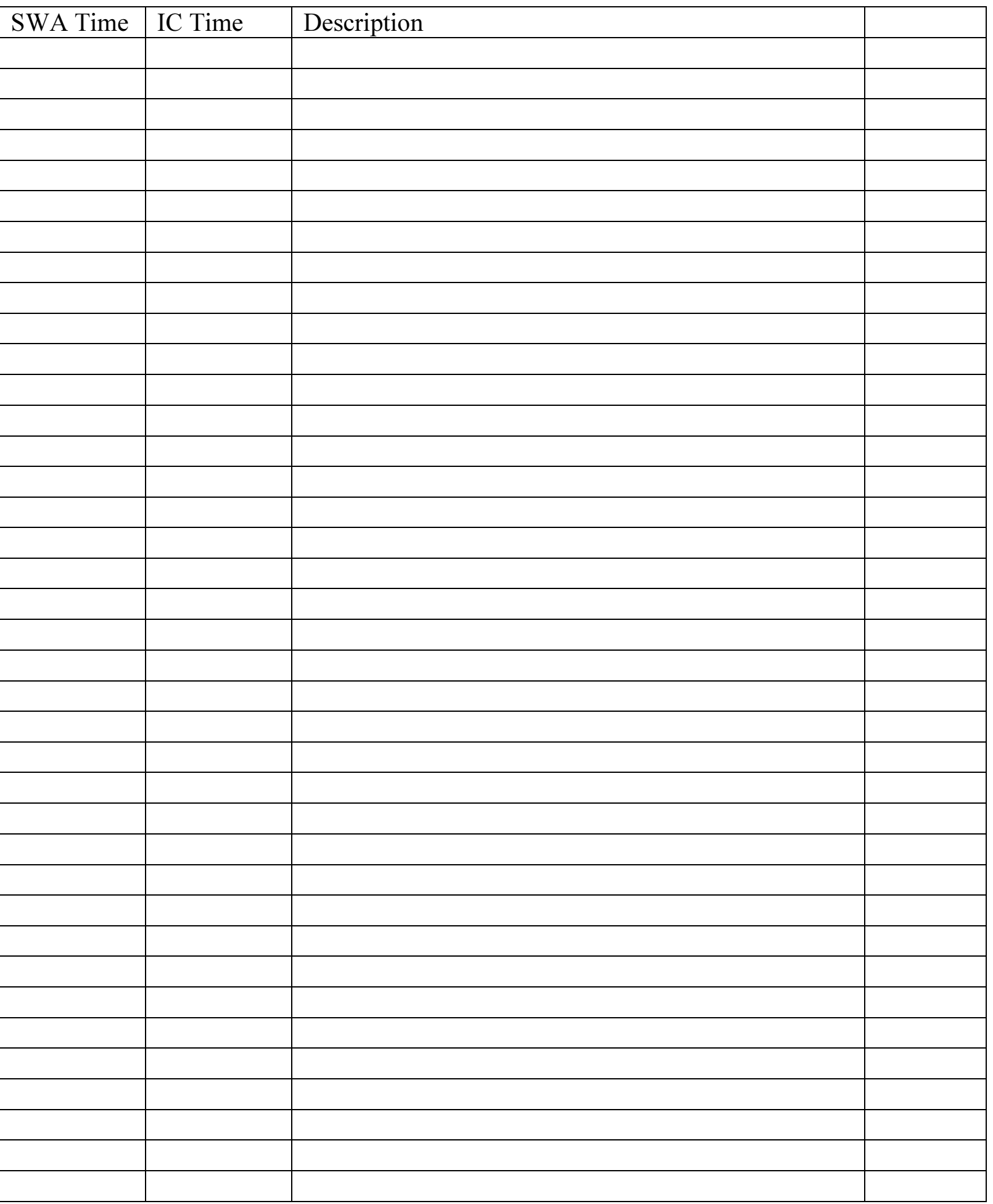




\section{REFERENCES}

1. Ainsworth, B. E., D. R. Bassett, Jr., S. J. Strath, A. M. Swartz, W. L. O'Brien, R. W. Thompson, D. A. Jones, C. A. Macera, and C. D. Kimsey. Comparison of three methods for measuring the time spent in physical activity. Med Sci Sports Exerc. 32:S457-464, 2000 .

2. Ainsworth, B. E., W. L. Haskell, A. S. Leon, D. R. Jacobs, Jr., H. J. Montoye, J. F. Sallis, and R. S. Paffenbarger, Jr. Compendium of physical activities: classification of energy costs of human physical activities. Med Sci Sports Exerc. 25:71-80, 1993.

3. Ainsworth, B. E., W. L. Haskell, M. C. Whitt, M. L. Irwin, A. M. Swartz, S. J. Strath, W. L. O'Brien, D. R. Bassett, Jr., K. H. Schmitz, P. O. Emplaincourt, D. R. Jacobs, Jr., and A. S. Leon. Compendium of physical activities: an update of activity codes and MET intensities. Med Sci Sports Exerc. 32:S498-504, 2000.

4. Balogun, J. A., D. A. Martin, and M. A. Clendenin. Calorimetric validation of the Caltrac accelerometer during level walking. Phys Ther. 69:501-509, 1989.

5. Bassett, D. R., Jr., B. E. Ainsworth, A. M. Swartz, S. J. Strath, W. L. O'Brien, and G. A. King. Validity of four motion sensors in measuring moderate intensity physical activity. Med Sci Sports Exerc. 32:S471-480, 2000.

6. Bland, J. M. and D. G. Altman. Statistical methods for assessing agreement between two methods of clinical measurement. Lancet. i:307-310, 1986.

7. BodyMedia Inc. SenseWear Armband Product Features. Available at: http://www.bodymedia.com/products/swpro.jsp Accessed January 19, 2003.

8. Bonnefoy, M., S. Normand, C. Pachiaudi, J. R. Lacour, M. Laville, and T. Kostka. Simultaneous validation of ten physical activity questionnaires in older men: a doubly labeled water study. J Am Geriatr Soc. 49:28-35, 2001.

9. Booth, F. W., M. V. Chakravarthy, S. E. Gordon, and E. E. Spangenburg. Waging war on physical inactivity: using modern molecular ammunition against an ancient enemy. $J$ Appl Physiol. 93:3-30, 2002.

10. Bouchard, C. and R. J. Shephard. Physical activity, fitness, and health : international proceedings and consensus statement. Champaign, IL: Human Kinetics Publishers, 1994, xxiv, 1055. 
11. Bratteby, L. E., B. Sandhagen, H. Fan, and G. Samuelson. A 7-day activity diary for assessment of daily energy expenditure validated by the doubly labelled water method in adolescents. Eur J Clin Nutr. 51:585-591, 1997.

12. Buchowski, M. S., K. M. Townsend, K. Y. Chen, S. A. Acra, and M. Sun. Energy expenditure determined by self-reported physical activity is related to body fatness. Obes Res. 7:23-33, 1999.

13. Campbell, K. L., P. R. Crocker, and D. C. McKenzie. Field evaluation of energy expenditure in women using Tritrac accelerometers. Med Sci Sports Exerc. 34:1667$1674,2002$.

14. Chasan-Taber, L., J. B. Erickson, P. C. Nasca, S. Chasan-Taber, and P. S. Freedson. Validity and reproducibility of a physical activity questionnaire in women. $\mathrm{Med} \mathrm{Sci}$ Sports Exerc. 34:987-992, 2002.

15. Chen, K. Y. and M. Sun. Improving energy expenditure estimation by using a triaxial accelerometer. J Appl Physiol. 83:2112-2122, 1997.

16. Close, W. H., M. J. Dauncey, and D. L. Ingram. Heat loss from humans measured with a direct calorimeter and heat-flow meters. Br J Nutr. 43:87-93, 1980.

17. Conway, J. M., J. L. Seale, D. R. Jacobs, Jr., M. L. Irwin, and B. E. Ainsworth. Comparison of energy expenditure estimates from doubly labeled water, a physical activity questionnaire, and physical activity records. Am J Clin Nutr. 75:519-525, 2002.

18. Dauncey, M. J. Influence of mild cold on 24 h energy expenditure, resting metabolism and diet-induced thermogenesis. Br J Nutr. 45:257-267, 1981.

19. Duncan, G. E., S. J. Sydeman, M. G. Perri, M. C. Limacher, and A. D. Martin. Can sedentary adults accurately recall the intensity of their physical activity? Prev Med. $33: 18-26,2001$.

20. Ekelund, U., A. Yngve, K. Westerterp, and M. Sjostrom. Energy expenditure assessed by heart rate and doubly labeled water in young athletes. Med Sci Sports Exerc. 34:1360$1366,2002$.

21. Flegal, K. M., M. D. Carroll, C. L. Ogden, and C. L. Johnson. Prevalence and trends in obesity among US adults, 1999-2000. Jama. 288:1723-1727, 2002.

22. Freedson, P. S., E. Melanson, and J. Sirard. Calibration of the Computer Science and Applications, Inc. accelerometer. Med Sci Sports Exerc. 30:777-781, 1998. 
23. Gonzalez-Alonso, J., B. Quistorff, P. Krustrup, J. Bangsbo, and B. Saltin. Heat production in human skeletal muscle at the onset of intense dynamic exercise. $J$ Physiol. 524 Pt 2:603-615, 2000.

24. Haskell, W. L., M. C. Yee, A. Evans, and P. J. Irby. Simultaneous measurement of heart rate and body motion to quantitate physical activity. Med Sci Sports Exerc. 25:109-115, 1993.

25. Hausswirth, C., A. X. Bigard, and J. M. Le Chevalier. The Cosmed K4 telemetry system as an accurate device for oxygen uptake measurements during exercise. Int J Sports Med. 18:449-453, 1997.

26. Haymes, E. M. and W. C. Byrnes. Walking and running energy expenditure estimated by Caltrac and indirect calorimetry. Med Sci Sports Exerc. 25:1365-1369, 1993.

27. Hendelman, D., K. Miller, C. Baggett, E. Debold, and P. Freedson. Validity of accelerometry for the assessment of moderate intensity physical activity in the field. Med Sci Sports Exerc. 32:S442-449, 2000.

28. Irwin, M. L., B. E. Ainsworth, and J. M. Conway. Estimation of energy expenditure from physical activity measures: determinants of accuracy. Obes Res. 9:517-525, 2001.

29. Jackson, A. S. and M. L. Pollock. Generalized equations for predicting body density of men. Br J Nutr. 40:497-504, 1978.

30. Jackson, A. S., M. L. Pollock, and A. Ward. Generalized equations for predicting body density of women. Med Sci Sports Exerc. 12:175-181, 1980.

31. Jacobs, D. R., Jr., B. E. Ainsworth, T. J. Hartman, and A. S. Leon. A simultaneous evaluation of 10 commonly used physical activity questionnaires. Med Sci Sports Exerc. 25:81-91, 1993.

32. Jakicic, J. M., K. Clark, E. Coleman, J. E. Donnelly, J. Foreyt, E. Melanson, J. Volek, and S. L. Volpe. ACSM position stand on the appropriate intervention strategies for weight loss and prevention of weight regain for adults. Med Sci Sports Exerc. 33:21452156, 2001.

33. Jakicic, J. M., B. A. Polley, and R. R. Wing. Accuracy of self-reported exercise and the relationship with weight loss in overweight women. Med Sci Sports Exerc. 30:634-638, 1998. 
34. Jakicic, J. M., C. Winters, K. Lagally, J. Ho, R. J. Robertson, and R. R. Wing. The accuracy of the TriTrac-R3D accelerometer to estimate energy expenditure. Med Sci Sports Exerc. 31:747-754, 1999.

35. Kasabach, C., C. Pacione, J. Stivoric, A. Teller, and D. Andre. Why the upper arm? Factors contributing to the design of an accurate comfortable, wearable body monitor. Available at: http://www.bodymedia.com/products/accuracy.jsp Accessed January 19, 2003.

36. King, G. A., J. E. McLaughlin, E. T. Howley, D. R. Bassett, Jr., and B. E. Ainsworth. Validation of Aerosport KB1-C portable metabolic system. Int J Sports Med. 20:304-308, 1999.

37. Kriska, A. M. and C.J. Caspersen. Introduction to a collection of physical activity questionnaires. Med Sci Sports Exerc. 29:S5-S9, 1997.

38. Leenders, N. Y., W. M. Sherman, H. N. Nagaraja, and C. L. Kien. Evaluation of methods to assess physical activity in free-living conditions. Med Sci Sports Exerc. 33:1233-1240, 2001.

39. Leenders, N. Y., W.M. Sherman, and H. N. Nagaraja. Comparisons of four methods of estimating physical activity in adult women. Med Sci Sports Exerc. 32:1320-1326, 2000.

40. Levine, J. A., P. A. Baukol, and K. R. Westerterp. Validation of the Tracmor triaxial accelerometer system for walking. Med Sci Sports Exerc. 33:1593-1597, 2001.

41. Lichtman, S. W., K. Pisarska, E. R. Berman, M. Pestone, H. Dowling, E. Offenbacher, H. Weisel, S. Heshka, D. E. Matthews, and S. B. Heymsfield. Discrepancy between selfreported and actual caloric intake and exercise in obese subjects. $N$ Engl J Med. 327:1893-1898, 1992.

42. Liden C.B., Wolowicz M., Stivoric J., Teller A., Kasabach C., Vishnubhatla S., Pelletier R., Farringdon J., and S. Boehmke. Characterization and implications of the sensors incorporated into the SenseWear ${ }^{\mathrm{TM}}$ Armband for energy expenditure and activity detection. Available at: http://www.bodymedia.com/products/accuracy.jsp Accessed November, 2002.

43. Liden, C. B., M. Wolowicz, J. Stivoric, A. Teller, C. Kasabach, S. Vishnubhatla, R. Pelletier, J. Farringdon, and S. Boehmke. Characterization and implications of the sensors incorporated into the SenseWear ${ }^{\mathrm{TM}}$ Armband for energy expenditure and activity 
detection. Available at: http://www.bodymedia.com/products/accuracy.jsp Accessed November, 2002.

44. Liden, C. B., M. Wolowicz, J. Stivoric, A. Teller, S. Vishnubhatla, R. Pelletier, and J. Farringdon. Accuracy and reliability of the SenseWear ${ }^{\mathrm{TM}}$ Armband as an energy expenditure assessment device. Available at: http://www.bodymedia.com/products/accuracy.jsp Accessed January, 2002.

45. Livingstone, M. B., A. M. Prentice, W. A. Coward, S. M. Ceesay, J. J. Strain, P. G. McKenna, G. B. Nevin, M. E. Barker, and R. J. Hickey. Simultaneous measurement of free-living energy expenditure by the doubly labeled water method and heart-rate monitoring. Am J Clin Nutr. 52:59-65, 1990.

46. Luke, A., K. C. Maki, N. Barkey, R. Cooper, and D. McGee. Simultaneous monitoring of heart rate and motion to assess energy expenditure. Med Sci Sports Exerc. 29:144-148, 1997.

47. Manore, M. and J. Thompson. Sport Nutrition for Health and Performance. Champaign, IL: Human Kinetics, 2000

48. McArdle, W. D., F. I. Katch, and V. L. Katch. Exercise physiology : energy, nutrition, and human performance. Philadelphia: Lea \& Febiger, 1981, xi, 508.

49. McKechnie, R. and L. Mosca. Physical activity and coronary heart disease: prevention and effect on risk factors. Cardiol Rev. 11:21-25, 2003.

50. McLaughlin, J. E., G. A. King, E. T. Howley, D. R. Bassett, Jr., and B. E. Ainsworth. Validation of the COSMED K4 b2 portable metabolic system. Int J Sports Med. 22:280284, 2001.

51. Meijer, G. A., K. R. Westerterp, H. Koper, and F. ten Hoor. Assessment of energy expenditure by recording heart rate and body acceleration. Med Sci Sports Exerc. 21:343347, 1989.

52. Montoye, H. J. Introduction: evaluation of some measurements of physical activity and energy expenditure. Med Sci Sports Exerc. 32:S439-441, 2000.

53. Montoye, H. J., H. C. G. Kemper, W. H. Saris, and R. A. Washburn. Measuring Physical Activity and Energy Expenditure. Champaign, IL: Human Kinetics, 1996 
54. Nichols, J. F., C. G. Morgan, J. A. Sarkin, J. F. Sallis, and K. J. Calfas. Validity, reliability, and calibration of the Tritrac accelerometer as a measure of physical activity. Med Sci Sports Exerc. 31:908-912, 1999.

55. Pate, R. R., M. Pratt, S. N. Blair, W. L. Haskell, C. A. Macera, C. Bouchard, D. Buchner, W. Ettinger, G. W. Heath, A. C. King, and et al. Physical activity and public health. A recommendation from the Centers for Disease Control and Prevention and the American College of Sports Medicine. Jama. 273:402-407, 1995.

56. Pearson, E. S. and H. O. Hartley. Charts of the power function for analysis of variance tests, derived from the non-central F-distribution. Biometrika. 38:112-130, 1951.

57. Powers, S. K. and E. T. Howley. Exercise physiology: theory and application to fitness and performance. fourth ed. New York, NY: McGraw-Hill Higher Education, 2001

58. Rennie, K., T. Rowsell, S. A. Jebb, D. Holburn, and N. J. Wareham. A combined heart rate and movement sensor: proof of concept and preliminary testing study. Eur J Clin Nutr. 54:409-414, 2000.

59. Richardson, M. T., B. E. Ainsworth, D. R. Jacobs, and A. S. Leon. Validation of the Stanford 7-day recall to assess habitual physical activity. Ann Epidemiol. 11:145-153, 2001.

60. Sallis, J. F. and B. E. Saelens. Assessment of physical activity by self-report: status, limitations, and future directions. Res Q Exerc Sport. 71:S1-14, 2000.

61. Saris, W. H. Fit, fat and fat free: the metabolic aspects of weight control. Int J Obes Relat Metab Disord. 22 Suppl 2:S15-21, 1998.

62. Seale, J. L., W. V. Rumpler, J. M. Conway, and C. W. Miles. Comparison of doubly labeled water, intake-balance, and direct- and indirect-calorimetry methods for measuring energy expenditure in adult men. Am J Clin Nutr. 52:66-71, 1990.

63. Sherman, W. M., D. M. Morris, T. E. Kirby, R. A. Petosa, B. A. Smith, D. J. Frid, and N. Leenders. Evaluation of a commercial accelerometer (Tritrac-R3 D) to measure energy expenditure during ambulation. Int J Sports Med. 19:43-47, 1998.

64. Starling, R. D., D. E. Matthews, P. A. Ades, and E. T. Poehlman. Assessment of physical activity in older individuals: a doubly labeled water study. $J$ Appl Physiol. 86:2090-2096, 1999. 
65. Strath, S. J., D. R. Bassett, Jr., A. M. Swartz, and D. L. Thompson. Simultaneous heart rate-motion sensor technique to estimate energy expenditure. Med Sci Sports Exerc. $33: 2118-2123,2001$.

66. Strath, S. J., D. R. Bassett, Jr., D. L. Thompson, and A. M. Swartz. Validity of the simultaneous heart rate-motion sensor technique for measuring energy expenditure. Med Sci Sports Exerc. 34:888-894, 2002.

67. Strath, S. J., A. M. Swartz, D. R. Bassett, Jr., W. L. O'Brien, G. A. King, and B. E. Ainsworth. Evaluation of heart rate as a method for assessing moderate intensity physical activity. Med Sci Sports Exerc. 32:S465-470, 2000.

68. Swartz, A. M., S. J. Strath, D. R. Bassett, Jr., W. L. O'Brien, G. A. King, and B. E. Ainsworth. Estimation of energy expenditure using CSA accelerometers at hip and wrist sites. Med Sci Sports Exerc. 32:S450-456, 2000.

69. Tudor-Locke, C., B. E. Ainsworth, R. W. Thompson, and C. E. Matthews. Comparison of pedometer and accelerometer measures of free-living physical activity. Med Sci Sports Exerc. 34:2045-2051, 2002.

70. Tudor-Locke, C., J. E. Williams, J. P. Reis, and D. Pluto. Utility of pedometers for assessing physical activity: convergent validity. Sports Med. 32:795-808, 2002.

71. U.S. Department of Health and Human Services. Healthy People 2010: Understanding and Improving Health. 2nd ed. Washington, DC: U.S. Government Printing Office, November 2000.

72. U.S. Department of Health and Human Services. Physical Activity and Health: A Report of the Surgeon General. Atlanta, GA: U.S. Department of Health and Human Services, Centers for Disease Control and Prevention, National Center for Chronic Disease Prevention and Health Promotion, 1996, pp. 29-37.

73. Valencia, M. E., G. McNeill, J. M. Brockway, and J. S. Smith. The effect of environmental temperature and humidity on 24 h energy expenditure in men. Br J Nutr. 68:319-327, 1992.

74. Welk, G. J., S. N. Blair, K. Wood, S. Jones, and R. W. Thompson. A comparative evaluation of three accelerometry-based physical activity monitors. Med Sci Sports Exerc. 32:S489-497, 2000. 
75. Welk, G. J. and C. B. Corbin. The validity of the Tritrac-R3D Activity Monitor for the assessment of physical activity in children. Res Q Exerc Sport. 66:202-209, 1995.

76. Welk, G. J., C. B. Corbin, and J. B. Kampert. The validity of the Tritrac-R3D activity monitor for the assessment of physical activity: II. Temporal relationships among objective assessments. Res Q Exerc Sport. 69:395-399, 1998.

77. Welk, G. J., J. A. Differding, R. W. Thompson, S. N. Blair, J. Dziura, and P. Hart. The utility of the Digi-walker step counter to assess daily physical activity patterns. Med Sci Sports Exerc. 32:S481-488, 2000.

78. Westerterp, K. R. Assessment of physical activity level in relation to obesity: current evidence and research issues. Med Sci Sports Exerc. 31:S522-525, 1999.

79. Westerterp, K. R. Physical activity assessment with accelerometers. Int J Obes Relat Metab Disord. 23 Suppl 3:S45-49, 1999.

80. Yngve, A., A. Nilsson, M. Sjostrom, and U. Ekelund. Effect of monitor placement and of activity setting on the MTI accelerometer output. Med Sci Sports Exerc. 35:320-326, 2003. 


\section{VITA}

Margaret Louise Fruin was born on June 6, 1978 in Marshalltown, Iowa. She graduated Summa Cum Laude from Kansas State University in 2001 with Bachelor of Science degrees in Human Nutrition and Kinesiology. She is pursuing a Master of Science degree in Human Nutrition, Foods, and Exercise from Virginia Tech with the option of Nutrition In Sports and Chronic Disease. Upon completion of the degree requirements, she will be completing a dietetic internship at Vanderbilt University Medical Center in Nashville, Tennessee. 